\title{
Esterase inhibition by grapefruit juice and its components leads to newly identified drug interactions
}

Ping Li

West Virginia University

Follow this and additional works at: https://researchrepository.wvu.edu/etd

\section{Recommended Citation}

Li, Ping, "Esterase inhibition by grapefruit juice and its components leads to newly identified drug interactions" (2006). Graduate Theses, Dissertations, and Problem Reports. 2446.

https://researchrepository.wvu.edu/etd/2446

This Dissertation is protected by copyright and/or related rights. It has been brought to you by the The Research Repository @ WVU with permission from the rights-holder(s). You are free to use this Dissertation in any way that is permitted by the copyright and related rights legislation that applies to your use. For other uses you must obtain permission from the rights-holder(s) directly, unless additional rights are indicated by a Creative Commons license in the record and/ or on the work itself. This Dissertation has been accepted for inclusion in WVU Graduate Theses, Dissertations, and Problem Reports collection by an authorized administrator of The Research Repository @ WVU.

For more information, please contact researchrepository@mail.wvu.edu. 


\title{
Esterase inhibition by grapefruit juice and its components leads to newly Identified drug interactions
}

\section{Ping Li}

\author{
Dissertation submitted to the \\ School Pharmacy at West Virginia University \\ in partial fulfillment of the requirements for the degree of \\ Doctor of Philosophy
in
Pharmaceutical Sciences
}

Suresh K. Balani, Ph.D.

Peter M. Gannett, Ph.D.

Robert L. Haining, Ph.D.

Grazyna D. Szklarz, Ph.D.

Patrick S. Callery, Ph.D., Chair

Morgantown, West Virginia University

2006

Keywords: Grapefruit juice, Esterases, Hydrolysis, Inhibition, Prodrugs, Lovastatin, Enalapril, Pharmacokinetics 


\section{ABSTRACT \\ Esterase inhibition by grapefruit juice and its components leads to newly Identified drug interactions \\ Ping Li}

Grapefruit juice (GFJ) is well known to be a potent inhibitor of the CYP3A enzyme leading to drug-drug interactions. This study describes a newly identified potential of GFJ in mediating pharmacokinetic drug-drug interactions due to its capability of esterase inhibition. The study demonstrates that GFJ inhibits purified porcine esterase activity towards para-nitrophenylacetate (PNPA) and the prodrugs lovastatin and enalapril. In rat and human hepatic or gut S9 fractions and rat gut lumen GFJ inhibited the hydrolysis of enalapril and lovastatin, which are known to be metabolized principally by esterases, with lovastatin metabolized also by CYP3A. In Caco-2 cells, permeability of these prodrugs was increased in the presence of GFJ. In rats, oral coadministration of GFJ or an esterase inhibitor bis-(p-nitrophenylphosphate) with the prodrugs led to respective increases in plasma AUC for enalaprilat for lovastatin acid. Studies in portal vein cannulated rats demonstrated that in addition to CYP3A inactivation, GFJ also played a significant role in the decreased esterase activity leading to enhancement of exposure to the active drugs in rats.

To identify the GFJ components responsible for this new GFJ-prodrug interaction, the esterase inhibitory potential of ten constituitive flavonoids and furanocoumarins was investigated. The furanocoumarins bergamottin, 6', 7'dihydroxybergamottin and bergapten and the glycosidic flavonoids naringin and hesperidin, at concentrations found in GFJ or higher did not inhibit the hydrolysis of PNPA by purified porcine esterase and human liver microsomes. However, the flavonoid aglycones morin, galangin, kaempferol, quercetin, and naringenin showed appreciable inhibition of PNPA hydrolysis. In Caco-2 cells, the permeability coefficient of prodrug lovastatin and enalapril was increased in the presence of the active flavonoids kaempferol and naringenin, consistent with inhibition of esterase activity. In rats, oral coadministration of kaempferol and naringenin with these prodrugs led to significant increases in plasma exposure to the active acids. Overall, a series of flavonoids present in GFJ are identified as carboxylesterase inhibitors, of which kaempferol, and naringenin are shown to mediate pharmacokinetic drug interaction with prodrugs lovastatin and enalapril due to their ability to inhibit esterase. 


\section{ACKNOWLEDGEMENTS}

I am very grateful to Dr. Patrick S. Callery, who believed in me all the way. His support, encouragement, and guidance have made the impossible possible.

I would like to express my sincere appreciation to Dr. Suresh K. Balani for his excellent guidance and valuable time. His commitment was the key for the success of my Ph.D. project.

I also want to thank my graduate committee Drs. Liang-Shang Gan, Peter Gannett, Robert L. Haining, and Grazyna D. Szklarz for their invaluable suggestions.

I would like to thank Millennium Pharmaceuticals Inc. and my colleagues

at Millennium especially Kym Cardoza, Ning Liu, Cindy Xia, Chuang Lu, LiangShang Gan, and Gerald Miwa for their support.

I would like to dedicate this work to my son Michael and my husband Zhongguo. There are no words to express my deep gratitude to them. Their unconditioned love and support help me fulfill this lifelong dream. 


\section{INTRODUCTION}

Since the first report of the grapefruit juice (GFJ) effect on the oral bioavailability of felodipine (Bailey et al., 1989, 1991), GFJ has been shown to increase oral exposure of many compounds (Kupferschmidt et al., 1995, Benton et al., 1996, Ducharme et al., 1995). These drugs differ in their chemical and pharmacological properties, but are, in common, extensively metabolized by CYP3A. The mechanism of action was postulated to be competitive and mechanism-based inhibition of CYP3A4/5 (hereafter referred to as CYP3A) in the small intestine by GFJ (Schmiedlin-Ren et al. 1997; He et al., 1998). The effect is believed to be at the level of intestine because GFJ showed little effect after single intravenous administration (Kupferschmidt et al., 1995; Ducharme et al., 1995). Although some recent reports point to the inhibitory effects of grapefruit juice on the function of P-glycoprotein (Zhou et al., 2004) and Organic Anion Transporting polypeptides (OATP) (Dresser et al., 2002), the contribution to the bioavailability of drugs that are substrates of P-glycoprotein and OATP has not been well established. Since 1998, the effect of grapefruit juice ingestion on the pharmacokinetics of orally administered drugs have been reported for 40 drugs (Saito et al., 2005), generally related to CYP3A inhibition. Effect of repeated intake of GFJ on CYP3A activity (Culm-merdek et al, 2006) has been reported. It is interesting to note that the magnitude of GFJ effect varied greatly, and we noted that the magnitude of GFJ effect was not proportional to the extent of CYP3A-mediated intestinal metabolism. As an example, cyclosporine, which is extensively metabolized in human intestine by CYP3A, interacted weakly with GFJ (Ducharme et al., 1995), the interaction unlikely to be of clinical significance. However, lovastatin which is also metabolized in human intestine by CYP3A and esterase, led to one of the most potent and clinically significant drug-GFJ interactions. The oral bioavailability of lovastatin in humans was increased $>15$ fold by GFJ (Kantola et al., 1998). Because lovastatin is a CYP3A substrate, this interaction was postulated to be the result of CYP3A inhibition. However, since

lovastatin (a lactone) is also known to be hydrolyzed by esterase to a 
hydroxyacid analog (active drug), and the carboxylesterase (CE) mediated hydrolysis of lovastatin is also a major metabolic pathway (Halpin et al., 1993), we hypothesized that the interaction between GFJ and lovastatin is cumulative of CYP3A and esterase inhibition. There are a few reports on the interactions between esterase inhibitors and ester compounds, including naturally occurring products. Extract of strawberry and banana juice inhibited the intestinal esterase-mediated hydrolysis of antiviral ester prodrug bis (POC)-PMPA (VanGelder et al., 1999) and increased absorption of the ester prodrug tenofovir disoproxil in rat ileum by inhibiting its intestinal metabolism (VanGelder et al., 2000). Intestinal absorption of tenofovir disoproxil fumarate was enhanced also by a defined esters mixture (Van Gelder et al., 2002). However, there are no reports on GFJ mediated interactions due b esterase inhibition. We designed and performed these studies, described in three separate sections, to test our hypothesis that GFJ inhibits esterases. In Part I, carboxyl esters lovastatin and enalapril were selected as model compounds to investigate the GFJ-carboxyl ester interactions. In vitro, evaluation of the esterase-mediated changes in the permeability and metabolism in in vitro systems, and in vivo rat exposure to enalapril and lovastatin when coadministered with GFJ are described. In Part II, esterase inhibition potential of ten grapefruit juice components towards PNPA hydrolysis as well as the effect of two selected carboxyl esters on the esterase mediated changes in the permeability in in vitro systems, and in in vivo rat exposure to active acids of enalapril and lovastatin upon coadministration are described. In Part III, the interaction mechanism of grapefruit components with enalapril and CPT11, two selective substrates for human carboxylesrease I and II, respectively, were further studied. Every experiment in this dissertation was performed in Millennium Pharmaceuticals Inc. (Cambridge, MA), and all animal in-life studies were performed by Comparative Medicine Group. 


\section{TABLE OF CONTENTS}

\section{Page}

ACKNOWLEDGMENTS

iii

TABLE OF CONTENTS

vi

LIST OF TABLES $\quad$ ix

LIST OF FIGURES X xii

INDRODUCTION iv

$\begin{array}{ll}\text { SUMMARY } & 44\end{array}$

\section{PART I}

Esterase inhibition by grapefruit juice leads to a newly identified drug interaction

1. INTRODUCTION 2

2. OBJECTIVES 3

3. MATERIALS AND METHODS 3

3.1 Materials 4

3.2 Effect of GFJ on purified porcine esterases 4

3.3 Effect of GFJ on hydrolysis of enalapril and lovastatin in 5 human and rat in vitro system

3.4 Effect of GFJ on A-to-B permeability of lovastatin and 6 enalapril across Caco-2 membrane

3.5 Pharmacokinetic studies in SD rats 9

RESULTS AND DISCUSSION 
5. CONCUSIONS 20

$\begin{array}{ll}\text { 6. FIGURES } & 69\end{array}$

7. TABLES 54

8. BIBLIOGRAPHY 46

\section{PART II}

Esterase inhibition by grapefruit juice components leads to a newly identified drug interaction

9. INTRODUCTION

10. OBJECTIVES

11. MATERIALS AND METHODS 24

$\begin{array}{ll}11.1 \text { Materials } & 24\end{array}$

11.2 Effect of GFJ chemical constituents on purified porcine esterases 25

11.3 Effect of GFJ chemical constituents on esterases in human 26 liver microsmes

11.4 Effect of GFJ chemical constituents on A-to-B permeability 26 of lovastatin and enalapril across Caco-2 membrane

11.5 Pharmacokinetic studies in SD rats 27

12. RESULTS AND DISCUSSION 29

13. CONCLUSIONS 34

$\begin{array}{ll}\text { 6. FIGURES } & 81\end{array}$

$\begin{array}{ll}\text { 7. TABLES } & 61\end{array}$

8. BIBLIOGRAPHY 46 


\section{PART III}

The interaction mechanism of grapefruit juice chemical components with esterases

$\begin{array}{ll}\text { 14. INTRODUCTION } & 37\end{array}$

15. OBJECTIVES 38

16. MATERIALS AND METHODS 38

16.1 Hydrolysis of CPT-11 38

16.2 Hydrolysis of enalapril $\quad 39$

16.3 Michaelis-Menten kinetics of CPT-11 hydrolysis by purified $\quad 40$ porcine esterases, and the effect of GFJ chemical constituents

16.4 Michaelis-Menten kinetics of enalapril hydrolysis by purified porcine esterases, and the effect of GFJ chemical constituents

17. RESULTS AND DISCUSSION 42

18. CONCLUSIONS 44

$\begin{array}{ll}\text { 6. FIGURES } & 95\end{array}$

$\begin{array}{lll}\text { 7. TABLES } & 66\end{array}$

8. BIBLIOGRAPHY 46 


\section{LIST OF TABLES}

Table1. Percentage remaining of lovastatin in pulp-free GFJ

supernatant after the centrifugation of lovastatin $(20 \mu \mathrm{M})$

solutions containing various concentrations of GFJ

Table2. Effect of the esterase inhibitor BNPP (25 mg/kg PO) on

the PK parameters of lovastatin acid and enalaprilat

following oral administration of lovastatin and enalapril, respectively, $(10 \mathrm{mg} / \mathrm{kg})$ to rats

Table3. Effect of GFJ (10 mL/kg) on PK parameters of lovastatin acid and enalaprilat following oral administration of lovastatin $(10 \mathrm{mg} / \mathrm{kg})$ and enalapril $(10 \mathrm{mg} / \mathrm{kg})$ to rats

Table4. Portal plasma PK parameters of lovastatin acid and lovastatin

+ lovastatin acid following oral coadministration of lovastatin (10 $\mathrm{mg} / \mathrm{kg}$ ) with water or GFJ to portal vein-cannulated rats, pretreated at -15 and $-2 \mathrm{hr}$ with acidified water or GFJ

Table5. Portal plasma PK parameters of 6'B-hydroxylovastatin and its hydroxy acid (6'ß-hydroxylovastatin acid) following oral administration of lovastatin $(10 \mathrm{mg} / \mathrm{kg})$ with water $(\mathrm{pH} \mathrm{3.5)}$ or GFJ to portal vein-cannulated rats, pretreated at -15 and $-2 \mathrm{hr}$ with water or GFJ

Table6. Effect of oral pretreatment with GFJ on PK parameters of lovastatin and lovastatin acid following IV administration of 
lovastatin $(2 \mathrm{mg} / \mathrm{kg})$ to rats

Table7. Effect of oral pretreatment with GFJ on PK parameters of 60 enalarpil and enalaprilat following IV administration of enalapril $(2 \mathrm{mg} / \mathrm{kg})$ to rats

Table8. Inhibitory effect of GFJ chemical components toward PNPA hydrolysis in purified porcine esterases

Table9. Inhibitory effect of GFJ chemical components toward PNPA hydrolysis in human liver microsomes

Table10. Pharmacokinetic parameters of enalaprilat following oral administration of enalapril $(10 \mathrm{mg} / \mathrm{kg})$ to rats and the effect of co-administrated naringenin ( 2 and $10 \mathrm{mg} / \mathrm{kg}$ ) and kaempferol (2 and $10 \mathrm{mg} / \mathrm{kg}$ )

Table11. Pharmacokinetic parameters of lovastatin acid following oral administration of lovastatin $(10 \mathrm{mg} / \mathrm{kg})$ to rats and the effect of co-administrated naringenin ( 2 and $10 \mathrm{mg} / \mathrm{kg}$ ) and kaempferol (2 and $10 \mathrm{mg} / \mathrm{kg}$ )

Table12. Pharmacokinetic parameters of lovastatin, lovastatin acid, and 6'B-hydroxylovastatin in portal vein following oral administration of lovastatin $(10 \mathrm{mg} / \mathrm{kg})$ with water and kaempferol,10 mg/kg

Table 13. Parameters of Michaelis-Menten kinetics of CPT11 hydrolysis 66 by purified porcine esterases, and the effect of naringenin kaempferol, and quercetin 
Table14. Parameters of Michaelis-Menten kinetics of enalapril by

purified porcine esterases, and the effect of naringenin

kaempferol, and quercetin

Table15. Inhibition constants of kaempferol, quercetin,

68

naringenin on CPT11 and enalapril hydrolysis by

purified porcine esterases 


\section{LIST of FIGURES}

Figure.1 PNPA hydrolysis by purified porcine esterases

69

Figure.2 Inhibition of purified porcine esterases by GFJ 70

Figure.3 Inhibition of hydrolysis of (A) lovastatin $(5 \mu \mathrm{M})$ and $(B)$ enalapril $(5 \mu \mathrm{M})$ in human intestinal and liver S9 fraction

Figure.4 Inhibition of hydrolysis of lovastatin $(5 \mu \mathrm{M})$ in $(A)$ rat 72 Intestinal and liver S9 (B) intestinal lumen by GFJ

Figure.5 Inhibition of hydrolysis of enalapril $(5 \mu \mathrm{M})$ in $(\mathrm{A})$ rat intestinal 73 and liver S9 (B) rat intestinal lumen by GFJ

Figure.6 The CYP3A activity of Caco-2 homogenates 74

Figure.7 Hydrolysis of lovastatin by Caco-2 homogenates 75

Figure.8 Effects of GFJ on (A) lovastatin A $\rightarrow$ B permeability across $\quad 76$

Caco-2 membrane (B) ratio of lovastatin acid and lovastatin in Caco-2 cells at 1 hour

Figure.9 Effects of GFJ on (A) enalapril $A \rightarrow B$ permeability across Caco-2 membrane $(\mathrm{B})$ ratio of enalaprilat and enalapril in Caco- 2 cells at 1 hour

Figure.10 The uptake rate of enalapril into (A) Caco-2 cells, and the effect of GFJ (B) human PEPT1-expressed Oocytes

Figure.11 Full scan mass chromatogram(150-600 m/z) of incubation 79 mixture of lovastatin in human liver microsomes w/o NADPH

Figure.12 Full scan mass chromatogram $(150-600 \mathrm{~m} / \mathrm{z})$ of incubation mixture of lovastatin in human liver microsomes w/ NADPH 
Figure.13 SIM scan ( $\mathrm{m} / \mathrm{z} 405.3)$ of incubation mixtures of lovastatin in human liver microsomes w/ NADPH

Figure.14 SIM scan (m/z 423.3) of incubation mixtures of lovastatin in human liver microsomes w/ NADPH

Figure.15 Single ion $(\mathrm{m} / \mathrm{z} 321.5)$ extracted from full scan $(150-600 \mathrm{~m} / \mathrm{z}) \quad 83$

Figure.16 SIM scan (m/z 421.3) of incubation mixtures of lovastatin 84 in human liver microsomes w/ NADPH

Figure.17 SIM scan (m/z 439.3) of incubation mixtures of lovastatin 85 in human liver microsomes w/ NADPH

Figure.18 MS/MS of lovastatin ( $\mathrm{m} / \mathrm{z}$ 405.3)

Figure.19 MS/MS of lovastatin acid (m/z 423.3)

Figure.20 MS/MS of hydroxylovastatin $\mathrm{m} / \mathrm{z} 421.3, \mathrm{t}_{\mathrm{R}} 5.02 \mathrm{~min}$

Figure.21 MS/MS of hydroxylovastatin $\mathrm{m} / \mathrm{z} 421.3, \mathrm{t}_{\mathrm{R}} 6.19 \mathrm{~min}$

Figure.22 MS/MS of hydroxylovastatin acid $\mathrm{m} / \mathrm{z} 439.3, \mathrm{t}_{\mathrm{R}} 4.36 \mathrm{~min}$

Figure.23 MS/MS of hydroxylovastatin acid m/z 439.3, $t_{R} 5.67 \mathrm{~min}$

Figure.24 Effect of the esterase inhibitor BNPP (25 mg/kg PO) on the plasma concentration-time profiles of $(A)$ enalaprilat and (B) lovastatin acid following oral administration of enalapril and lovatatin at $10 \mathrm{mg} / \mathrm{kg}$, respectively

Figure.25 Plasma concentration-time profiles of $(\mathrm{A})$ enalaprilat and (B) lovastatin acid following oral administration of enalapril and lovastatin at $10 \mathrm{mg} / \mathrm{kg}$ with water or GFJ (1:3.1:2, Concentrate), respectively 
Figure.26 Portal plasma concentration-time profiles of $(A)$

lovastatin acid and $(B)$ lovastatin acid plus lovastatin

following oral administration of lovastatin $(10 \mathrm{mg} / \mathrm{kg})$

with water ( $\mathrm{pH}$ 3.5) or GFJ to portal vein cannulated rats,

pretreated at -15 and $-2 \mathrm{hr}$ with water $(\mathrm{pH} 3.5)$ or GFJ

Figure.27 Portal plasma concentration-time profiles of (A) 6'B-hydroxy

95

lovastatin (B) 6'ß-hydroxylovastatin acid following oral

administration of lovastatin $(10 \mathrm{mg} / \mathrm{kg})$ with water $(\mathrm{pH} 3.5)$ or

GFJ to portal vein-cannulated rats, pretreated at -15 and

-2 hr with water ( $\mathrm{pH} 3.5)$ or GFJ

Figure.28 PNPA hydrolysis by human liver microsomes 96

Figure.29 Inhibition potential of purified porcine esterases by 97

(A) naringin and (B)hesperidin

Figure.30 Inhibition potential of purified porcine esterases by (A)

98

bergamottin (B) 6',7'-dihydroxybergamottin, and (C) bergapten

Figure.31 Inhibition potential of purified porcine esterases by

99

(A) kaempferol, (B) quercetin, and (C) naringenin

Figure.32 inhibition potential of purified porcine esterases by

(A) morin, (B) galangin, and (C) BNPP

Figure.33 Inhibition potential of esterases of human liver microsomes by

(A) naringin and $(B)$ hesperidin

Figure.34 Inhibition potential of esterases of human liver microsomes by 102 (A) bergamottin, (B) 6', 7'-DHB, and (C) bergapten 
Figure.35 Inhibition potential of esterases of human liver microsomes by

(A) kaempferol, (B) quercetin, and (C) naringenin

Figure.36 Inhibition potential of esterases of human liver microsomes by 104 (A) morin, (B) galangin, and (C) BNPP

Figure.37 Effect of grapefruit juice constituents on $(A)$ lovastatin $A \rightarrow B \quad 105$ permeability across Caco-2 membrane $(B)$ ratio of lovastatin acid and lovastatin in Caco-2 cells at $1 \mathrm{hr}$

Figure.38 Effect of grapefruit juice constituents on (A) enalapril $A \rightarrow B \quad 106$ permeability across Caco-2 membrane $(B)$ ratio of enalaprilat and enalapril in Caco-2 cells at $1 \mathrm{hr}$

Figure.39 Plasma concentration-time profiles of enalaprilat following oral administration of enalapril at $10 \mathrm{mg} / \mathrm{kg}$ with $(\mathrm{A})$ water or naringenin (2 and $10 \mathrm{mg} / \mathrm{kg}$ ) (B) water or kaempferol (2 and $10 \mathrm{mg} / \mathrm{kg}$ )

Figure.40 Plasma concentration-time profiles of lovastatin acid following 108 oral administration of lovastatin at $10 \mathrm{mg} / \mathrm{kg}$ with $(\mathrm{A})$ water or naringenin (2 and $10 \mathrm{mg} / \mathrm{kg}$ ) (B) water or kaempferol (2 and $10 \mathrm{mg} / \mathrm{kg}$ )

Figure.41 Portal plasma concentration-time profiles of (A) lovastatin acid (B) lovastatin, and (C) 6'B-hydroxylovastatin following oral administration of lovastatin $(10 \mathrm{mg} / \mathrm{kg})$ with water or kaempferol $(10 \mathrm{mg} / \mathrm{kg})$

Figure.42 Chemical hydrolysis of (A) CPT11 (B) enalapril in $0.1 \mathrm{M}$ 
phosphate buffer, $\mathrm{pH}, 4.6,7.0$, and 8.0

Figure.43 Michaelis-Menten kinetics of CPT11 hydrolysis in human liver microsomes and S9 fraction

Figure.44 Michaelis-Menten kinetics of enalapril hydrolysis in human liver microsomes and S9 fraction

Figure.45 Michaelis-Menten kinetics of CPT11 hydrolysis by purified porcine esterases, and the effect of kaempferol (A) Direct plot and (B) Primary Lineweaver-Burk plot

Figure.46 Michaelis-Menten kinetics of CPT11 hydrolysis by purified porcine esterases, and the effect of naringenin (A) Direct plot and (B) Primary Lineweaver-Burk plot

Figure.47 Michaelis-Menten kinetics of CPT11 hydrolysis by purified porcine esterases, and the effect of quercetin (A) Direct plot and (B) Primary Lineweaver-Burk plot

Figure.48 Michaelis-Menten kinetics of enalapril hydrolysis by purified porcine esterases, and the effect of kaempferol (A) Direct plot and (B) Eadie-Hofstee plot

Figure.49 Michaelis-Menten kinetics of enalapril hydrolysis by purified porcine esterases, and the effect of quercetin (A) Direct plot and (B) Eadie-Hofstee plot

Figure.50 Michaelis-Menten kinetics of enalapril hydrolysis by purified porcine esterases, and the effect of nargingenin (A) Direct plot and (B) Eadie-Hofstee plot 
Figure. 51 Metabolism of lovastatin in human liver microsomes 119

Figure. 52 Structures of studied carboxyl esters and corresponding 120 free acids

Figure. 53 Structures of studied GFJ chemical components 121

Figure. 54 Structures of PMSF, BNPP, Gly-Sar, and GF210918 122 


\section{PART I}

ESTERASE INHIBITION BY GRAPEFRUIT JUICE LEADS TO A NEWLY IDENTIFIED DRUG INTERACTION 


\section{INTRODUCTION}

In humans, carboxylesterases (CEs) metabolize a wide variety of esterified drugs such as meperidine (Zhang et.al, 1997) and cefuroximine axetil (Campbell et al., 1987), anticancer agents CPT11 (Potter et al., 1998; Danks et al., 1999) and capecitabine (Campbell et al., 1987), as well as the illicit street drugs heroin and cocaine (Pindel et al., 1997). In general, CEs are expressed in tissues likely to be exposed to such agents including the liver, small intestine, kidney, etc (Satoh T. and Hosokawa M., 1998). Therefore, agents affecting CEs could result in alterations in distribution and clearance of esterified drugs leading to potential CE-mediated drug interactions. There are a few reports on the interactions between esterase inhibitors and ester compounds, including naturally occurring products. Extract of strawberry and banana juice inhibited the intestinal esterase-mediated hydrolysis of antiviral ester prodrug bis (POC)-PMPA (VanGelder et al., 1999) and increased absorption of the ester prodrug tenofovir disoproxil in rat ileum by inhibiting its intestinal metabolism (VanGelder et al., 2000). Intestinal absorption of tenofovir disoproxil fumarate was enhanced also by a defined esters mixture (Van Gelder et al., 2002). However, there are no reports on GFJ mediated interactions due to esterase inhibition. In this study we used carboxyl esters lovastatin and enalapril as model compounds to investigate the GFJ-carboxyl ester interactions. Lovastatin, a prodrug for the treatment of hypercholesterolemia, is metabolized by CYP3A4 to oxidative products and hydrolyzed by carboxylesterase to its active form lovastatin hydroxyl acid (hereafter referred to as lovastatin acid) (Halpin et al., 1993). The hydrolysis occurs in gut, liver and plasma and is considered as its major metabolic pathway (Vree et al., 2003). Lovastatin absorption is about $30 \%$ in humans as well in rats (Duggan et al., 1988). The similarity of lovastatin absorption in rats and humans led us to employ the rat as a preclinical animal model to elucidate ester mediated alterations in systemic exposure in rats upon coadministration with GFJ. 
The second compound selected for the current studies was enalapril, a prodrug for the treatment of hypertension. Enalaprilat, the hydroxyacid form of this alkyl ester prodrug, is a potent inhibitor of diacidic angiotensin converting enzyme (ACE). The bioavailability of enalaprilat in humans (Hockings et al., 1986) is increased to $50-60 \%$ when given as enalapril, compared to only $3 \%$ when enalaprilat is administrated orally. Enalapril is metabolized only by carboxylesterase (Tocco et al., 1982; Drummer et al., 1990), and the in vitro and in vivo results indicate that enalapril is primarily absorbed by a nonsaturable, passive diffusion process and is not a suitable model compound for studying transporter-related interaction in rats (Morrison et al., 1986). Thus, Enalapril is also considered to be an ideal candidate for testing inhibition of esterase activity with GFJ in the rat model. Evaluation of the esterasemediated changes in the permeability and metabolism in in vitro systems, and in vivo rat exposure to enalapril and lovastatin when coadministered with GFJ are described in this report.

\section{OBJECTIVES}

The objectives of this study were a) to evaluate the effect of GFJ on esterase activity b) to evaluate effect of GFJ on hydrolysis of ester prodrugs enalapril and lovastatin by human intestinal and liver S9, c) to evaluate the effect of GFJ on permeability of enalapril and lovastatin across Caco-2 membrane, d) to evaluate the effect of GFJ on the hydrolysis of enalapril and lovastatin in vitro in rats, e) to evaluate the effect of GFJ on the hydrolysis of enalapril and lovastatin in vivo in rats, and f) to differentiate between CYP3A, esterase, and Pgp effect of GFJ.

\section{MATRIALS AND METHODS}

\subsection{Materials}

GFJ (Minute Maid frozen concentrates) cans were purchased locally (Cambridge, MA); enalapril, para-nitrophenylacetate, p-nitrophenol, phenylmethylsulfonyl 
fluoride (PMSF), bis-p-nitrophenylphosphate (BNPP), glycylsarcosine (Gly-Sar), and 10X Hank's balance buffer were purchased from Sigma (St. Louis, MO); Enalaprilat was purchased from Toroto Research Chemicals Inc. (North York, ON). GF120918 was kindly provided by Dr. Shimoga Prakash. The GFJ used in the study was prepared by 1:3 dilutions with water, unless stated otherwise. Rat liver and intestinal microsomes and S9, and human liver S9 (pool of 50 donors) were purchased from Xenotech (Lenexa, KS); human intestinal S9 (pool of 4 donors) was purchased from Tissue Transformation Technologies (Baltimore, MD); purified porcine esterase was purchased from Sigma (St. Louis, MO); Caco2 cells were obtained from American Type Culture Collection (Manassas, VA); and human PEPT1-expressed transportocytes were purchased from BD Gentest (Woburn, MA).

3.2 The effect of GFJ on purified porcine esterases

Hydrolysis of para-nitrophenylacetate (PNPA)

Purified porcine esterase of $1,2.5$, and 5 munit/ml was incubated at room temperature with $39,78,156,312,625,1250$, and $2500 \mu \mathrm{M}$ PNPA in phosphate buffer $(\mathrm{pH}, 7.4)$ in a final volume of $100 \mu \mathrm{L}$. The brmation of product, paranitrophenol, was monitored spectrophotometrically at $405 \mathrm{~nm}$ at 0,2 , and $4 \mathrm{~min}$.

Effect of GFJ on hydrolysis of para-nitrophenylacetate

Purified porcine esterase ( 5 munit $/ \mathrm{mL}$ ) was incubated at room temperature with PNPA $(0.67 \mathrm{mM})$ in phosphate buffer $(0.1 \mathrm{M} ; \mathrm{pH}, 7.4)$ in the presence or absence of $1: 3$ diluted GFJ ( $\mathrm{pH} 7.4 ; 0,20$ and $40 \%$ final concentration in the incubation mixture) in a final volume of $100 \mu \mathrm{L}$. The formation of product, para-nitrophenol, was monitored spectrophotometrically at $405 \mathrm{~nm}$ at $2 \mathrm{~min}$. 
Effect of GFJ on hydrolysis of enalapril and lovastatin by purified porcine esterases

Purified porcine esterase $(0.1 \mathrm{unit} / \mathrm{mL})$ was incubated at $37^{\circ} \mathrm{C}$ with enalapril and lovastatin $(5 \mu \mathrm{M})$ in $0.1 \mathrm{M}$ potassium phosphate buffer, $\mathrm{pH} 7.4$, in the presence or absence of GFJ ( $\mathrm{pH} 7.4 ; 0,20 \%$ and $40 \%$ ) in an incubation volume of $0.1 \mathrm{~mL}$. Enalapril incubations were quenched after $10 \mathrm{~min}$ and lovastatin incubations after $30 \mathrm{~min}$ by the addition of $10 \mu \mathrm{L}$ of glacial acetic acid. The quenched mixtures were spiked with varied amounts of GFJ to keep the amount of GFJ equal in each sample. The incubation mixtures then were extracted with $100 \mu \mathrm{L}$ of $1 \%$ acetic acid in acetonitrile containing carbutamide as an internal standard. The concentration of enalaprilat and lovastatin acid in the supernatants were determined by LC/MS/MS.

3.3 Effect of GFJ on Hydrolysis of Enalapril and Lovastatin in Human and Rat In Vitro System

Effect of GFJ on hydrolysis of enalapril and lovastatin in human and rat intestinal and liver $\mathbf{S 9}$ fractions.

Human and rat intestinal or liver $\mathrm{S} 9(2.0 \mathrm{mg} / \mathrm{ml})$ were incubated at $37^{\circ} \mathrm{C}$ with enalapril $(5 \mu \mathrm{M})$ in $0.1 \mathrm{M}$ potassium phosphate buffer $(\mathrm{pH} 7.4)$ in the presence or

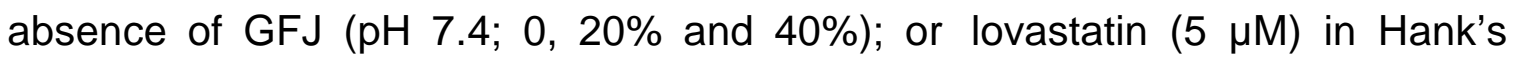
balanced buffer ( $\mathrm{pH} \mathrm{6.0)}$ in the presence or absence of GFJ ( $\mathrm{pH} 6.0 ; 0,20 \%$ and $40 \%$ ). A pH of 6.0 was used to reduce chemical hydrolysis of lovastatin. Liver S9 incubations were carried out for $10 \mathrm{~min}$ and intestinal S9 incubation for $30 \mathrm{~min}$. Reactions were terminated by the addition of $10 \mu \mathrm{L}$ of glacial acetic acid. The incubation mixtures were processed as described in the previous section.

Effect of GFJ on hydrolysis of enalapril and lovastatin in rat intestinal lumen 
Rat lumen was collected by rinsing the small intestine $(30 \mathrm{~cm}$ length from the end of the stomach) of overnight fasted SD rats obtained from Hilltop Laboratory Animals, Inc. (Scottdale, PA) $(\mathrm{n}=3)$ with $1 \times 2 \mathrm{~mL}$ phosphate buffer $(\mathrm{pH}, 7.4)$. The $\mathrm{pH}$ of the pooled lumen was adjusted to 7.0 in a final volume of $7.0 \mathrm{~mL}$. Lumen was used fresh on the day of collection.

A $50 \mu \mathrm{L}$ aliquot of rat lumen was incubated with lovastatin and enalapril $(5 \mu \mathrm{M})$ at $37^{\circ} \mathrm{C}$ in the presence of $50 \mu \mathrm{L}$ of phosphate buffer $(\mathrm{pH}, 7.0$ or 3.5$)$ or $50 \mu \mathrm{L}$ of GFJ $(40 \%$ and $80 \%)$ at $\mathrm{pH}, 7.0$ or 3.5 . After $30 \mathrm{~min}$ incubations, the reactions were terminated by adding an equal volume of acetonitrile containing carbutamide as the internal standard. The samples were centrifuged at $3,000 \mathrm{xg}$ for $10 \mathrm{~min}$. Supernatants were dried under nitrogen and the residues were reconstituted into $0.1 \%$ formic acid in $10 \%$ acetonitrile and analyzed with LC/MS/MS for concentration of lovastatin acid and enalaprilat.

Metabolism of lovastatin in human liver microsomes

Lovastatin $(50 \mu \mathrm{M})$ was incubated with human liver microsomes $(2 \mathrm{mg} / \mathrm{ml})$ in the presence or absence of NADPH $(2.0 \mathrm{mM})$ for $30 \mathrm{~min}$ in an incubation volume of $0.3 \mathrm{~mL}$; reactions were terminated with the addition of an equal volume of acetonitrile. The supernatants were subjected to LC/MS/MS analysis for identifying lovastatin metabolites.

3.4 Effect of GFJ on A-to-B Permeability of lovastatin and enalapril across Caco2 membrane

Caco-2 cells

Caco-2 cells obtained from American Type Culture Collection (Manassas, VA) were cultured in $150-\mathrm{cm}^{2}$ tissue culture T-flasks for subsequent plating onto 24Transwell plate $\left(0.33 \mathrm{~cm}^{2} /\right.$ well, $0.4 \mu \mathrm{M}$ pore size; Costar, Cambridge, MA). 
Briefly $1 \times 10^{5}$ cells were suspended in $0.2 \mathrm{~mL}$ of culture medium (Dulbecco's modified Eagle's medium with $0.1 \mathrm{mM}$ nonessential amino acids, $2 \mathrm{mM} \mathrm{L}$ glutamine, $4.5 \mathrm{~g} / \mathrm{L}$ glucose, and 10\% fetal bovine serum) and added to the upper chamber (Apical) of each filter membrane of a Transwell plate. One milliliter of cell-free culture medium was added to the lower chamber (Basolateral). The Transwell plates were then incubated at $37^{\circ} \mathrm{C}$ in an atmosphere of $5 \% \mathrm{CO}_{2}$ in air and $90 \%$ humidity. The culture media was changed every other day. Confluent cell monolayers were obtained within 5 to 7 days after plating. The transepithelial electrical resistance (TEER), as measured by an epithelial volthommeter (World Precision Instruments, Inc., Sarasota, FL), gradually increased and reached a plateau after day 5 , indicating the formation of tight junctions. Monolayers with TEER values greater than $250 \mathrm{ohm} / \mathrm{cm}^{2}$ were used.

CYP3A and esterase activities of Caco-2 homogenates

Caco-2 cells were harvested by trypsinization. Harvested Caco-2 cells were washed one time with transport buffer and homogenized in transporter buffer. The protein concentration of Caco-2 homogenates was determined using BCA protein assay (Pierce, Rockford, IL). Midazolam or lovastatin $(5 \mu \mathrm{M})$ each was incubated with Caco-2 homogenates $(2 \mathrm{mg} / \mathrm{mL})$ at $37^{\circ} \mathrm{C}$. After $0,10,20$, and 40 min incubation, reactions were terminated by adding an equal volume of acetonitrile containing carbutamide as the internal standard. The concentrations of 1-hydroxy and 4'-hydroxy midazolam (MD) in the supernatants from midazolam incubations; lovastatin and lovastatin acid in the supernatants from lovastatin incubations were determined by LC/MS. Hydroxylovastatin metabolites from lovastatin incubations were identified with LC/MS/MS.

\section{Enalapril and lovastatin A-to-B permeability in Caco-2 cells}

Caco-2 cell cultures were prepared as described above. Single directional transport studies were performed at $37^{\circ} \mathrm{C}$ in air. Prior to each experiment, the confluent cell monolayer on Transwell ${ }^{\mathrm{TM}}$ inserts were washed and equilibrated for 
30 min with transport medium [Hanks' balanced salt solution containing $10 \mathrm{mM}$ $N$-2-hydroxyethylpiperazine- $N$-2-ethanesulfonic acid (HEPES) and $10 \mathrm{mM}$ glucose, $\mathrm{pH}$ 7.4]. The experiment was initiated by adding a solution of lovastatin in the transport medium at $\mathrm{pH} 7.0$ or enalapril in the transport medium at $\mathrm{pH} \mathrm{6.0,}$ containing various proportions of $1: 3$ diluted GFJ $(0,6.2,12.5,25$, and $50 \%$ final concentration in the incubation) to the apical compartment. To evaluate effect of Pgp on lovastatin permeability, GF120918 (2 $\mu \mathrm{M}$ final concentrations in the incubation) shown in Figure 54 was added into lovastatin stock solution to the donor side and the buffer of the receiver side. At 15, 30, 45, and 60 min, aliquots were withdrawn from the basolateral side and replaced immediately with an equal amount of fresh transport media, except at the 60-min time point (the end of the incubation).

After the permeability studies described in the previous section, TEER values were measured again to assure the integrity of the cells. The cells were washed three times with cold transport medium and then were lysed with $1 \%$ acetic acid in water. The cell lysates were extracted with acetonitrile containing carbutamide as internal standard and centrifuged at $3000 \mathrm{xg}$ for $10 \mathrm{~min}$. The supernatants were collected and analyzed using LC/MS/MS

\section{Enalapril uptake in Caco-2 cells}

Prior to the experiment, the confluent cell monolayers on Transwell $^{\mathrm{TM}}$ inserts were washed and equilibrated for 15 min with transport medium, $\mathrm{pH}$ 6.0. Enalapril in transport medium, containing various amount of $\operatorname{GFJ}(0,6.2,12.5$, and $25 \%$ ), was added to the apical compartment. The uptake study was carried out for $5 \mathrm{~min}$ at room temperature and was stopped by aspirating out the solution. The cells were washed three times with cold transport medium and then were lysed with water. The cell lysates were extracted with acetonitrile containing carbutamide as internal standard and centrifuged at $3000 \mathrm{xg}$ for $10 \mathrm{~min}$. The 
supernatants were collected and analyzed using LC/MS/MS for concentrations of the prodrug and the active acid.

\section{Enalapril uptake in human PEPT1-expressed transportocytes}

Enalapril uptake in human PEPT1-expressed transportocytes was performed according to a modified procedure suggested by the manufacture (BD Gentest). Simply, transportocytes (oocytes) were placed in individual wells of 24 well plate (8-10 oocytes in each well) and washed with sodium buffer pH 6.5 three times. Enalpril or Gly-Sar $(20 \mu \mathrm{M})$, a substrate for PEPT1 shown in Figure 54, respectively, in uptake buffer ( $\mathrm{Na}+$ buffer, $\mathrm{pH}$ 6.5) was added into each well to start the uptake under room temperature. After one hour incubation, œcytes were washed with cold sodium uptake buffer three times, and then lysed with acetonitrile containing carbutamide as the internal standard. The cell lysates were centrifuged at $3000 \mathrm{xg}$ for $10 \mathrm{~min}$, and the supernatants were collected and analyzed using LC/MS for the content of Gly-Sar and enalapril, respectively. Water-injected oocytes were used as a negative control; Gly-Sar was used as a positive control.

\section{Binding of Lovastatin to GFJ Pulp}

Lovastatin $(20 \mu \mathrm{M})$ was spiked into transport buffer containing GFJ at various concentrations $(0,6.25,12.5,25.0,50.0$, and 100\%); these samples were centrifuged at $10,000 \times \mathrm{g}$ for $5 \mathrm{~min}$. The content of lovastatin in the supernatants was analyzed by LC/MS/MS.

\subsection{Pharmacokinetic studies in SD rats}

Pharmacokinetics experiments with enalapril and lovastatin were performed in male Sprague-Dawley rats ( $n=3$ or 4 , each group) weighing 280 to $350 \mathrm{~g}$ implanted with either jugular vein cannula or both jugular and portal vein 
cannulas. Animals were fasted overnight and for the duration of the study. Water was provided ad libitum.

For oral application, jugular vein cannulated rats were dosed by gavage (10

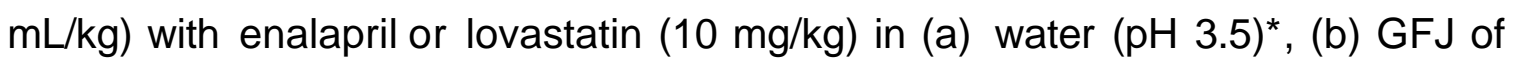
three strengths (1:3 diluted, 1:2 diluted, Concentrate), (c) water, and (d) bis-pnitrophenylphosphate (BNPP) shown in Figure 54 (25 mg/kg, PO). Venous blood samples $(0.3 \mathrm{~mL})$ were collected from jugular vein catheters (JVC) into heparin tubes containing $3 \mu \mathrm{L}$ of $200 \mathrm{mM} \mathrm{PMSF}$ and $5 \mu \mathrm{L}$ of acetic acid $(60 \%$ in water) predose and at $0.25,0.5,1,2,4,6,8$ and $24 \mathrm{~h}$ postdose. Samples were centrifuged for $5 \mathrm{~min}$ at $15000 \mathrm{rpm}$, plasma were collected and frozen at $-80^{\circ} \mathrm{C}$ until analyzed. * Water ( $\mathrm{pH} 3.5$ ) was prepared by spiking glacial acetic acid into water to adjust $\mathrm{pH}$ of water to 3.5 .

For GFJ pretreatment studies, portal vein cannulated (PVC) rats pretreated at 15 and $-2 \mathrm{hr}$ with water (pH 3.5) (Group A), and with GFJ (1:3 diluted, $10 \mathrm{~mL} / \mathrm{kg}$; Groups B and C) were dosed orally with lovastatin (10 mg/kg ) as a solution in water ( $\mathrm{pH}$ 3.5) and in GFJ (1:3 diluted). Portal blood samples were collected from PVC rats predose and at $0.25,0.5,1,2,4,6,8$ and $24 \mathrm{~h}$ postdose. Samples were centrifuged for $5 \mathrm{~min}$ at $15,000 \mathrm{rpm}$, plasma were collected and frozen at $-80^{\circ} \mathrm{C}$ until analyzed.

For intravenous studies, JVC rats were pretreated orally with water $(\mathrm{pH} 3.5)$, or GFJ ( $1: 3$ diluted; $10 \mathrm{~mL} / \mathrm{kg}$ ) at $-0.5 \mathrm{hr}$ prior to a slow IV bolus dose of enalapril at $2 \mathrm{mg} / \mathrm{kg}$ as a saline solution ( $5 \mathrm{~mL} / \mathrm{kg}$ ) or lovastatin at $2 \mathrm{mg} / \mathrm{kg}$ in a 10:40:10:40 mixture of ethanol, polyethylene glycol $400, \mathrm{~N}, \mathrm{~N}$-dimethylacetamide and saline ( 5 $\mathrm{mL} / \mathrm{kg}$ ). Venous blood samples were collected predose and at $0.083,0.25,0.5$, $1,2,4,6,8$ and 24 h postdose.

\section{LC/MS/MS analysis}

The plasma samples were treated with 3 volumes of acetonitrile containing carbutamide $(0.2 \mu \mathrm{M})$ to precipitate protein. Control plasma, with esterase 
inactivated by the addition of $1 \%$ acetic acid and $2 \mathrm{mM}$ phenylmethylsulfonyl fluoride (PMSF) shown in Figure 54, was used to construct plasma standard curves. Half of the volume of supernatant was dried down under nitrogen, and reconstituted with $150 \mu \mathrm{L}$ of $5 \%$ acetonitrile in $0.1 \%$ formic acid, and was analyzed by an LC/MS/MS. The LC/MS/MS system consisted of a binary high performance liquid chromatography pump (1100; Agilent Technologies, Palo Alto, CA), an HTS PAL autosampler (LEAP Technologies, Carrboro, NC), and a triplequadrupole mass spectrometer (API-4000; Applied Biosystems, Foster City, CA). Separation was performed on an YMC-ODS-AQ C18 column $(30 \mathrm{~mm} \times 2.0 \mathrm{~mm}$; Waters, Milford, MA) using mixtures of formic acid (0.1\%) in water and acetonitrile as a mobile phase. The mass spectrometer was operated in the multiple reaction monitoring mode (MRM) using positive ion electrospray ionization. MRM was set at $\mathrm{m} / \mathrm{z} 405.3$ ? 285.3 for lovastatin, $\mathrm{m} / \mathrm{z} 423.3$ ? 303.4 for lovastatin acid, m/z 439.4 ? 283.4 for 6'ß-hydroxylovastatin acid, m/z 421.4 ? 283.4 for 6'B-hydroxylovastatin, $\mathrm{m} / \mathrm{z} 377.1$ ? 233.9 for enalapril, $\mathrm{m} / \mathrm{z} 349.3$ ? 206.4 for enalaprilat, and m/z 272.4 ? 156.2 for carbutamide (internal standard). The concentration range of standard curves for enalapril, enalaprilat, lovastatin

and lovastatin acid, respectively was generally between 1 to $2500 \mathrm{nM}$ with 12 points, and the quantification limit for enalapril, enalaprilat, lovastatin and lovastatin acid was generally $2 \mathrm{nM}$.

Data analysis

The pharmacokinetic parameters were calculated by noncompartmental analysis using WinNonlin Software Version 5.0 (Pharsight, Mountain view, CA). The statistical significance test was performed by using Student T-Test.

\section{RESULTS AND DISCUSSION}

\subsection{Results}


PNPA hydrolysis by purified porcine esterases

PNPA hydrolysis by purified porcine esterase is summarized in Figure 1. At the protein concentration of 5 munit $/ \mathrm{mL}$, linear kinetics was observed at PNPA concentration up to $1.25 \mathrm{mM}$ at $2 \mathrm{~min}$ incubation, thus a concentration of PNPA $0.68 \mathrm{mM}$ was selected for the following inhibition studies.

\section{Effect of GFJ on purified porcine esterases}

Effect of GFJ on the hydrolysis of PNPA, enalapril, and lovastatin by purified porcine esterases, as measured by the formation of the hydrolysis product, is shown in Figure 2. The respective hydrolytic activity with 20 and $40 \%$ GFJ were decreases to $65 \%$ and $54 \%$ for PNPA; $50 \%$ and $31 \%$ for enalapril; and $44 \%$ and $26 \%$ for lovastatin relative to the control values.

Inhibitory effect of GFJ on hydrolysis by human intestinal and liver S9

The percent of lovastatin hydrolyzed was reduced to $54 \%, 46 \%$ and $38 \%$ of the control in human liver S9; $55 \%, 52 \%$ and $47 \%$ of the control in human intestinal S9 using 10\%, 20\% and 40\% GFJ, respectively (Figure 3A). The percent of enalapril hydrolyzed was reduced to $87 \%, 90 \%$ and $78 \%$ of the control in human liver S9 using 10\%, 20\%, and 40\% GFJ, respectively (Figure 3B). Intestinal S9 showed weak hydrolytic activity towards enalapril.

Inhibitory effect of GFJ on hydrolysis of lovastatin and enalapril along GI tract in rats

The percent of lovastatin hydrolyzed was reduced to $78 \%, 65 \%$ and $63 \%$ of the control in rat liver S9; and 55\%, 59\% and $43 \%$ in rat intestinal S9 by $10 \%, 20 \%$ and $40 \%$ GFJ (Figure $4 \mathrm{~A}$ ), respectively. In rat lumen, lovastatin hydrolysis was reduced to $65 \%$ and $48 \%$ of the control by $20 \%$ and $40 \%$ GFJ at pH 7.0 (Figure $4 \mathrm{~B}$ ); and $34 \%$ and $26 \%$ of the control by $20 \%$ and $40 \%$ GFJ at $\mathrm{pH} 3.5$ (pH of 
GFJ). Enalapril hydrolysis was reduced to $80 \%, 68 \%$ and $49 \%$ of the control in rat liver S9; and $83 \%, 70 \%$ and $61 \%$ in rat intestinal S9 by $10 \%, 20 \%$ and $40 \%$ GFJ (Figure 5A), respectively. In rat lumen, the hydrolysis was reduced to $52 \%$ and $45 \%$ by $20 \%$ and $40 \%$ GFJ at $\mathrm{pH} 7.0$ (Figure $5 \mathrm{~B}$ ); and $59 \%$ and $24 \%$ by $20 \%$ and $40 \%$ GFJ at $\mathrm{pH} 3.5$.

CYP3A and esterase activity of Caco-2 homogenates

The CYP3A activity of Caco-2 homogenates was characterized by the formation rate of 1'-hydroxy- and 4-hydroxymidazolam using midazolam as the substrate. As depicted in the Figure 6, the amount of two hydroxyl metabolites formed during the forty minute incubation was negligible that suggests CYP3A activity in this Caco-2 cells is negligible. No hydroxylated metabolites of lovastatin were detected in the lovastatin incubation. Hydrolysis of lovastatin in Caco-2 homogenates (Figure 7 ) showed that $10 \%$ of lovastatin was hydrolyzed to lovastatin acid in the forty minute incubation.

Binding of lovastatin to GFJ pulp

With the increase in the concentration of GFJ, the percentage remaining of lovastatin in the pulp free supernatant was decreased. As summarized in Table 1 , the percentage remaining of lovastatin was $92.6,87.8,74.1,68.2,64.2 \%$ in the presence of GFJ at $6.25,12.5,25,50$, and $100 \%$, respectively.

Effect of GFJ on A-to-B permeability in Caco-2 cells

The permeability of lovastatin was increased by $40 \%$ and $22 \%$ with $6.25 \%$ and $12.5 \%$ GFJ (1:3 diluted), but permeability was decreased by $4 \%$ and $50 \%$ with $25 \%$ and $50 \%$ GFJ, respectively (Figure $8 \mathrm{~A}$ ). In Caco-2 cells at $1 \mathrm{hr}$, the intracellularly trapped lovastatin was decreased to $95 \%, 63 \%, 72 \%$ and $58 \%$; and lovastatin acid was significantly decreased to $71 \%, 40 \%, 29 \%$ and $20 \%$ with 6.25 , 
12.5, 25 and 50\% GFJ, respectively. Overall, the corresponding ratio of lovastatin acid to lovastatin (Figure $8 \mathrm{~B}$ ) was decreased to $81 \%, 64 \%, 42 \%$, and $34 \%$ by GFJ. The permeability of enalapril was significantly increased by $30 \%$, $52 \%, 133 \%$ and $88 \%$ with $6.25 \%, 12.5 \%, 25 \%$ and $50 \%$ GFJ, respectively (Figure 9A). The corresponding intracellularly trapped enalapril in Caco-2 at $1 \mathrm{hr}$ was increased to $139 \%, 156 \%, 162 \%$ and $187 \%$ of the control; and enalaprilat was decreased to $88 \%, 77 \%, 70 \%$ and $68 \%$. Overall, the corresponding ratio of enalaprilat to enalapril (Figure 9B) was decreased to $64 \%, 49 \%, 43 \%$, and $35 \%$ of the control.

Uptake rate of enalapril in Caco-2 cells

Shown in Figure 10A, the uptake rate of enalapril in Caco-2 was 1.71 $\mathrm{pmol} / \mathrm{min} / \mathrm{cm}^{2}$, whereas the values were $1.99,1.91$ and $2.02 \mathrm{pmol} / \mathrm{min} / \mathrm{cm}^{2}$ in the presence of $6.25,12.5$ and $25 \%$ GFJ (1:3 diluted), respectively. The uptake rate of enalapril in Caco-2 in the presence of Gly-Sar (a known PEPT1 substrate; 10 $\mathrm{mM}$ ) did not change significantly, with the value being $1.99 \mathrm{pmol} / \mathrm{min} / \mathrm{cm}^{2}$.

\section{Uptake rate of enalapril in human PEPT1-expressed transportocytes}

Figure 10B shows uptake rate of enalapril and Gly-Sar, a known human PEPT1 substrate in human PEPT1-expressed and water-injected transportocytes. No distinguishable difference of the uptake rate of enalapril between PEPT1expressed and water injected transportocytes was observed. The uptake rate of enalapril is 0.42 and 0.50 in human PEPT1-expressed and water injected transportocytes, respectively. The uptake rate of Gly-Sar is 27.5 and 0.66 in human PEPT1-expressed and water injected transportocytes, respectively.

Identification of lovastatin metabolites in human liver microsomes incubations 
Lovastatin (Figure 51) has two carboxylate groups, a lactone and 2methylbutanoic acid ester. The metabolite from lactone hydrolysis (m/z $405.4+$ 18) was detected in the human microsomal incubation (Figures 11, 14, and 19), but the metabolite resulting from hydrolysis of 2-methylbutanoic acid ester was not detected (Figure 15). Consistent with the published data (Halpin et al. 1993), hydrolysis of lovastatin occurs on the lactone ring. Besides, two hydroxyl metabolite $(\mathrm{m} / \mathrm{z} 405.4+16)$ and two hydroxyacids of the hydroxyl metabolite $(\mathrm{m} / \mathrm{z}$ $405.4+34)$, hydroxylacid, were detected in the human liver microsome incubations (Figures 12, 16, and17). The four metabolites have distinguishable retention time with 5.02 and $6.19 \mathrm{~min}$ for the two hydroxyl metabolites, 4.36 and $5.67 \mathrm{~min}$ for the two hydroxyacid metabolites, respectively. MS/MS data, depicted in Figures 18, 20, 21, 22, and 23, suggested that the peak with $\mathrm{m} / \mathrm{z}$ 421.3 and retention time $(5.02 \mathrm{~min})$ is $6{ }^{\prime}$ ' -hydroxylovastatin, and the peak with $\mathrm{m} / \mathrm{z} 439.4$ and retention time $4.74 \mathrm{~min}$ is its hydroxyacid (6'B-hydroxylovastatin acid). PK plasma samples were analyzed with LC/MS using multiple reaction monitoring mode (MRM). MRM was set at $\mathrm{m} / \mathrm{z} 439.4$ ? 283.4 for 6'Bhydroxylovastatin acid and $421.4 ? 283.4$ for 6'ß-hydroxylovastatin. The retention time of metabolite 1 (439.4 ? 283.4) and metabolite 2 ( 421.4 ? 283.4) found in PK plasma samples was 4.36 and $5.02 \mathrm{~min}$, respectively, consistent with the observations in human liver microsomes incubation.

\section{Effect of BNPP on the PK of enalaprilat and lovastatin acid in rats}

The plasma concentration-time profiles of enalaprilat and lovastatin acid following oral co-administration of enalapril and lovastatin (10 mg/kg), respectively, with water or with BNPP $(25 \mathrm{mg} / \mathrm{kg})$ are shown in Figures $24 \mathrm{~A}$ and $24 \mathrm{~B}$, and the PK parameters are listed in Table 2. Generally, the exposure of rats to both enalaprilat and lovastatin acid was increased by BNPP with the mean $\mathrm{C}_{\max }$ and AUC increased by $56 \%$ and $57 \%$ for enalaprilat, and $235 \%$ and $141 \%$ for lovastatin acid, respectively. 
Effect of GFJ on oral PK of lovastatin acid and enalaprilat in rats

The plasma concentration-time profiles of enalaprilat or lovastatin acid following oral co-administration of enalapril or lovastatin $(10 \mathrm{mg} / \mathrm{kg})$ with water $(\mathrm{pH} 3.5)$ (pH 3.5) or GFJ (1:3 diluted, 1:2 diluted, and the concentrate) are shown in Figures $25 \mathrm{~A}$ and $25 \mathrm{~B}$. The $\mathrm{C}_{\max }$ and $\mathrm{AUC}$ of lovastatin acid were significantly increased in rats following oral administration of lovastatin with GFJ (Table 3). The AUC was increased by $279 \%, 157 \%$ and $170 \%$; and $\mathrm{C}_{\max }$ was increased by $311 \%, 135 \%$ and $157 \%$ upon coadministration with 1:3 diluted, $1: 2$ diluted, and concentrated GFJ, respectively. Similarly the $C_{\max }$ and AUC of enalaprilat were increased in rats following oral coadministration of enalapril with GFJ (1:3 diluted, $1: 2$ diluted, and the concentrate), with the respective AUC increased by $65 \%$, $70 \%$ and $16 \%$ (Table 3); and $\mathrm{C}_{\max }$ increased by $60 \%$ and $50 \%$ (1:3 diluted and $1: 2$ diluted GFJ), and decreased by $43 \%$ by GFJ concentrate, relative to the control.

Effect of GFJ pretreatment on oral PK of lovastatin in portal vein cannulated rats

The portal vein plasma concentration-time profiles of lovastatin acid (Figure 26A), lovastatin acid plus lovastatin (Figure 26B), 6'B-hydroxylovastatin (Figure 27A), and its hydroxy acid (6'B-hydroxylovastatin acid) (Figure 27B) following oral administration of lovastatin $(10 \mathrm{mg} / \mathrm{kg}$ ) to rats pretreated $(-2 \mathrm{hr}$ and $-15 \mathrm{hr}$ ) either with water ( $\mathrm{pH} 3.5$ ) or 1:3 diluted GFJ showed graded effect of GFJ pretreatment. The 6'B-hydroxylovastatin and the corresponding hydroxyacid were identified by LC/MS/MS spectral comparison with the published data (Halpin et al., 1993), and quantitated in plasma using lovastatin standard curves. The portal plasma AUC of lovastatin acid on pretreatment with water $(\mathrm{pH}$ 3.5) (Group A) versus GFJ (Group B) showed 49\% increase in AUC in the GFJ pretreated rats than in water (pH 3.5) (Table 4); and the AUC of the corresponding 6'B-hydroxylovastatin plus 6'B-hydroxylovastatin acid decreased to $12 \%$ in GFJ pretreated rats than pretreated in water ( $\mathrm{pH} 3.5)$ (Table 5). The AUC of lovastatin acid after 
coadministration of lovastatin with GFJ in rats pretreated with GFJ (Group C) showed a $116 \%$ increase compared to pretreatment with water $(\mathrm{pH} \mathrm{3.5)}$. The portal plasma AUC of lovastatin acid plus lovastatin (Group B) showed 22\% increase in AUC in the GFJ pretreated rats than in water (pH 3.5). The AUC of lovastatin acid plus lovastatin after coadministration of lovastatin with GFJ in rats pretreated with GFJ (Group C) showed an $82 \%$ increase compared to pretreatment with water ( $\mathrm{pH} 3.5)$. The AUC of the 6'B-hydroxylovastatin and 6'Bhydroxylovastatin acid was 186 and 83.1 (Group B) and 181 and $77.0 \mathrm{nM}^{*} \mathrm{~h}$ (Group C), respectively. The AUC of the oxidized products was not further reduced upon coadministration with GFJ (Group B vs. C).

Effect of orally dosed GFJ on PK of lovastatin and enalapril after intravenous dosing

The lovastatin acid and lovastatin plasma PK parameters following IV administration of lovastatin $(2 \mathrm{mg} / \mathrm{kg})$ to rats pretreated $(-0.5 \mathrm{~h})$ with water or GFJ (1:3 diluted) are shown in Table 6. Similar PK data for enalapril are shown in Table 7. The PK parameters did not change between the two pretreatment groups for both drugs.

\subsection{Discussion}

It is well known that GFJ inhibits the intestinal CYP3A4 (Schmiedlin-Ren et al. 1997; He et al., 1998), and also the Pgp, MRP2 and OATP (Honda et.al, 2004, Dresser et al., 2002). The literature is replete with examples of drug interactions with CYP3A (Saito et al., 2005). For the first time, we demonstrate in this study, that GFJ also inhibits the esterase, specifically carboxylesterase (CE), activity in vitro in rats and humans and in vivo in rats. Carboxylesterases are ubiquitous enzymes responsible for the metabolism of xeno- and endo-biotics (Williams et al., 1985). Though other fruit juices are shown to inhibit esterase activity, the literature lacks reports on esterase inhibition by GFJ. This report provides the 
proof of concept with the demonstration of inhibitory effect of GFJ on PNPA and the prodrugs lovastatin and enalapril using purified porcine esterase. Lovastatin is known to be hydrolyzed by human carboxylesterase (hCE1/2) to its active acid form and to be oxidized by CYP3A (Halpin et al., 1993). A recent study (Chen et al., 2005) also suggested that lovastatin is a weak substrate of Pgp. Thus, the modes of interaction of GFJ with lovastatin in vivo could be CYP3A inhibition, esterase inhibition, and or Pgp inhibition. Enalapril, on the other hand, is a substrate only for carboxylesterase (hCE1) (Tocco et al., 1982; Drummer et al., 1990). The inhibition of hCE1 could lead to increased stability of esters in the gut enterocytes and lumen, resulting in greater absorption of esters, and hence higher exposure to the active acids, via rapid hydrolysis in plasma, for lovastatin and enalapril.

\section{Esterase inhibition in vitro}

The hydrolysis of these esters (measured by product formation) by purified porcine esterase was inhibited by GFJ in a concentration dependent manner (Figure 2). GFJ significantly inhibited the hydrolysis of lovastatin and enalapril also in rat intestinal lumen, and rat and human intestinal and/or liver S9, as depicted in Figures 3 - 5. The Caco-2 system was utilized to check if the increased stability of prodrugs in the gut by GFJ could lead $b$ enhancement of the permeability. Caco-2 cells used were determined to contain only a minor CYP3A activity, as indicated in Figure 6. Thus, effects due to Pgp could be sorted out for lovastatin using this model. GF120918, a Pgp and BCRP inhibitor (Xia et al., 2005), failed to significantly alter lovastatin permeability suggesting that the contribution of Pgp to lovastatin permeability was negligible. GFJ led to enhancement of $A \rightarrow B$ permeability of lovastatin and enalapril (Figures $8 \mathrm{~A}$ and 9A). Thus, it is mainly the esterase inhibition attribute of GFJ that led to the

higher permeability of lovastatin. As expected, in these experiments the intracellularly trapped lovastatin acid and enalaprilat were reduced by GFJ (Figures 8B and 9B). The amount of lovastatin acid and enalaprilat on the donor 
side at the end of the incubation was very low and was not affected by GFJ. The published in vitro and in vivo results (Morrison et al., 1986) indicate that enalapril is primarily absorbed by a non-saturable, passive diffusion process and it is not a suitable model compound for studying dipeptide transporter interaction in rats. Our data (Results section) confirmed that GFJ indeed did not affect the uptake of enalapril (Figures 10A and 10B).

\section{Esterase inhibition in vivo}

In vivo, the exposure to lovastatin acid was increased by $279 \%, 157 \%$ and $170 \%$ in rats following oral administration of lovastatin with 1:3 diluted, 1:2 diluted and concentrated GFJ, respectively, compared to dosing with water ( $\mathrm{pH} 3.5$ ) (Table 2). At higher concentrations of GFJ, however, a drop in exposure was observed. This is believed to be due to trapping/binding of the drug particles by GFJ pulp, which makes less of free drug available for absorption when higher strengths of GFJ are used. This hypothesis was tested by spiking a constant amount of drug in GFJ of various strengths followed by mixing and centrifugation. The concentration of the drug in the centrifugate (pulp free) was much lower at higher GFJ strengths, as suggested in Table 1, supporting our hypothesis. BNPP, a known esterase inhibitor (Walker et al., 1983), produced an effect similar to GFJ (Tables 2). The exposure to lovastatin acid was increased by $141 \%$ in rats following oral coadministration of lovastatin with BNPP compared to coadministration with water. The positive BNPP effect suggested that the esterase plays a significant role in modulating the oral exposure to lovastatin. Thus, the large increase in AUC observed with GFJ was a combination of its effects due to CYP3A and esterase inhibition. In order to differentiate the modes of GFJ effects via CYP3A inhibition and esterase inhibition, portal veincannulated rats were pretreated with GFJ at -15 and $-2 \mathrm{hr}$ of lovastatin administration to achieve a maximal inactivation of CYP3A activity. 6'Bhydroxylovastatin, the major CYP3A-mediated metabolite (Vyas et al., 1990) and its hydroxy acid (6'B-hydroxylovastatin acid) in portal plasma were determined to 
qualitatively assess the magnitude of intestinal CYP3A activity involved. The portal exposure of 6'B-hydroxylovastatin and 6'B-hydroxylovastatin acid was significantly decreased to $\sim 11 \%$ (Figures $27 \mathrm{~A}$ and 27B; Table 5), as expected, upon pretreatment with GFJ compared to the pretreatment with water. As a result of inhibition of CYP3A by GFJ pretreatment, the portal plasma exposure to lovastatin acid was increased by $49 \%$ (Table. 4, Groups A and B). When lovastatin was coadministered with GFJ (Group C) to portal vein cannulated rats pretreated with GFJ at -15 and $-2 \mathrm{hr}$, the portal plasma exposure to CYP3A mediated products 6'B-hydroxylovastatin and 6'B-hydroxylovastatin acid was generally unchanged, compared to Group $B$ rats, as shown in Table 5, indicating that the CYP3A activity remained inhibited by the pretreatment regimen. However, the portal plasma AUC of lovastatin acid in rats coadministered with GFJ (Group C) was further increased by $45 \%$ (Table 4 ). The additional $45 \%$ exposure increase in Group $\mathrm{C}$ is postulated to be the results of inhibition of gut lumen and enterocyte esterase by the co-administered GFJ. The quantitation of lovastatin plus lovastatin acid showed a similar trend. Thus, both CYP3A inhibition and esterase inhibition attributes of GFJ led to the exposure increases for lovastatin and the active drug lovastatin acid in rats.

The exposure to enalaprilat was similarly increased by 65 and $70 \%$ in rats following oral coadministration of enalapril with $1: 3$ and 1:2 diluted GFJ, compared to coadministration with water alone (Figure 25A, Table 3). Enalapril is only metabolized by carboxylesterase (hCE1), and is not a CYP3A substrate, thus the esterase inhibition by GFJ led to oral exposure increase of enalaprilat in rats. This is consistent with the increased exposure of enalapril when coadministered with the esterase inhibitor BNPP (Table 2), as well as with the in vitro data. When GFJ was administered orally $0.5 \mathrm{~h}$ prior to the IV administration of lovastatin and enalapril to rats the IV pharmacokinetic parameters remained unaltered, indicating that single oral administration of GFJ did not alter the esterase activity at the hepatic level. 


\section{CONCLUSIONS}

The current study, for the first time, demonstrated that GFJ decreased lovastatin and enalapril hydrolysis in the gut, and thereby markedly increased metabolic stability and permeability of esters, leading to the enhancement of exposure to lovastatin acid and enalaprilat in rats. The esterase inhibition by GFJ similarly could also lead to oral exposure increase of lovastatin acid and enalaprilat in humans. Moreover, it is proposed that other ester prodrugs are also likely to show drug interactions mediated via esterase inhibition attribute of GFJ in the clinic. 
PART II

ESTERASE INHIBITION BY GRAPEFRUIT JUICE FLAVONIDS LEADS TO A NEWLY IDENTIFIED DRUG INTERACTION 


\section{INTRODUCTION}

Since the first report of the GFJ effect on the oral bioavailability of felodipine (Bailey et al., 1989, 1991), many studies for identifying the active components responsible for the GFJ effects have been reported (Bailey et al., 1993, Edwards et al., 1996; Fukuda et al. 1997; Schmiedlin-Ren et al., 1997, He et al., 1998; Guo et al., 2000; Ho et al., 2001). GFJ composition varies from variety to variety and from lot to lot and also depends on the preparation method (De Castro et al., 2006). In all cases, the majority of the constituents are flavonoids (Ho et al., 2000). Naringin, a predominant constituent in GFJ, is present in concentrations up to $2000 \mu \mathrm{M}$ (Ross et al., 2000). Even relatively low abundance flavonoids, such as quercetin, occur in the $20 \mu \mathrm{M}$ range. Bergamottin and 6',7'dihydroxybergamottin, the most abundant furanocoumarin derivatives, are well studied GFJ constituents that are present in concentrations up to $40 \mu \mathrm{M}$ (Edwards et al., 1996). Although many bioflavonoids inhibit CYP3A in vitro (Ho et al., 2001), in vivo, naringin alone at concentrations found in GFJ was not capable of producing a clinical drug interaction such as seen with grapefruit juice (Bailey et al., 1998). Several furanocoumarins in grapefruit juice are effective in vitro CYP3A inhibitors and are currently suggested to be clinically active constituents (Schmiedlin-Ren et al., 1997, He et al., 1998).

In Part I, we demonstrated that GFJ inhibits esterase activity and mediates pharmacokinetic interaction with ester prodrugs lovastatin and enalapril, It is important to identify the active components responsible for this new esterasemediated GFJ effects in vivo. Several classical esterase inhibitors are known including diethyl p-nitrophenyl phosphate (Walker et al., 1983) and bis-pnitrophenylphosphate (BNPP) (Gratzl et al., 1975). However, the extreme toxicity of these compounds precludes their clinical use. Recently, a series of synthesized benzene sulfonamides and the aromatic dione family were identified as selective inhibitors of CEs (Wadkins et al., 2004 and 2005). Likewise, flavoring ester mixtures in strawberry juice were also reported to interact with the 
prodrug of tenofovir leading to enhanced permeability across Caco-2 cells (Van Gelder et al., 2002). In this study, the esterase inhibition potential of ten grapefruit juice components towards PNPA hydrolysis as well as the effect of two selected components on the esterase mediated changes in the permeability in in vitro systems, and in in vivo rat exposure to active acids of enalapril and lovastatin upon coadministration are described.

\section{OBJECTIVES}

The objectives of this study were a) to identify grapefruit juice components responsible for the new esterase substrate-GFJ drug interactions, b) to evaluate esterase inhibition potential of the identified GFJ components (s) on hydrolysis of ester prodrugs enalapril and lovastatin by human liver microsomes, c) to evaluate effects of the identified constituent esterase inhibitor(s) on permeability of enalapril and lovastatin across Caco-2, d) to evaluate effects of the identified esterase inhibitor(s) on hydrolysis of enalapril and lovastatin in vitro in SD rats, e) to evaluate effects of the identified esterase inhibitor(s) on oral exposure of enalapril and lovastatin in SD rats in vivo, and f) to differentiate between CYP3A, esterases, and Pgp effects of the identified active components.

\section{MATERIALS AND METHODS}

\subsection{Materials}

Enalapril, lovastatin, para-nitrophenyl acetate (PNPA), p-nitrophenol, phenylmethylsulfonyl fluoride (PMSF), bis-p-nitrophenylphosphate (BNPP), bergapten, kaempferol, quercetin, morin, galangin, naringenin, naringin, and hesperidin were purchased from Sigma (St. Louis, MO); bergamottin and 6',7'dihydroxybergamottin $\left(6^{\prime}, 7^{\prime}-\mathrm{DHB}\right)$ were purchased from BD Gentest (Woburn, MA); enalaprilat and lovastatin hydroxy acid were purchased from Toronto Research Chemicals Inc. (North York, ON). Human liver microsomes (pool of 50 
donors) were purchased from Xenotech (Lenexa, KS); and purified porcine esterase was purchased from Sigma (St. Louis, MO).

11.2 Effect of GFJ chemical constituents on purified porcine esterases

Purified porcine liver esterase ( 5 munit $/ \mathrm{mL}$ ) in $0.1 \mathrm{M}$ potassium phosphate buffer $\mathrm{pH} 7.4$ was incubated at $37^{\circ} \mathrm{C}$ with PNPA $(667 \mu \mathrm{M})$ and one of the ten GFJ components at ten different concentrations; bergamottin $(0 \sim 100 \mu \mathrm{M})$, bergapten (0 $100 \mu \mathrm{M}), 6^{\prime}, 7^{\prime}$-dihydroxybergamottin $(0 \sim 100 \mu \mathrm{M})$, kaempferol $(0 \sim 50 \mu \mathrm{M})$, quercetin $(0 \sim 50 \mu \mathrm{M})$, morin $(0 \sim 50 \mu \mathrm{M})$, galangin $(0 \sim 50 \mu \mathrm{M})$, naringenin $(0 \sim$ $200 \mu \mathrm{M})$, hesperidin $(0 \sim 200 \mu \mathrm{M})$, and naringin $(0 \sim 1000 \mu \mathrm{M})$. Their structures are shown in Figure 53. The highest concentration of each component used for esterase inhibition evaluation varied based on their solubility in incubation buffer and their concentration found in GFJ. The formation of the product, paranitrophenol, was monitored spectrophotometrically at $405 \mathrm{~nm}$ at $2 \mathrm{~min}$. BNPP (0 20 $\mu \mathrm{M})$ was used as a positive control.

11.3 Effect of GFJ chemical constituents on esterases in human liver microsmes

Hydrolysis of PNPA by human liver microsomes

Human liver microsomes at 0.25 , and 0.5 , and $1.0 \mathrm{mg} / \mathrm{mL}$ were incubated at room temperature with $0.67 \mathrm{mM}$ para-nitrophenylacetate in phosphate buffer $(\mathrm{pH}$, 7.4) in a final volume of $100 \mu \mathrm{L}$. The formation of product, para-nitrophenol, was monitored spectrophotometrically at $405 \mathrm{~nm}$ for $0,1,2,3$, and $4 \mathrm{~min}$. The results are summarized in Figure 28.

Effect on esterase activity in human liver microsomes

Human liver microsomes $(0.1 \mathrm{mg} / \mathrm{mL})$ were incubated at room temperature with $0.67 \mathrm{mM}$ PNPA in phosphate buffer $(\mathrm{pH}, 7.4)$ and one of the ten GFJ components at ten different concentrations as described in the section above. 
The formation of product para-nitrophenol was monitored spectrophotometrically at $405 \mathrm{~nm}$ at $2 \mathrm{~min}$.

11.4 Effect of GFJ chemical constituents on A-to-B permeability of lovastatin and enalapril across Caco-2 membrane

Caco-2 cell cultures were prepared as described in Part I, section 2.3. Single directional transport studies were performed at $37^{\circ} \mathrm{C}$ in air. Prior to each experiment, the confluent cell monolayer on Transwell ${ }^{\mathrm{TM}}$ inserts were washed and equilibrated for 30 min with transport medium [Hanks' balanced salt solution containing $10 \mathrm{mM} \mathrm{N}$-2-hydroxyethylpiperazine- $N$-2-ethanesulfonic acid (HEPES) and $10 \mathrm{mM}$ glucose, $\mathrm{pH}$ 7.4]. The experiment was initiated by adding a solution of lovastatin in the transport medium at $\mathrm{pH} 7.0$ or enalapril in the transport medium at $\mathrm{pH}$ 6.0, containing various amount of grapefruit components to the apical compartment. Both lovastatin stock solution in the donor side and buffer in the receiver side contained GF120918 (2 $\mu \mathrm{M}$ final concentrations in the incubation) to assess Pgp effect on lovastatin permeability. At 15, 30, 45, and 60 min, aliquots were withdrawn from the basolateral side and replaced immediately with an equal amount of fresh transport media, except at the 60-min time point (the end of the incubation). After the permeability studies described in the previous section, TEER values were measured again to assure the integrity of the cells. The cells were washed three times with cold transport medium and then were lysed with $1 \%$ acetic acid in water. The cell lysates were extracted with acetonitrile containing carbutamide as internal standard and centrifuged at $3000 \times \mathrm{xg}$ for $10 \mathrm{~min}$. The supernatants were collected and analyzed using LC/MS/MS.

\subsection{Pharmacokinetic studies}


Pharmacokinetics experiments with enalapril and lovastatin were performed in male Sprague-Dawley rats ( $n=3$ or 4 , each group) (Hilltop Laboratory, PA) weighting 280 to $350 \mathrm{~g}$ implanted with either jugular vein cannula or both jugular and portal vein cannulas. Animals were fasted overnight and for the duration of the study. Water was provided ad libitum.

For systemic exposure studies, jugular vein cannulated rats were orally dosed by gavage with enalapril and lovastatin (10 mg/kg, $10 \mathrm{ml} / / \mathrm{kg}$ ) in (a) water, (b) kaemferol (2 and $10 \mathrm{mg} / \mathrm{kg}$ ); and (C) naringenin (2 and $10 \mathrm{mg} / \mathrm{kg}$ ). Venous blood samples $(0.3 \mathrm{~mL})$ were collected from jugular vein catheters (JVC) into heparin tubes containing $3 \mu \mathrm{L}$ of $200 \mathrm{mM}$ PMSF and $5 \mu \mathrm{L}$ of acetic acid $(6: 4$ diluted with water) predose and at $0.25,0.5,1,2,4,6,8$ and $24 \mathrm{~h}$ postdose. Samples were centrifuged and plasma samples were collected and frozen at $80^{\circ} \mathrm{C}$ until analyzed.

For portal exposure studies, portal vein cannulated (PVC) rats orally dosed by gavage with lovastatin (10 mg/kg, $10 \mathrm{ml} / \mathrm{kg}$ ) formulated in (a) water and (b) kaempferol $(10 \mathrm{mg} / \mathrm{kg})$. Portal blood samples $(0.3 \mathrm{~mL})$ were collected into heparin tubes containing $3 \mu \mathrm{L}$ of $200 \mathrm{mM}$ PMSF and $5 \mu \mathrm{L}$ of acetic acid (6:4 diluted with water) predose and at $0.25,0.5,1,2,4,6,8$ and $24 \mathrm{~h}$ postdose. Samples were centrifuged and plasma samples were collected and frozen at $80^{\circ} \mathrm{C}$ until analyzed.

\section{LC/MS/MS Analysis}

In vivo plasma samples were protein precipitated and analyzed with an LC/MS/MS method. Blank plasma, in which esterase was inactivated with $1 \%$ acetic acid and $2 \mathrm{mM}$ PMSF was used to construct plasma standard curves. In general, to one volume of plasma, were added 3 volumes of acetonitrile containing carbutamide $(0.2 \mu \mathrm{M})$ as the internal standard. Samples were vortexed and then centrifuged for $15 \mathrm{~min}$ at $3000 \mathrm{~g}$. Half of the supernatant was dried down under nitrogen, and reconstituted with $150 \mu \mathrm{L}$ of $5 \%$ acetonitrle in $0.1 \%$ formic acid. The LC/MS/MS system consisted of a binary high 
performance liquid chromatography pump (1100; Agilent Technologies, Palo Alto, CA), an HTS PAL autosampler (LEAP Technologies, Carrboro, NC), and a triplequadrupole mass spectrometer (API-4000; Applied Biosystems, Foster City, CA). Separation was performed on an YMC-ODS-AQ C18 column $(30 \mathrm{~mm} \times 2.0 \mathrm{~mm}$; Waters, Milford, MA) using mixtures of formic acid (0.1\%) in water and acetonitrile as a mobile phase. The mass spectrometer was operated in the multiple reaction monitoring mode (MRM) using positive ion electrospray ionization. MRM was set at m/z 405.3 ? 285.3 for Lovastatin, 423.3 ? 303.4 for lovastatin acid, 421.4 ? 283.4 for 6'B-hydroxylovastatin, 377.1 ? 233.9 for enalapril, 349.3 ? 206.4 for enalaprilat and $\mathrm{m} / \mathrm{z} 272.4$ ? 156.2 for carbutamide (internal standard). The concentration range of standard curves for enalapril, enalaprilat, lovastatin and lovastatin acid, respectively was generally between 1 to $2500 \mathrm{nM}$ with 12 points, and the quantification limit for enalapril, enalaprilat, lovastatin and lovastatin acid was generally $2 \mathrm{nM}$.

\section{Data Analysis}

Percent inhibition of PNPA hydrolysis for each inhibitor was calculated as the ratio of $O D$ at each concentration of inhibitor with respect to that in the absence of the inhibitor, and the percentages were plotted against the concentrations of each tested inhibitor using Prism software (GraphPad, San Diego, CA). The sigmoidal dose-response (variable slope) model was used to determine the concentration which gave $50 \%$ inhibition $\left(\mathrm{IC}_{50}\right)$. The $\mathrm{IC}_{50}$ was calculated using the equation

$\mathrm{Y}=$ Minimum activity $+($ Maximum activity - Minimum activity $) /\left(1+10 \mathrm{e}^{(\log E C 50-}\right.$ X)*HillSlope)

where $\mathrm{X}$ is the logarithm of concentration, and $\mathrm{Y}$ is the percent activity. 
PK parameters were calculated by noncompartmental analysis WinNonlin software Version 5.1 (Pharsight, Mountain view, CA). Statistical analysis was using student $t$ test.

\section{RESULTS AND DISCUSSIONS}

\subsection{Results}

Kinetics of PNPA hydrolysis by human liver microsomes

At PNPA concentrations up to $0.68 \mathrm{mM}$, the formation of para-nitrophenol was linear up to 2 minutes incubation at protein concentrations of $0.025,0.05$, and 0.1 $\mathrm{mg} / \mathrm{mL}$, respectively.

Effect of GFJ chemical components on purified porcine esterases

The inhibition curves of ten GFJ components and BNPP on purified porcine esterases are shown in Figures 29-32. Their inhibitory potential towards esterase activity varied widely (Table 8). Bergamottin, 6',7'-DHB and bergapten (each at $100 \mu \mathrm{M}$ ), hesperidin at $200 \mu \mathrm{M}$, and naringin at $1000 \mu \mathrm{M}$ did not show appreciable inhibition of the hydrolysis of PNPA by porcine liver esterase. However, morin, galangin, kaempferol, quercetin, and naringenin all showed an inhibitory effect. Estimates of $\mathrm{IC}_{50}$ were $1.8 \mu \mathrm{M}$ for morin, $2.8 \mu \mathrm{M}$ for galangin, $5.1 \mu \mathrm{M}$ for kaempferol, $5.9 \mu \mathrm{M}$ for quercetin, and $110 \mu \mathrm{M}$ for naringenin. The value of $\mathrm{IC}_{50}$ for BNPP was $1.1 \mu \mathrm{M}$.

Effect of GFJ chemical components on PNPA hydrolysis by human liver microsomes

The inhibition curves of ten GFJ components and BNPP on PNPA hydrolysis in human liver microsomes are shown in Figures 33-36. Likewise, in human liver 
microsomes bergamottin, 6', 7'-DHB, bergapten, naringin and hesperidin did not appreciably inhibit the hydrolysis of PNPA (Table 9). Estimates of $\mathrm{IC}_{50}$ for human liver microsomes were $80 \mu \mathrm{M}$ for morin, $81 \mu \mathrm{M}$ for galangin, $62 \mu \mathrm{M}$ for kaempferol, $43 \mu \mathrm{M}$ for quercetin, $30 \mu \mathrm{M}$ for naringenin. The value of $\mathrm{IC}_{50}$ for BNPP was $11.9 \mu \mathrm{M}$.

Effect of kaempferol and naringenin on A-to-B permeability in Caco-2 cells

The permeability of lovastatin was not altered by GF120918, a Pgp and BCRP inhibitor, and was increased by $65 \%, 64 \%$, and $66 \%$ by kaempferol $(250 \mu \mathrm{M})$, naringenin $(250 \mu \mathrm{M})$, and the mixture of kaempferol $(250 \mu \mathrm{M})$ and GF120918 (2 $\mu \mathrm{M}$; Figure 37A). In Caco-2 cells at $1 \mathrm{hr}$ the intracellularly trapped lovastatin was not significantly altered, with the respective values of $117 \%, 96 \%, 105 \%$, and $116 \%$ of the control, whereas the trapped amount of lovastatin acid was unaffected by GF120918 and decreased to $55 \%, 29 \%$, and $52 \%$ by kaempferol, naringenin, and mixture of kaempferol and GF120918. The overall ratios of lovastatin acid to lovastatin in Caco-2 cells were decreased and are shown in Figure 37B. The permeability of enalapril was increased by $106 \%, 79 \%, 188 \%$, and $219 \%$ with kaempferol (50 and $250 \mu \mathrm{M}$ ) and naringenin (50 and $250 \mu \mathrm{M}$ ), respectively (Figure 38A). The corresponding intracellularly trapped enalapril in Caco-2 cells at $1 \mathrm{hr}$ was increased by $67 \%, 69 \%, 26 \%$, and decreased 11\%; and enalaprilat was decreased to $55,37,58$, and $40 \%$. The overall ratios of enalaprilat to enalapril were decreased and are shown in Figure 38B. The amount of lovastatin acid and enalaprilat on the donor side at the end of the incubation was very low and was not affected by kaempferol and naringenin.

\section{Effect of kaempferol and naringenin on oral PK of enalaprilat in rats}

The plasma concentration-time profiles of enalaprilat in rats following oral coadministration of enalapril (10 mg/kg) with water, kaempferol (2 and $10 \mathrm{mg} / \mathrm{kg}$ ), 
and naringenin ( 2 and $10 \mathrm{mg} / \mathrm{kg}$ ) are shown in Figures 39A and 39B. The PK data are shown in Table 10.

Effect of kaempferol and naringenin on oral PK of lovastatin acid in rats

The plasma concentration-time profiles of lovastatin acid in rats following oral co-administration of lovastatin (10 mg/kg) with water, kaempferol (2 and 10 $\mathrm{mg} / \mathrm{kg}$ ), and naringenin (2 and $10 \mathrm{mg} / \mathrm{kg}$ ) are shown in Figures $40 \mathrm{~A}$ and $40 \mathrm{~B}$. The PK data are shown in Table 11.

Effect of kaempferol on the portal plasma PK of lovastatin

The portal vein plasma concentration-time profiles of lovastatin, lovastatin acid, and the major oxidative product, 6'ß-hydroxylovastatin, following oral coadministration of lovastatin $(10 \mathrm{mg} / \mathrm{kg})$ to rats with water or kaempferol $(10 \mathrm{mg} / \mathrm{kg})$ are shown in Figures 41A, B, and C. The 6'B-hydroxylovastatin was identified by LC/MS/MS spectral comparison with published data (Halpin et al., 1993), and quantitated in plasma using lovastatin standard curves. The portal plasma PK parameters are shown in Table 12. The AUC ratio of 6'B-hydroxylovastatin and lovastatin was 0.064 and 0.078 to rats upon coadministration of lovastatin with water and kaempferol, respectively.

\subsection{Discussions}

Esterase inhibition in vitro

The ten grapefruit juice chemical components investigated in this study belong to two major and widely known classes, flavo noids (aglycones and glycosides) and furanocoumarins. In the earlier years, grapefruit flavonoids were extensively studied for CYP3A inhibition potential to better understand the GFJ effect on oral availability of CYP3A substrates. Though these flavonoids in vitro inhibited 
CYP3A4 but did not reproduce the grapefruit juice effect when administered orally (Rashid et al. 1993, Bailey et al. 1993). Currently, it is believed that grapefruit furanocoumarins, bergamottin and 6',7'-dihydroxybergamottin in GFJ are responsible for the GFJ interaction by competitive and mechanism-based inhibition of CYP3A in the small intestine. These two potent CYP3A inhibitors, in the current study, were found to be devoid of esterase inhibitory activity at 100 $\mu \mathrm{M}$, a concentration higher than that found with GFJ. Some of the flavonoids on the other hand were found to have esterase inhibitory activity. The effect of flavonoids in aglycone form on carboxylesterases is distinguishably different from flavonoids in glycoside form. Naringin and hesperidin, the two glycosidic flavonoids, did not inhibit PNPA hydrolysis at concentrations found in GFJ. However, the five flavonoids morin, galangin, kaempferol, quercetin, and naringenin, in aglycone forms, markedly inhibited PNPA hydrolysis with $\mathrm{IC}_{50}$ values in the low $\mu \mathrm{M}$ range, 1.8 to $110 \mu \mathrm{M}$. The combined effects of flavonoids could contribute significantly to the GFJ effects on PK of ester prodrugs. The effect of kaempferol and naringenin on enalapril and lovastatin permeability in Caco-2 model and PK in rats was examined. Kaempferol was reported to inhibit CYP3A and Pgp in vitro (Jignesh et al., 2004); Caco-2 cells used in our study were determined to contain only a minor CYP3A activity (Figure 6). Thus, effects due to Pgp could be sorted out readily for lovastatin, a prodrug metabolized by CYP3A as well as carboxylesterase, using this model. Kaempferol and naringenin led to enhancement of $A \rightarrow B$ permeability of lovastatin and enalapril (Figures 37A and 38A). GF120918, a Pgp and BCRP inhibitor (Xia et al., 2005), failed to significantly alter lovastatin permeability suggesting that the contribution of Pgp to lovastatin's permeability was negligible. Thus, it is mainly the esterase inhibition attribute of kaempferol and naringenin that led to the higher permeability of lovastatin and enalapril. As expected, in these experiments the intracellularly trapped lovastatin acid and enalaprilat were reduced by kaempferol and naringenin, as indicated by decreases in the ratios of lovastatin acid $b$ lovastatin to $59 \%, 27 \%$ and $45 \%$ by kaempferol, naringenin and a mixture of kaempferol and GF120918 (Figure 37B), and enalaprilat to enalapril to 27\%, 19\%, 
$44 \%$ and $38 \%$ by kaempferol ( 50 and $250 \mu \mathrm{M}$ ) and naringenin (50 and $250 \mu \mathrm{M}$ ), respectively (Figures 38B).

\section{Esterase inhibition in vivo}

The AUC of lovastatin acid was increased by $171 \%, 246 \%, 159 \%$, and $288 \%$ in rats following oral administration of lovastatin with kaempferol (2 and $10 \mathrm{mg} / \mathrm{kg}$ ) and naringenin ( 2 and $10 \mathrm{mg} / \mathrm{kg}$ ), respectively, compared to dosing with water (Table 11). BNPP, a known esterase inhibitor (Walker et al., 1983), produced an effect similar to kaempferol and naringenin, as described in Part I The positive BNPP effect suggested that the esterase plays a significant role in modulating the oral exposure to lovastatin. Thus, the large increase in AUC observed with kaempferol and naringenin was a combination of their effects due to CYP3A and esterase inhibition. In order to differentiate the modes of kaempferol and naringenin effects via CYP3A inhibition, esterase inhibition, and/or combination

of both, portal vein-cannulated rats were dosed with water or with kaempferol (10 $\mathrm{mg} / \mathrm{kg}$ ). The portal plasma exposure of lovastatin acid, lovastatin, and 6'Bhydroxylovastatin with kaempferol showed $113 \%, 154 \%$, and $208 \%$ higher AUC than dosing with water. Lovastatin is known to be metabolized by CYP3A to 6'Bhydroxylovastatin as one of the major oxidized products (Halpin et al., 1993). The possible effect of kaempferol on lovastatin exposure by inhibiting intestinal CYP3A was examined by comparing AUC ratio of oxidized metabolite 6'Bhydroxylovastatin to lovastatin. The 6'B-hydroxylovastatin was identified by LC/MS/MS spectral comparison with the published data (Halpin et al., 1993), and quantitated in plasma using lovastatin standard curves. The AUC ratio of 6'Bhydroxylovastatin to lovastatin was 0.064 and 0.078 for coadministration with water and kaempferol, respectively (Table 12) indicating that kaempferol did not markedly inhibit intestinal CYP3A activity in rats. This finding is consistent with the literature that kaempferol and naringenin are weak CYP3A inhibitors with $\mathrm{IC}_{50}$ values greater than $100 \mu \mathrm{M}$ (Ho et al., 2001). 
The exposure to enalaprilat was also increased by $60 \%, 109 \%, 18 \%$, and $38 \%$ in rats following oral administration of enalapril with kaempferol (2 and $10 \mathrm{mg} / \mathrm{kg}$ ) and naringenin (2 and $10 \mathrm{mg} / \mathrm{kg}$ ), respectively, compared to dosing with water (Figures 39A and 39B, Table 10). Enalapril is only metabolized by carboxylesterase (hCE1), and is not a CYP3A substrate, thus the esterase inhibition by kaempferol and naringenin largely led to oral exposure increase of enalaprilat in rats. This is consistent with the increased exposure of enalapril when coadministered with the esterase inhibitor BNPP, as seen in Part I, as well as with the in vitro data.

\section{CONCUSIONS}

This study identified GFJ flavonoids as esterase inhibitors, and these flavonoids maybe the active components responsible for esterase inhibitory activity of GFJ. Two of the flavonoids kaempferol and naringenin showed significant exposure

changes to the active acids in rats when coadministered with lovastatin and enalapril. These flavonoids have the potential of being used clinically as boost agents to selectively inhibit esterase for enhancing the absorption of ester prodrugs. 


\section{PART III}

THE INTERACTION MECHANISM OF GRAPEFRUIT JUICE CHEMICAL CONSTITUENTS WITH ESTERASES 


\section{INTRODUCTION}

Several classical esterase inhibitors are known in the literature, including diethyl p-nitrophenyl phosphate (paraxon) (Walker et al., 1983), and bis-pnitrophenylphosphate (Gratzl et al., 1975) (BNPP). Recently, a series of benzene sulfonamides were identified as selective inhibitors of human carboxylesterase (hCEs), and the aromatic dione family (Wadkins et al., 2004 and 2005). These esterase inhibitors vary structurally and act differently. In this study Part II, we demonstrated that GFJ flavonoids were carboxylestserase inhibitors. In this study, we want to further examine the mechanism of this new action of GFJ flavonoids. Ideally, the pure human esterases would be used; however, because pure human carboxyl esterases are not commercially available, the available purified porcine esterases were used as a model of the human enzyme instead. The hydrolysis of enalapril, a prodrug for the treatment of hypertension, is known to be predominantly mediated by human carboxylesterase I(hCE1), and the hydrolysis of CPT11, a carboxylic ester prodrug on the market for first line therapy of patients with advanced coloretal cancer, was dominantly mediated by human carboxyl esterase II (hCE2) (Humerickhouse et al., 2000). Therefore, CPT11 and enalapril, the selective hCEs substrates, were selected as the model compounds in this study.

\section{OBJECTIVE}

The objective of this study was to evaluate the interaction mechanism of GFJ flavonoids with esterases.

\section{METHODS}

16.1 Hydrolysis of CPT11

Chemical hydrolysis 
Studies on the chemical hydrolysis of CPT11 were performed in $0.1 \mathrm{M}$ phosphate buffer, pH 3.5, 7.0 and 7.4 respectively. CPT11 $(5 \mu \mathrm{M})$ was incubated in $0.1 \mathrm{M}$ phosphate buffer with $\mathrm{pH} 3.5,7.0$ and 7.4 , respectively. At $0,15,30$, and $60 \mathrm{~min}$, reactions were stopped by adding an equal volume of acetonitrile containing carbutamide as the internal standard. The samples were directly analyzed with LC/MS/MS for the amount of CPT11 and SN38.

\section{Hydrolysis in purified porcine esterase}

Studies on the hydrolysis of CPT11 by purified porcine esterase were performed. Purified porcine esterase $(0.1 \mathrm{unit} / \mathrm{ml})$ in $0.1 \mathrm{M}$ potassium phosphate buffer, $\mathrm{pH}$ 7.4) were incubated with CPT11 $(1.0,2.0,4.0,8.0,16.0,32 \mu \mathrm{M})$ in 96-well plates. Reactions were initiated by addition of CPT11 in $0.1 \mathrm{M}$ potassium phosphate buffer, $\mathrm{pH} 7.4$ containing $<0.2 \%$ DMSO, and were carried out for 10 minutes at $37^{\circ} \mathrm{C}$. Reactions were stopped by the addition of acetonitrile $(1: 1 . \mathrm{v}: \mathrm{v})$ containing carbutamide as the internal standard. The plates were stored at $4^{\circ} \mathrm{C}$ for 30 minutes, and then centrifuged in order to pellet the precipitated proteins. The supernatants were analyzed by LC/MS/MS for relative amounts of SN38.

Hydrolysis in human liver microsomes and S9 fractions

Studies on the hydrolysis of CPT11 by human liver S9 and microsomes were performed. Human liver microsomes and $\mathrm{S} 9(1 \mathrm{mg} / \mathrm{mL}$ in $0.1 \mathrm{M}$ potassium phosphate buffer, $\mathrm{pH} 7.4)$ were incubated with $\mathrm{CPT} 11(0,0.125,0.25,0.5,1.0$, $2.0,4.0,8.0,16.0 \mu \mathrm{M})$ in 96 -well plates. Reactions were initiated by addition of CPT11 in $0.1 \mathrm{M}$ potassium phosphate buffer, $\mathrm{pH} 7.4$ containing $<0.2 \%$ DMSO, and were carried out for 30 minutes at $37^{\circ} \mathrm{C}$. Reactions were stopped by addition of acetonitrile (1:1 v:v) containing carbutamide as the internal standard. The samples were processed and analyzed as described above. 
16.2 Hydrolysis of enalapril

\section{Chemical hydrolysis}

Studies on the chemical hydrolysis of enalapril were performed. Enalapril $(5 \mu \mathrm{M})$

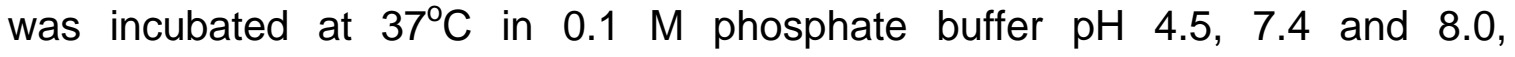
respectively. At $0,15,80$, and $240 \mathrm{~min}$, an equal volume of acetonitrile containing carbutamide as the internal standard was added into the incubation mixtures. The samples were directly analyzed with LC/MS/MS for enalapril and enalaprilat.

Hydrolysis by purified porcine esterase

Studies on the hydrolysis of enalapril by purified porcine esterase were performed. Purified porcine esterases $0.025 \mathrm{unit} / \mathrm{mL}$ in $0.1 \mathrm{M}$ potassium phosphate buffer $(\mathrm{pH} 7.4)$ were incubated with enalapril $(0,15.6,31.2,62.5,125$, 250 ) in 96-well plates. Reactions were initiated by addition of enalapril in $0.1 \mathrm{M}$ potassium phosphate buffer, $\mathrm{pH} 7.4$ containing $<0.2 \% \mathrm{DMSO}$, and were carried out for 10 minutes at $37^{\circ} \mathrm{C}$. Reactions were stopped by addition of acetonitrile $(1: 1, v: v)$ containing carbutamide as the internal standard. The samples were stored at $4^{\circ} \mathrm{C}$ for 30 minutes, and then centrifuged in order to pellet the precipitated proteins. The supernatants were diluted 10-fold using water, and then were analyzed by LC/MS/MS for relative amounts of enalaprilat.

Hydrolysis by human liver microsomes or S9 fractions

Studies on the hydrolysis of enalapril by human liver $\mathrm{S9}$ and microsomes were performed. Human liver microsomes and $S 9(1 \mathrm{mg} / \mathrm{mL}$ in $0.1 \mathrm{M}$ potassium phosphate buffer, $\mathrm{pH} 7.4)$ were incubated with enalapril $(0,15.6,31.2,62.5,125$, 250 ) in 96-well plates. Reactions were initiated by addition of Enalapril in $0.1 \mathrm{M}$ potassium phosphate buffer, $\mathrm{pH} 7.4$ containing $<0.2 \% \mathrm{DMSO}$, and were carried out for 10 minutes at $37^{\circ} \mathrm{C}$. Reactions were stopped by addition of acetonitrile 
$(1: 1, v: v)$ containing carbutamide as the internal standard. The samples were processed and analyzed as described above.

\section{Inhibition studies}

The inhibition mechanism of naringenin on hydrolysis of CPT11 by purified porcine esterase was investigated. Purified porcine esterase $(0.1 \mathrm{unit} / \mathrm{mL})$ in 0.1 $\mathrm{M}$ potassium phosphate buffer $\mathrm{pH} 7.4$ were incubated at $37^{\circ} \mathrm{C}$ with CPT11 of concentrations $(1,2,4,8,16,32 \mu \mathrm{M})$ and one of the five concentrations $(0,15.6$, $31.2,62.5,125,250)$ of naringenin. Reactions were initiated with the addition of purified porcine esterase. After $10 \mathrm{~min}$, reactions were stopped by addition of $1 \%$ acetic acid in acetonitrile (1:1, v:v) containing carbutamide as the internal standard. The sample plates were stored at $4{ }^{\circ} \mathrm{C}$ for 30 minutes, and then centrifuged in order to pellet the precipitated proteins. The supernatants were analyzed by LC/MS/MS for relative amounts of SN38.

The inhibition mechanism of kaempferol and quercetin on hydrolysis of CPT11 by purified porcine esterase was investigated. Purified porcine esterase $(0.1 \mathrm{unit} / \mathrm{mL})$ in $0.1 \mathrm{M}$ potassium phosphate buffer $\mathrm{pH} 7.4$ were incubated at $37^{\circ} \mathrm{C}$ with CPT11 of concentrations $(1,2,4,8,16,32 \mu \mathrm{M})$ and one of the five concentrations $(0$, $0.13,0.25,0.5,1,2 \mu \mathrm{M})$ of kaempferol and quercetin. Reactions were initiated with the addition of CPT11. After $10 \mathrm{~min}$, reactions were stopped by addition of acetonitrile $(1: 1, \mathrm{v}: \mathrm{v})$ containing carbutamide as the internal standard. The samples were analyzed as described above.

The inhibition mechanism of naringenin on hydrolysis of enalapril by purified porcine esterase was investigated. Purified porcine esterase $(0.025 \mathrm{unit} / \mathrm{mL})$ in $0.1 \mathrm{M}$ potassium phosphate buffer $\mathrm{pH} 7.4$ were incubated at $37^{\circ} \mathrm{C}$ with enalapril of concentrations $(5,10,25,50,100,250 \mu \mathrm{M})$ and one of the five concentrations $(0,15.6,31.3,62.5,125,250)$ of naringenin. Reactions were initiated with the addition of enalapril. After $10 \mathrm{~min}$, reactions were stopped by addition of 
acetonitrile (1:1, v:v) containing carbutamide as the internal standard. The samples were processed and analyzed as described above.

The inhibition mechanism of kaempferol and quercetin on hydrolysis of enalapril by purified porcine esterase was investigated. Purified porcine esterase $(0.025$ unit $/ \mathrm{mL}$ ) in $0.1 \mathrm{M}$ potassium phosphate buffer $\mathrm{pH} 7.4$ were incubated at $37^{\circ} \mathrm{C}$ with enalapril of concentrations $(5,10,25,50,100,250 \mu \mathrm{M})$ and one of the five concentrations $(0,0.13,0.25,0.5,1,2 \mu \mathrm{M})$ of kaempferol and quercetin. Reactions were initiated with the addition of enalapril. After $10 \mathrm{~min}$, reactions were stopped by addition of acetonitrile $(1: 1, \mathrm{v}: \mathrm{v})$ containing carbutamide as the internal standard. The samples were processed and analyzed as described above.

\section{LC/MS Analysis}

The LC/MS method for enalaprilt analysis was described in Part I and II. SN38 and CPT11 were also analyzed with an LC/MS method. The LC/MS/MS system consisted of a binary high performance liquid chromatography pump (1100; Agilent Technologies, Palo Alto, CA), an HTS PAL autosampler (LEAP Technologies, Carrboro, NC), and a triple-quadrupole mass spectrometer (APF 4000; Applied Biosystems, Foster City, CA). Separation was performed on an YMC-ODS-AQ C18 column ( $30 \mathrm{~mm} \times 2.0 \mathrm{~mm}$; Waters, Milford, MA) using mixtures of formic acid $(0.1 \%)$ in water and acetonitrile as a mobile phase. The mass spectrometer was operated in the multiple reactions monitoring mode (MRM) using positive ion electrospray ionization. MRM was set at $\mathrm{m} / \mathrm{z} 587.4$ ? 124.4 for CPT11 and 587.4 ? 124.4 for SN38. The quantification limit for CPT11 and SN38 was generally $2 \mathrm{nM}$.

Data analysis 
Inhibition mechanism. Direct plot, Lineweaver-Burk plot, and Eadie-Hofstee plot were used to evaluate inhibition mechanism.

Ki Determination. A non-simultaneous, nonlinear regression analysis was employed. Each of the six independent experimental sets (control and each of the five inhibitor concentrations) was fit individually by non-linear regression to the Michaelis-Menten expression using Prism software (GraphPad, San Diego, $\mathrm{CA}$ ). Estimates of $\mathrm{Ki}$ were obtained from the linear regression plot of $\mathrm{K}_{\mathrm{M} \text {,app }} \mathrm{V}_{\max }$ as a function of the inhibitor concentration. The method for Ki determination is described by Copeland et al., 1996.

\section{RESULTS AND DISCUSSIONS}

\subsection{Results}

CPT-11 hydrolysis by purified porcine esterase, and the effect of kaempferol, quercetin, and naringenin

Less than 0.1 percent of the total CPT11 was hydrolyzed to SN38 in phosphate buffer $\mathrm{pH} 7.4$ (Figure 42A), thus non-enzyme mediated hydrolysis was negligible. All three flavones, kaempferol, quercetin and naringenin, showed inhibitory effects on CPT11 hydrolysis by purified porcine esterases. Kaempferol showed potent inhibitory effects on CPT11 hydrolysis (SN38 formation). The inhibition observed occurred over a broad range of substrate and inhibitor concentrations. Lineweaver-Burk plot for SN38 formation revealed common intercepts at the ordinate consistent with competitive inhibition. Representative graph for kaempferol is presented in Figure 45B. The direct plot is presented in Figure 45A and the values of $V_{\max }$ and $K_{m}$ from direct plot are summarized in Table 13. Kaempferol did not affect $\mathrm{V}_{\max }$ but significantly reduce $\mathrm{K}_{\mathrm{m}}$ suggesting competitive inhibition, consistent with the Lineweaver-Burk plot. As with kaempferol, the inhibition by quercetin and naringenin occurred over a broad range of substrate 
and inhibitor concentrations. Representative graphs for quercetin and naringenin are presented in Figures $46 \mathrm{~A}$ and $47 \mathrm{~A}$ and Figures 46B, 47B, respectively, values of apparent $V_{\max }$ and $\mathrm{K}_{\mathrm{m}}$ of CPT11 are summarized in Table 13. Primary Lineweaver-Burk plots showed a common intercept at the $y$-axis. The mechanism of inhibition by both compounds is competitive. By non-simultaneous, nonlinear regression analysis using the competitive model, estimates of the mean Ki for purified porcine liver esterase (Table 15) were $0.102 \mu \mathrm{M}, 0.752 \mu \mathrm{M}$, and $55.4 \mu \mathrm{M}$ for kaempferol, quercetin, and naringenin, respectively.

Enlapril hydrolysis by purified porcine esterase, and the effect of kaempferol, quercetin, and naringenin

Less than 0.2 percent of the total enalapril was hydrolyzed to enalaprilat in phosphate buffer $\mathrm{pH} 7.4$ (Figure 42B), thus non-enzyme mediated hydrolysis was negligible. Kaempferol, quercetin, and naringenin also showed inhibitory effects on hydrolysis of enalapril by purified porcine esterase. Eadie-Hofstee plots of kaempferol, quercetin, and naringenin inhibition are presented Figures 48B, 49B, and 50B. Eadie-Hofstee plots of kaempferol, quercetin, and naringenin esterase inhibition were best described to be parallel lines suggesting that the mechanism of esterase inhibition by the three flavonids was noncompetitive inhibition. The direct plots of kaempferol, quercetin, and naringenin inhibition are presented in Figures 48A, 49A, and 50A; and the values of their apparent $V_{\max }$ and $K_{m}$ were summarized in Table 14. Kaempferol, quercetin, and naringenin did not affect $\mathrm{K}_{\mathrm{m}}$ of enalapril hydrolysis but significantly reduce the $V_{\max }$ suggesting noncompetitive inhibition, consistent with the results obtained from Eadie-Hofstee plots.

\section{2 Discussion}

Known carboxyl esterase inhibitors vary widely in size and structure, a class belonging to aromatic diones being prototypical inhibitors (Wadkins et.al, 2005). 
These compounds acted as partially competitive reversible inhibitors, with the serine of esterase binding to the carbonyl carbon of the dione via a nucleophilic attack. A series of benzene sulfonamides were identified as specific inhibitors for hCE2. As with the aromatic dione family, these compounds act as partially competitive, reversible inhibitors (Wadkins et al, 2004). Flavonoids are inhibiting phosphodiesterase (Peluso MR, 2006), however, this study demonstrated

flavonoids inhibited carboxylesterases. Three GFJ flavonoids were competitive inhibitors for CTP11 hydrolysis and noncompetitive inhibitors for enalapril hydrolysis. As shown in Table 15, kaempferol appeared to be a much more potent carboxyl esterase inhibitor than naringenin. The $K_{i}$ value is $0.1 \mu \mathrm{M}$ for kaempferol, and $55.4 \mu \mathrm{M}$ for naringenin, a >500-fold difference. However, structurally, the only difference between kaempferol and naringenin is the extra enol group in Ring-B. The enol group can undergo tautomerization to the keto form (a dione), making the carbonyl carbon more electropositive and facilitating nucleophilic attack by serine of esterase on the carbonyl carbon. It is also interesting to note that the inhibition potential of flavonoid aglycones and flavonoid glycosides is different from each other.

\section{CONCLUSIONS}

This study showed that kaempferol, quercetin, and naringenin competitively inhibited hydrolysis of CPT11 (to SN38) and noncompetitively inhibited hydrolysis of enalapril (to enalaprilat). 


\section{SUMMARY}

This report describes a newly identified potential of GFJ in mediating pharmacokinetic drug drug interactions due to its capability of esterase inhibition.

First, we demonstrate that GFJ inhibits purified porcine esterase activity towards p-nitrophenylacetate and the prodrugs lovastatin and enalapril. In rat and human hepatic or gut S9 fractions and rat gut lumen GFJ inhibited the hydrolysis of enalapril and lovastatin, which are known to be metabolized principally by esterases, with lovastatin metabolized also by CYP3A. In Caco-2 cells, with minimal CYP3A activity, permeability of these prodrugs was increased in the presence of GFJ. In rats, oral coadministration of GFJ or an esterase inhibitor bis-(p-nitrophenylphosphate) with the prodrugs led to respective increases in plasma AUC by $70 \%$ or $57 \%$ for enalaprilat and $279 \%$ or $141 \%$ for lovastatin acid. Additionally, portal vein cannulated rats pretreated with GFJ at -15 and -2 $\mathrm{hr}$ prior to lovastatin administration (10 mg/kg PO) as a solution (a) in water showed $49 \%$ increase (CYP3A inhibited), and (b) in GFJ showed 116\% increase (both CYP3A and gut esterase inhibited) in the portal plasma exposure to the active acid, compared to a non-GFJ pretreatment group. Overall, along with the CYP3A inactivation, the decreased esterase activity also played a significant role in increasing the metabolic stability and permeability of esters by GFJ leading to enhancement of exposure to the active drugs in rats. These new esterase inhibition findings indicate that the potential of DDIs between ester prodrugs and GFJ should also be considered in the clinic.

We also explored ingredients of GFJ responsible for this new GFJ-prodrug interaction. The esterase inhibitory potential of ten constitutive flavonoids and furanocoumarins were investigated towards para-nitrophenylacetate (PNPA) hydrolysis. The furanocoumarins bergamottin, 6',7'-dihydroxybergamottin and bergapten and the glycosidic flavonoids naringin and hesperidin, at concentrations found in GFJ or higher did not inhibit the hydrolysis of PNPA by 
purified porcine esterase and human liver microsomes. However, the flavonoid aglycones morin, galangin, kaempferol, quercetin, and naringenin showed appreciable inhibition of PNPA hydrolysis with $\mathrm{IC}_{50}$ values of 1.8, 2.8, 5.1, 5.9, and $110 \mu \mathrm{M}$ in purified porcine esterase, and $80,81,62,43$, and $30 \mu \mathrm{M}$ in human liver microsomes, respectively. In Caco-2 cells, demonstrated to contain minimal CYP3A activity, the permeability coefficient of prodrug lovastatin and enalapril was increased in the presence of the active flavonoids kaempferol and naringenin, consistent with inhibition of esterase activity. In rats, oral coadministration of kaempferol and naringenin with these prodrugs led to significant increases in plasma exposure to the active acids. Additionally, in portal vein cannulated rats coadministration of lovastatin with kaempferol (10 $\mathrm{mg} / \mathrm{kg}$ ) led to $154 \%$ and $113 \%$ increase in the portal plasma exposure to the prodrug and active acid, compared to coadministration with water. The contribution of CYP3A inhibition was demonstrated to be minimal. Overall, a series of flavonoids present in GFJ are identified as carboxylesterase inhibitors, of which kaempferol, and naringenin are shown to mediate pharmacokinetic drug interaction with prodrugs lovastatin and enalapril due to their capability of esterase inhibition. We also used two human esterase selective substrates, CPT11 and enalapril to investigate the interaction mechanism between GFJ flavonoids and esterase. Three GFJ flavonoids kaempferol, quercetin, and naringenin showed competitive inhibition on hydrolysis of CPT11 (to SN38) and noncompetitive inhibition of hydrolysis of enalapril (to enalaprilat). 


\section{BIBLIOGRAPHY}

Bailey DG, Spence JD, Edgar B, Bayliff CD, Arnold JM. (1989) Ethanol enhances the hemodynamic effects of felodipine. Clin Invest Med. 12:357-62.

Bailey DG, Spence JD, Munoz C, Arnold JM. (1991) Interaction of citrus juices with felodipine and nifedipine. Lancet. 337:268-9

Bailey DG, Arnold JM, Munoz C, Spence JD (1993) Grapefruit juice--felodipine interaction: mechanism, predictability, and effect of naringin. Clin Pharmacol Ther 53:637-42

Bailey DG, Kreeft JH, Munoz C, Freeman DJ, Bend JR. (1998) Grapefruit juicefelodipine interaction: effect of naringin and 6',7'-dihydroxybergamottin in humans . Clin Pharmacol Ther. 64:248-56

Bailey DG, Malcolm J, Arnold O, Spence JD. (2004) Grapefruit juice-drug interactions. Br J Clin Pharmacol. 58:S831-40

Bailey DG, Dresser GK. (2004) Interactions between grapefruit juice and cardiovascular drugs. Am J Cardiovasc Drugs. 4:281-97

Benton RE, Honig PK, Zamani K, Cantilena LR, Woosley RL. (1996) Grapefruit juice alters terfenadine pharmacokinetics, resulting in prolongation of repolarization on the electrocardiogram. Clin Pharmacol Ther. 59:383-8

Campbell CJ, Chantrell LJ, Eastmond R. (1987) Purification and partial characterization of rat intestinal cefuroxime axetil esterase. Biochem. Pharmacol. 36: 2317-2324 
Chen C, Mireles RJ, Campbell SD, Lin J, Mills JB, Xu JJ, Smolarek TA.( 2005) Differential interaction of 3-hydroxy-3-methylglutaryl-CoA reductase inhibitors with ABCB1, ABCC2, and OATP1B1. Drug Metab Dispos 33:537-46

Copeland RA (1996) Enzymes: A Practical Introduction to Structure, Mechanism, and Data Analysis pp195-197, VCH Publishers, New York.

Culm-Merdek KE, von Moltke LL, Gan L, Horan KA, Reynolds R, Harmatz JS, Court MH, Greenblatt DJ (2006) Effect of extended exposure to grapefruit juice on cytochrome P450 3A activity in humans: comparison with ritonavir. Clin Pharmacol Ther. 79:243-54

Campbell CJ, Chantrell LJ, Eastmond, R (1987) Purification and partial characterization of rat intestinal cefuroxime axetil esterase. Biochem Pharmacol. 36: 2317-2324

Danks MK, Morton CL, Krull EJ, Cheshire PJ, Richmond LB, Naeve CW, Pawlik CA, Houghton PJ, Potter PM (1999) Comparison of activation of CPT-11 by rabbit and human carboxylesterases for use in enzyme/prodrug therapy. Clin Cancer Res. 5:917-924.

De Castro WV, Mertens-Talcott S, Rubner A, Butterweck V, and Derendorf H (2006) Variation of flavonoids and furanocoumarins in grapefruit juices: A potential source of variability in grapefruit juice-drug interaction studies. J Agric Food Chem 54:249-255

Dresser GK, Bailey DG, Leake BF, Schwarz UI, Dawson PA, Freeman DJ, Kim RB. (2002)Fruit juices inhibit organic anion transporting polypeptide-mediated drug uptake to decrease the oral availability of fexofenadine. Clin Pharmacol Ther. 71:11-20 
Ducharme MP, Warbasse LH, and Edwards DJ (1995) Disposition of intravenous and oral cyclosporine after administration with grapefruit juice. Clin Pharmacol Ther. 57:485-91

Ducharme MP, Warbasse LH, and Edwards DJ (1995) Disposition of intravenous and oral cyclosporine after administration with grapefruit juice. Clin Pharmacol Ther. 57:485-91.

Drummer OH, Nicolaci J, lakovidis D (1990) Biliary excretion and conjugation of diacid angiotensin-converting enzyme inhibitors. I Pharmacol Exp Ther. 252:1202-6

Duggan DE, Chen IW, Bayne WF, Halpin RA, Duncan CA, Schwartz MS, Stubbs RJ, Vickers S. (1988) The physiological disposition of lovastatin, Drug Metab. Dispos. 17:166-173.

Edwards DJ, Bellevue FH 3rd, Woster PM (1996) Identification of 6, 7'dihydroxybergamottin, a cytochrome P450 inhibitor, in grapefruit juice. Drug Metab Dispos

24:1287-90

Fleming CD, Bencharit S, Edwards CC, Hyatt JL, Tsurkan L, Bai F, Fraga C, Morton CL, Howard-Williams EL, Potter PM, Redinbo MR, (2005) Structural insights into drug processing by human carboxylesterase 1: tamoxifen, mevastatin, and inhibition by benzil. J. Mol. Biol. 352:165-177

Fukuda K, Ohta T, Oshima Y, Ohashi N, Yoshikawa M, Yamazoe Y (1997) Specific CYP3A4 inhibitors in grapefruit juice: furocoumarin dimers as components of drug interaction. Pharmacogenetics 7:391-6 
Guo LQ, Fukuda K, Ohta T, and Yamaoe Y (2000) Role of furanocoumarin derivatives on grapefruit juice-mediated inhibition of human CYP3A4 Activity. Drug Metab Dispos 28:766-771

Halpin RA, Ulm EH, Till AE, Kari PH, Vyas KP, Hunninghake DB, Duggan DE (1993) Biotransformation of lovastatin. V. Species differences in in vivo metabolite profiles of mouse, rat, dog, and human. Drug Metabolism and Disposition 21:1003-11

He K, lyer KR, Hayes RN, Sinz MW, Woolf TF, Hollenberg PF(1998) Inactivation of cytochrome P450 3A4 by bergamottin, a component of grapefruit juice. Chem Res Toxicol. 11:252-9

Ho PC, Saville DJ, Coville PF, Wanwimolruk S (2000) Content of CYP3A4 inhibitors, naringin, naringenin and bergapten in grapefruit and grapefruit juice products. Pharm Acta Helv 74:379-85

Ho PC, Saville DJ, Wanwimolruk S (2001) Inhibition of human CYP3A4 activity by grapefruit flavonoids, furanocoumarins and related compounds. J Pharm Sci. 4:217-27

Honda Y, Ushigome F, Koyabu N, Morimoto S, Shoyama Y, Uchiumi T, Kuwano M, Ohtani H, Sawada Y (2004) Effects of grapefruit juice and orange juice components on P-glycoprotein- and MRP2-mediated drug efflux. Br J Pharmacol. 143:856-64

Humerickhouse R, Lohrbach K, Li L, Bosron WF, Dolan ME (2000) Characterization of CPT-11 hydrolysis by human liver carboxylesterase isoforms hCE-1 and hCE-2. Cancer Res. 60:1189-92. 
Jignesh P, Balasubrahmanyam B, Surajit D, Mitra D, and Ashim K (2004) In Vitro interaction of the HIV protease inhibitor ritonavir with herbal constituents: changes in P-gp and CYP3A4 activity. Am J Therapeut 11:262-277

Kupferschmidt HH, Ha HR, Ziegler WH, Meier PJ, Krahenbuhl S (1995) Interaction between grapefruit juice and midazolam in humans. Clin Pharmacol Ther. 58:20-8.

Kantola T, Kivisto KT, Neuvonen PJ (1998) Grapefruit juice greatly increases serum concentrations of lovastatin and lovastatin acid. Clin Pharmacol Ther. 63:397-402.

Kim H, Yoon YJ, Shon JH, Cha IJ, Shin JG, Liu KH (2006) Inhibitory effects of fruit juices on CYP3A activity. Drug Metab Dispos. 34:521-3.

Kitagawa S, Takeda J, and Sato S (1999) Uptake of Enalapril by rabbit small intestinal Brush-Border Membrance vesicles. Biol Pharm Bull. 22:762-764

Liu L, Cui Y, Chung AY, Shitara Y, Sugiyama Y, Keppler D, Pang KS (2006) Vectorial transport of enalapril by Oatp1a1/Mrp2 and OATP1B1 and OATP1B3/MRP2 in rat and human livers. J Pharmacol Exp Ther. 318:395-402.

Morrison RA, Chong S, Marino AM, Wasserman MA, Timmins P, Moore VA, Irwin WJ. (1996) Suitability of enalapril as a probe of the dipeptide transporter system: in vitro and in vivo studies. Pharm Res. 13:1078-82

Pang KS, Wang PJ, Chung AY, Wolkoff AW (1998) The modified dipeptide, enalapril, an angiotensin-converting enzyme inhibitor, is transported by the rat liver organic anion transport protein, Hepatology 28:1341-6. 
Peluso MR. (2006) Flavonoids attenuate cardiovascular disease, inhibit phosphodiesterase, and modulate lipid homeostasis in adipose tissue and liver. Exp Biol Med. 231:1287-99

Pindel EV, Kedishvili NY, Abraham TL, Brzezinski MR, Zhang J, Dean RA, Bosron WF (1997) Purification and cloning of a broad substrate specificity human liver carboxylesterase that catalyzes the hydrolysis of cocaine and heroin. $\mathrm{J} B i o l$ Chem. 272:14769-14775

Potter PM, Pawlik CA, Morton CL, Naeve CW, and Danks MK (1998) Isolation and partial characterization of a cDNA encoding a rabbit liver carboxylesterase that activates the prodrug irinotecan (CPT-11). Cancer Res. 58: 2646-2651

Rashid J, McKinstry C, Renwick AG, Dimhuber M, Waller DG, George CF (1993) Quercetin, an in vitro inhibitor of CYP3A, does not contribute to the interaction between nifedipine and grapefruit juice. Br J Clin Pharmacol. 36:460-3.

Saito M, Hirata-Koizumi M, Matsumoto M, Urano T, Hasegawa R (2005) Undesirable effects of citrus juice on the pharmacokinetics of drugs: focus on recent studies. Drug Saf. 28:677-94.

Ross SA, Ziska DS, Zhao K, Elsohly MA (2000) Variance of common flavonoids by brand of grapefruit juice. Fitoterapia 71:154-61

Satoh T. and Hosokawa M (1998) The mammalian carboxylesterases from molecules to functions. Annu Rev Pharmacol Toxicol. 38:257-88

Schmiedlin-Ren P, Edwards DJ, Fitzsimmons ME, He K, Lown KS, Woster PM, Rahman A, Thummel KE, Fisher JM, Hollenberg PF, Watkins PB (1997) Mechanisms of enhanced oral availability of CYP3A4 substrates by grapefruit 
constituents. Decreased enterocyte CYP3A4 concentration and mechanismbased inactivation by furanocoumarins. Drug Metab Dispos 25:1228-33.

Tocco DJ, deLuna FA, Duncan AE, Vassil TC, Ulm EH (1982) The physiological disposition and metabolism of enalapril maleate in laboratory animals. Drug Metab Dispos. 10:15-9

Vree TB, Dammers E, Ulc I, Horkovics-Kovats S, Ryska M, Merkx I. (2003) Differences between lovastatin and simvastatin hydrolysis in healthy male and female volunteers:gut hydrolysis of lovastatin is twice that of simvastatin. Scientific World J. 11:1332-43

Van Gelder J, Annaert P, Naesens L, De Clercq E, Van den Mooter G, Kinget R, Augustijns $P$ (1999) Inhibition of intestinal metabolism of the antiviral ester prodrug bis(POC)-PMPA by nature-identical fruit extracts as a strategy to enhance its oral absorption: an in vitro study. Pharmaceutical Research 16:10351040

Van Gelder J, Deferme S, Annaert P, Naesens L, De Clercq E, Van den Mooter G, Kinget R, Augustijns P (2000) Increased absorption of the antiviral ester prodrug tenofovir disoproxil in rat ileum by inhibiting its intestinal metabolism. Drug Metab Dispos. 28:1394-6

Van Gelder J, Deferme S, Annaert P, Naesens L, De Clercq E, Van den Mooter G, Kinget R, Augustijns $\mathrm{P}(2002)$ Intestinal absorption enhancement of the ester prodrug tenofovir disoproxil fumarate through modulation of the biochemical barrier by defined ester mixtures. Drug Metab Dispos. 30: 924-930

Vyas KP, Kari PH, Prakash SR, Duggan DE (1990) Biotransformation of lovastatin II. In vitro metabolism by rat and mouse liver microsomes and 
involvement of cytochrome P-450 in dehydrogenation of lovastatin. Drug Metab Dispos. 18:218-22

Wadkins RM, Hyatt JL, Yoon KJ, Morton CL, Lee RE, Damodaran K, Beroza P, Danks MK, Potter PM (2004) Discovery of novel selective inhibitors of human intestinal carboxylesterase for the amelioration of irinotecan-induced diarrhea: synthesis, quantitative structure-activity relationship analysis, and biological activity. Mol Pharmacol 65:1336-43

Wadkins RM, Hyatt JL, Wei X, Yoon KJ, Wierdl M Edwards CC, Morton CL, Obenauer JC, Damodaran K, Beroza P, Danks MK, Potter PM (2005) Identification and characterization of novel benzil (diphenylethane-1,2-dione) analogues as inhibitors of mammalian carboxylesterases. J Med Chem 48:2906-15

Walker CH and Mackness MI (1983) Esterase: problems of identification and classification. Biochem. Pharmacol. 32:3265-3269

Williams C. F. M. (1985) Clinical significance of esterases in man. Clin. Pharmacokin. 10: 392-403

Xia CQ, Liu N, Yang D, Miwa G, Gan LS (2005) Expression, localization, and functional characteristics of breast cancer resistance protein in Caco-2 cells. Drug Metab Dispos. 33:637-43.

Zhang J, Burnell JC, Dumaual N, Bosron WF (1999) Binding and hydrolysis of meperidine by human liver carboxylesterase hCE-1. J Pharmacol Exp.Ther. 290: 314-318.

Zhou S., Lim LY, and Chowbay B (2004) Herbal modulation of P-glycoprotein. Drug Metab Rev. 36:57-104. 


\section{TABLES}

Table1. Percentage remaining of lovastatin in pulp-free GFJ supernatant after the centrifugation of lovastatin $(20 \mu \mathrm{M})$ solutions containing various concentrations of GFJ

\begin{tabular}{|c|c|}
\hline GFJ \% & $\begin{array}{c}\text { \% lovastatin in pulp free GFJ } \\
\text { supernatant }\end{array}$ \\
\hline 0 & 100 \\
\hline 6.25 & 92.6 \\
\hline 12.5 & 87.8 \\
\hline 25 & 74.1 \\
\hline 50 & 68.2 \\
\hline 100 & 64.2 \\
\hline
\end{tabular}


Table2. Effect of the esterase inhibitor BNPP (25 mg/kg PO) on the PK parameters of lovastatin acid and enalaprilat following oral administration of lovastatin and enalapril, respectively, $(10 \mathrm{mg} / \mathrm{kg})$ to rats

\begin{tabular}{|c|ccc|ccc|}
\hline & \multicolumn{3}{|c|}{ Enalaprilat } & \multicolumn{3}{c|}{ Lovastatin acid } \\
\hline Co-administration & $\mathrm{AUC}_{0-24 \mathrm{hr}}$ & $\mathrm{T}_{\max }$ & $\mathrm{C}_{\max }$ & $\mathrm{AUC}_{0-24 \mathrm{hr}}$ & $\mathrm{T}_{\max }$ & $\mathrm{C}_{\max }$ \\
& $(\mathrm{nM} . \mathrm{hr})$ & $(\mathrm{hr})$ & $(\mathrm{nM})$ & $(\mathrm{nM} . \mathrm{hr})$ & $(\mathrm{hr})$ & $(\mathrm{nM})$ \\
\hline Water & $5743 \pm 303$ & $1.0 \pm 0.0$ & $2543 \pm 255$ & $45.3 \pm 6.20$ & $1.3 \pm 0.6$ & $10.2 \pm 1.25$ \\
BNPP & $9006 \pm 1292$ & $1.0 \pm 0.0$ & $3963 \pm 1410$ & $109 \pm 12.5$ & $1.0 \pm 0.0$ & $34.2 \pm 11.6$
\end{tabular}

Data are mean values $\pm \operatorname{stdev}(\mathrm{n}=3)$ 
Table 3. Effect of GFJ (10 mL/kg) on PK parameters of lovastatin acid and enalaprilat following oral administration of lovastatin ( $10 \mathrm{mg} / \mathrm{kg}$ ) and enalapril (10 mg/kg) to rats

\begin{tabular}{c|ccc|ccc}
\hline & \multicolumn{3}{|c|}{ Enalaprilat } & \multicolumn{3}{|c}{ Lovastatin acid } \\
\hline Co-administration & $\mathrm{AUC}_{0-24 \mathrm{hr}}$ & $\mathrm{T}_{\max }$ & $\mathrm{C}_{\max }$ & $\mathrm{AUC}_{0-24 \mathrm{hr}}$ & $\mathrm{T}_{\max }$ & $\mathrm{C}_{\max }$ \\
& $(\mathrm{nM} . \mathrm{hr})$ & $(\mathrm{hr})$ & $(\mathrm{nM})$ & $(\mathrm{nM} . \mathrm{hr})$ & $(\mathrm{hr})$ & $(\mathrm{nM})$ \\
\hline $\mathrm{H}_{2} \mathrm{O}(\mathrm{pH} 3.5)$ & $5743 \pm 303$ & $1.0 \pm 0.0$ & $2543 \pm 255$ & $57.5 \pm 24.1$ & $3.3 \pm 1.2$ & $14.8 \pm 3.3$ \\
GFJ, 1:3 diluted & $9465 \pm 1886$ & $1.0 \pm 0.0$ & $4063 \pm 635$ & $218 \pm 140$ & $2.3 \pm 1.5$ & $61.0 \pm 41.4$ \\
GFJ, 1:2 diluted & $9743 \pm 1560$ & $1.0 \pm 0.0$ & $3790 \pm 719$ & $148 \pm 56.3$ & $4.0 \pm 0.0$ & $34.8 \pm 12.6$ \\
GFJ, Concentrate & $6643 \pm 1949$ & $4.0 \pm 0.0$ & $1443 \pm 468$ & $155 \pm 38.0$ & $4.0 \pm 2.0$ & $38.0 \pm 16.1$ \\
\hline
\end{tabular}

Data are mean values $\pm \operatorname{stdev}(n=3)$ 
Table 4. Portal plasma PK parameters of lovastatin acid and lovastatin + lovastatin acid following oral coadministration of lovastatin $(10 \mathrm{mg} / \mathrm{kg}$ ) with water $(\mathrm{pH} 3.5)$ or GFJ to portal vein-cannulated rats, pretreated at -15 and $-2 \mathrm{hr}$ with water $(\mathrm{pH} 3.5)$ or GFJ

\begin{tabular}{|c|c|c|c|c|c|c|c|c|}
\hline \multirow[b]{2}{*}{ Group } & & & \multicolumn{3}{|c|}{ Lovastatin acid } & \multicolumn{3}{|c|}{ Lovastatin acid + Lovastatin } \\
\hline & $\begin{array}{l}\text { Pretreatment } \\
-2 \text { and }-15 \mathrm{~h}\end{array}$ & Co-admi. & $\begin{array}{l}\mathrm{AUC}_{0-8 \mathrm{hr}} \\
\text { (nM.hr) }\end{array}$ & $\begin{array}{l}T_{\max } \\
(\mathrm{hr})\end{array}$ & $\begin{array}{l}\mathrm{C}_{\max } \\
(\mathrm{nM})\end{array}$ & $\begin{array}{l}\mathrm{AUC}_{0-8 \mathrm{hr}} \\
\text { (nM.hr) }\end{array}$ & $\begin{array}{l}\mathrm{T}_{\max } \\
(\mathrm{hr})\end{array}$ & $\begin{array}{l}\mathrm{C}_{\max } \\
(\mathrm{nM})\end{array}$ \\
\hline$A$ & $\mathrm{H}_{2} \mathrm{O} \quad(\mathrm{pH} 3.5)$ & $\mathrm{H}_{2} \mathrm{O}$ & $1949 \pm 414$ & $2.4 \pm 1.9$ & $611 \pm 122$ & $2678 \pm 515$ & $2.4 \pm 1.9$ & $779 \pm 140$ \\
\hline$B$ & GFJ & $\mathrm{H}_{2} \mathrm{O}(\mathrm{pH} 3.5)$ & $2897 \pm 552$ & $2.5 \pm 1.0$ & $792 \pm 115$ & $3265 \pm 592$ & $2.5 \pm 1.0$ & $944 \pm 196$ \\
\hline $\mathrm{C}$ & GFJ & GFJ & $4209 \pm 883$ & $2.8 \pm 2.0$ & $1139 \pm 187$ & $4880 \pm 884$ & $2.8 \pm 2.0$ & $1306 \pm 149$ \\
\hline
\end{tabular}

Data are mean values $\pm \operatorname{stdev}(n=3)$ 
Table 5. Portal plasma PK parameters of 6'ß-hydroxylovastatin and its hydroxy acid (6'B-hydroxylovastatin acid) following oral administration of lovastatin (10 mg/kg) with water $(\mathrm{pH} 3.5)$ or GFJ to portal vein-cannulated rats, pretreated at -15 and $-2 \mathrm{hr}$ with water(pH 3.5) or GFJ

\begin{tabular}{|c|c|c|c|c|c|c|c|c|}
\hline \multirow{3}{*}{ Group } & \multirow{3}{*}{$\begin{array}{l}\text { Pretreatment } \\
-2 \text { and }-15 \mathrm{~h}\end{array}$} & \multirow{3}{*}{$\begin{array}{c}\text { Co- } \\
\text { administration }\end{array}$} & \multicolumn{3}{|c|}{ 6’ß-Hydroxylovastatin } & \multicolumn{3}{|c|}{ 6’ß-Hydroxylovastatin acid } \\
\hline & & & $A \cup C_{0-8 h r}$ & $T_{\max }$ & & $A \cup C_{0-8 h r}$ & $T_{\max }$ & \\
\hline & & & (nM.hr) & (hr) & $(\mathrm{nM})$ & (nM.hr) & (hr) & $(\mathrm{nM})$ \\
\hline$A$ & $\mathrm{H}_{2} \mathrm{O}, \mathrm{pH} 3.5$ & $\mathrm{H}_{2} \mathrm{O}, \mathrm{pH} 3.5$ & $1555 \pm 290$ & $4.1 \pm 2.6$ & $448 \pm 118$ & $740 \pm 125$ & $3.1 \pm 2.4$ & $219 \pm 65.6$ \\
\hline B & GFJ & $\mathrm{H}_{2} \mathrm{O}, \mathrm{pH} 3.5$ & $186 \pm 148$ & $1.8 \pm 1.7$ & $94.1 \pm 34.2$ & $83.1 \pm 22.1$ & $2.4 \pm 1.9$ & $51.3 \pm 21.2$ \\
\hline C & GFJ & GFJ & $181 \pm 62.3$ & $2.3 \pm 1.5$ & $114 \pm 63.2$ & $77.0 \pm 4.9$ & $2.3 \pm 1.5$ & $41.9 \pm 13.4$ \\
\hline
\end{tabular}

Data are mean values $\pm \operatorname{stdev}(n=3)$ 
Table 6. Effect of oral pretreatment with GFJ on PK parameters of lovastatin and lovastatin acid following IV administration of lovastatin $(2 \mathrm{mg} / \mathrm{kg})$ to rats

\begin{tabular}{|c|c|c|c|c|c|c|c|c|}
\hline Pretreatment & \multicolumn{4}{|c|}{ Lovastatin acid } & \multicolumn{5}{c|}{ Lovastatin } \\
\hline$(\mathrm{PO})$ & $\mathrm{AUC}_{0-2 \mathrm{hr}}$ & $\mathrm{CL}_{\mathrm{p}}$ & $\mathrm{Vd}_{\mathrm{ss}}$ & $\mathrm{t}_{1 / 2}$ & $\mathrm{AUC}_{0-2 \mathrm{hr}}$ & $\mathrm{CL}_{\mathrm{p}}$ & $\mathrm{Vd}_{\mathrm{ss}}$ & $\mathrm{t}_{1 / 2}$ \\
$-0.5 \mathrm{hr}$ & $(\mathrm{nM} . \mathrm{hr})$ & $(\mathrm{L} / \mathrm{hr} / \mathrm{kg})$ & $\mathrm{L} / \mathrm{kg}$ & $(\mathrm{hr})$ & $(\mathrm{nM} . \mathrm{hr})$ & $(\mathrm{L} / \mathrm{hr} / \mathrm{kg})$ & $\mathrm{L} / \mathrm{kg}$ & $(\mathrm{hr})$ \\
\hline Water, pH 3.5 & $840 \pm 154$ & $5.52 \pm 1.02$ & $3.61 \pm 1.03$ & $0.43 \pm 0.06$ & $131 \pm 13.0$ & $37.7 \pm 3.95$ & $12.5 \pm 2.98$ & $0.26 \pm 0.01$ \\
GFJ, 1:3 diluted & $722 \pm 103$ & $6.38 \pm 0.97$ & $4.04 \pm 0.40$ & $0.43 \pm 0.01$ & $108 \pm 11.3$ & $45.9 \pm 4.64$ & $15.4 \pm 2.37$ & $0.30 \pm 0.02$ \\
\hline
\end{tabular}

Data are mean values $\pm \operatorname{stdev}(n=3)$ 
Table 7. Effect of oral pretreatment with GFJ on PK parameters of enalapril and enalaprilat following IV administration of enalapril $(2 \mathrm{mg} / \mathrm{kg})$ to rats

\begin{tabular}{|c|c|c|c|c|c|c|c|c|}
\hline Pretreatment & \multicolumn{4}{|c|}{ Enalaprilat } & \multicolumn{4}{c|}{ Enalapril } \\
\hline$(\mathrm{PO})$ & $\mathrm{AUC}_{0-24 \mathrm{hr}}$ & $\mathrm{CL}_{\mathrm{p}}$ & $\mathrm{Vd}_{\mathrm{ss}}$ & $\mathrm{t}_{1 / 2}$ & $\mathrm{AUC}_{0-1 \mathrm{hr}}$ & $\mathrm{CL}_{\mathrm{p}}$ & $\mathrm{Vd}_{\mathrm{ss}}$ & $\mathrm{t}_{1 / 2}$ \\
$-0.5 \mathrm{hr}$ & $(\mathrm{nM} . \mathrm{hr})$ & $(\mathrm{L} / \mathrm{hr} / \mathrm{kg})$ & $\mathrm{L} / \mathrm{kg}$ & $(\mathrm{hr})$ & $(\mathrm{nM} . \mathrm{hr})$ & $(\mathrm{L} / \mathrm{hr} / \mathrm{kg})$ & $\mathrm{L} / \mathrm{kg}$ & $(\mathrm{hr})$ \\
\hline Water, pH 3.5 & $2730 \pm 486$ & $2.00 \pm 0.31$ & $3.15 \pm 1.14$ & $9.7 \pm 1.1$ & $152 \pm 29.0$ & $38.8 \pm 8.18$ & $3.95 \pm 0.76$ & $0.10 \pm 0$ \\
GFJ, 1:3 diluted & $2680 \pm 428$ & $2.00 \pm 0.32$ & $3.06 \pm 0.70$ & $8.4 \pm 1.9$ & $144 \pm 23.3$ & $40.7 \pm 7.23$ & $4.14 \pm 0.53$ & $0.10 \pm 0$ \\
\hline
\end{tabular}

Data are mean values $\pm \operatorname{stdev}(n=3)$ 
Table 8. Inhibitory effect of GFJ chemical components toward PNPA hydrolysis in purified porcine esterases

\begin{tabular}{|c|c|c|c|c|}
\hline \multirow{2}{*}{ Inhibitors } & \multicolumn{3}{|c|}{ Inhibition of PNPA Hydrolysis (\%) } & \multirow{2}{*}{$\mathrm{I}_{50}(\mu \mathrm{M})$} \\
\hline Conc. & $5(\mu \mathrm{M})$ & $50(\mu \mathrm{M})$ & $1000(\mu \mathrm{M})$ & \\
\hline Kaempferol & 50 & 80 & na & 5.1 \\
\hline Quercetin & 50 & 82 & na & 5.9 \\
\hline Morin & 60 & 90 & na & 1.8 \\
\hline Galangin & 58 & 85 & na & 2.8 \\
\hline Naringenin & $<5$ & 27 & na & 110 \\
\hline Hesperidin & $<5$ & $<5$ & na & na \\
\hline Naringin & $<5$ & $<5$ & 25 & na \\
\hline Bergamottin & $<5$ & 23 & na & na \\
\hline 6',7'-DHB & $<5$ & $<5$ & na & na \\
\hline Bergapten & $<5$ & $<5$ & na & na \\
\hline
\end{tabular}


Table 9. Inhibitory effect of GFJ chemical components toward PNPA hydrolysis in human liver microsomes

\begin{tabular}{|c|c|c|c|c|}
\hline \multirow{2}{*}{ Inhibitors } & \multicolumn{3}{|c|}{ Inhibition of PNPA Hydrolysis $(\%)$} & \multirow{2}{*}{$\mathrm{I}_{50}(\mu \mathrm{M})$} \\
\hline Conc. & $5(\mu \mathrm{M})$ & $50(\mu \mathrm{M})$ & $1000(\mu \mathrm{M})$ & \\
\hline Kaempferol & $<5$ & 45 & na & 62 \\
\hline Quercetin & $<5$ & 50 & na & 43 \\
\hline Morin & $<5$ & 40 & na & 80 \\
\hline Galangin & $<5$ & 40 & na & 81 \\
\hline Naringenin & $<5$ & 65 & na & 30 \\
\hline Hesperidin & $<5$ & $<5$ & na & na \\
\hline Naringin & $<5$ & $<5$ & $<5$ & na \\
\hline Bergamottin & $<5$ & 20 & na & na \\
\hline 6',7'-DHB & $<5$ & 10 & na & na \\
\hline Bergapten & $<5$ & $<5$ & na & na \\
\hline
\end{tabular}


Table 10. Pharmacokinetic parameters of enalaprilat following oral administration of enalapril $(10 \mathrm{mg} / \mathrm{kg}$ ) to rats and the effect of co-administrated naringenin (2 and $10 \mathrm{mg} / \mathrm{kg})$ and kaempferol (2 and $10 \mathrm{mg} / \mathrm{kg}$ )

\begin{tabular}{|c|c|c|c|}
\hline \multirow[b]{2}{*}{ Co-admin } & \multicolumn{3}{|c|}{ Enalaprilat } \\
\hline & $\begin{array}{c}\mathrm{AUC}_{0-8 \mathrm{hr}} \\
\text { (nM.hr) }\end{array}$ & $\begin{array}{c}\text { Tmax } \\
\text { (hr) }\end{array}$ & $\begin{array}{c}\mathrm{C}_{\max } \\
(\mathrm{nM})\end{array}$ \\
\hline Water & $5494 \pm 2309$ & $0.66 \pm 0.28$ & $2163 \pm 932$ \\
\hline Naringenin, 2 mg/kg & $6473 \pm 3162$ & $0.83 \pm 0.29$ & $2213 \pm 882$ \\
\hline Naringenin, $10 \mathrm{mg} / \mathrm{kg}$ & $7597 \pm 2190$ & $1.0 \pm 0.0$ & $2620 \pm 436$ \\
\hline Kaempferol, 2 mg/kg & $8814 \pm 3279$ & $0.5 \pm 0.0$ & $3033 \pm 1161$ \\
\hline Kaempferol, 10 mg/kg & $11498 \pm 3386$ & $0.8 \pm 0.2$ & $4830 \pm 1939$ \\
\hline
\end{tabular}

Data are mean values $\pm \operatorname{stdev}(n=3)$ 
Table 11. Pharmacokinetic parameters of lovastatin acid following oral administration of lovastatin (10 mg/kg) to rats and the e ffect of co-administrated naringenin (2 and $10 \mathrm{mg} / \mathrm{kg}$ ) and kaempferol (2 and $10 \mathrm{mg} / \mathrm{kg}$ )

\begin{tabular}{|c|ccc|}
\hline & \multicolumn{3}{|c|}{ Lovastatin Acid } \\
\hline Co-admin & AUC $_{0-8 \mathrm{hr}}$ & $\mathrm{T}_{\max }$ & $\mathrm{C}_{\text {max }}$ \\
$(\mathrm{nM} . \mathrm{hr})$ & $(\mathrm{hr})$ & $(\mathrm{nM})$ \\
\hline Water & $32.5 \pm 1.1$ & $0.4 \pm 0.2$ & $28.5 \pm 12.7$ \\
\hline Naringenin , $2 \mathrm{mg} / \mathrm{kg}$ & $84.1 \pm 11.7$ & $1.7 \pm 0.6$ & $38.0 \pm 6.9$ \\
Naringenin ,10 mg/kg & $126 \pm 31.2$ & $1.3 \pm 0.6$ & $48.9 \pm 8.2$ \\
\hline Kaempferol, $2 \mathrm{mg} / \mathrm{kg}$ & $88.1 \pm 16.5$ & $1.6 \pm 0.5$ & $41.6 \pm 5.2$ \\
Kaempferol,10 mg/kg & $112 \pm 11.1$ & $0.4 \pm 0.14$ & $72.0 \pm 42.9$
\end{tabular}

Data are mean values $\pm \operatorname{stdev}(n=3)$ 
Table12. Pharmacokinetic parameters of lovastatin, lovastatin acid, and 6'ß-hydroxylovastatin in portal vein following oral administration of lovastatin $(10 \mathrm{mg} / \mathrm{kg}$ ) with water and kaempferol,10 mg/kg

\begin{tabular}{|c|c|c|c|c|c|c|}
\hline \multirow{2}{*}{ Co-administration } & \multicolumn{3}{|c|}{ Water } & \multicolumn{3}{c|}{ Kaempferol, $10 \mathrm{mg} / \mathrm{kg}$} \\
\hline & $\mathrm{AUC}$ 0-8 hr & $\mathrm{T}_{\max }$ & $\mathrm{C}_{\max }$ & $\mathrm{AUC}_{0-8 \mathrm{hr}}$ & $\mathrm{T}_{\max }$ & $\mathrm{C}_{\max }$ \\
\hline & $(\mathrm{nM} . \mathrm{hr})$ & $(\mathrm{hr})$ & $(\mathrm{nM})$ & $(\mathrm{nM})$ & $(\mathrm{hr})$ & $(\mathrm{nM})$ \\
\hline Lovastatin & $1440 \pm 678$ & $2.3 \pm 0.7$ & $358 \pm 67$ & $3662 \pm 990^{*}$ & $2.4 \pm 2.0$ & $985 \pm 410$ \\
\hline $\begin{array}{c}\text { Lovastatin Acid } \\
\begin{array}{c}\text { 6'ß- } \\
\text { Hydroxylovastatin }\end{array}\end{array}$ & $3960 \pm 482$ & $2.1 \pm 2.5$ & $1042 \pm 191$ & $8420 \pm 2698^{*}$ & $2.5 \pm 1.7$ & $2425 \pm 946$ \\
\hline
\end{tabular}

Data are mean values $\pm \operatorname{stdev}(n=3)$ 
Table13. Parameters of Michaelis-Menten kinetics of CPT11 by purified porcine esterases, and the effect of naringenin, kaempferol, and quercetin

\begin{tabular}{|c|c|c|c|c|c|c|}
\hline \multirow{2}{*}{ CPT11 } & \multicolumn{6}{|c|}{ Nargingenin $(\mu \mathrm{M})$} \\
\hline & 0 & 15.6 & 31.2 & 62.5 & 125 & 250 \\
\hline $\begin{array}{c}\text { Vmax } \\
\text { (pmol/unit/min) }\end{array}$ & $0.56 \pm 0.024$ & na & $0.56 \pm 0.024$ & $0.57 \pm 0.024$ & $0.58 \pm 0.018$ & $0.70 \pm 0.051$ \\
\hline \multirow[t]{3}{*}{$\mathrm{Km}(\mu \mathrm{M})$} & $4.8 \pm 0.64$ & na & $8.8 \pm 0.83$ & $12 \pm 1.2$ & $19 \pm 1.2$ & $37 \pm 4.3$ \\
\hline & \multicolumn{6}{|c|}{ Kaempferol $(\mu \mathrm{M})$} \\
\hline & 0 & 0.13 & 0.25 & 0.5 & 1.0 & 2.0 \\
\hline $\begin{array}{c}\text { Vmax } \\
\text { (pmol/unit/min) }\end{array}$ & $0.60 \pm 0.022$ & $0.55 \pm 0.038$ & $0.56 \pm 0.044$ & $0.52 \pm 0.036$ & $0.55 \pm 0.025$ & $1.3 \pm 0.90$ \\
\hline \multirow{3}{*}{$\mathrm{Km}(\mu \mathrm{M})$} & $5.2 \pm 0.58$ & $7.2 \pm 1.3$ & $11 \pm 2.0$ & $15 \pm 2.3$ & $30 \pm 2.3$ & $146 \pm 124$ \\
\hline & \multicolumn{6}{|c|}{ Quercetin $(\mu \mathrm{M})$} \\
\hline & 0 & 0.13 & 0.25 & 0.5 & 1.0 & 2.0 \\
\hline $\begin{array}{c}\text { Vmax } \\
\text { (pmol/unit/min) }\end{array}$ & $0.59 \pm 0.012$ & $0.65 \pm 0.024$ & $0.61 \pm 0.023$ & $0.56 \pm 0.047$ & $0.67 \pm 0.066$ & $0.80 \pm 0.10$ \\
\hline $\mathrm{Km}(\mu \mathrm{M})$ & $4.7 \pm 0.29$ & $7.8 \pm 0.78$ & $7.5 \pm 0.76$ & $7.5 \pm 1.6$ & $14 \pm 3.0$ & $25 \pm 5.7$ \\
\hline
\end{tabular}


Table14. Parameters of Michaelis-Menten kinetics of enalapril hydrolysis by purified porcine esterases, and the effect of naringenin, kaempferol, and quercetin

\begin{tabular}{|c|c|c|c|c|c|c|}
\hline \multirow{2}{*}{ Enalapril } & \multicolumn{6}{|c|}{ Nargingenin $(\mu \mathrm{M})$} \\
\hline & 0 & 15.6 & 31.2 & 62.5 & 125 & 250 \\
\hline $\begin{array}{c}\text { Vmax } \\
\text { (pmol/unit/min) }\end{array}$ & $38.7 \pm 5.07$ & $38.2 \pm 8.58$ & $34.1 \pm 2.11$ & $40.3 \pm 9.74$ & $32.8 \pm 4.43$ & $22.6 \pm 3.10$ \\
\hline \multirow[t]{3}{*}{$\mathrm{Km}(\mu \mathrm{M})$} & $266 \pm 56.9$ & $313 \pm 109$ & $212 \pm 23.0$ & $361 \pm 130$ & $284 \pm 61.5$ & $119 \pm 34.3$ \\
\hline & \multicolumn{6}{|c|}{ Kaempferol $(\mu \mathrm{M})$} \\
\hline & 0 & 0.13 & 0.25 & 0.5 & 1.0 & 2.0 \\
\hline $\begin{array}{c}\text { Vmax } \\
\text { (pmol/unit/min) }\end{array}$ & $38.7 \pm 5.07$ & $33.9 \pm 5.02$ & $23.5 \pm 2.13$ & $25.2 \pm 3.44$ & $22.0 \pm 2.14$ & $19.6 \pm 3.30$ \\
\hline \multirow[t]{2}{*}{$\mathrm{Km}(\mu \mathrm{M})$} & $266 \pm 56.9$ & $277 \pm 66.2$ & $201 \pm 32.5$ & $319 \pm 67.3$ & $447 \pm 61.6$ & $558 \pm 126$ \\
\hline & 0 & 0.13 & 0.25 & 0.5 & 1.0 & 2.0 \\
\hline $\begin{array}{c}\text { Vmax } \\
\text { (pmol/unit/min) }\end{array}$ & $47.1 \pm 9.20$ & $44.6 \pm 9.71$ & $50.1 \pm 17.0$ & $44.6 \pm 7.59$ & $25.3 \pm 5.18$ & $23.8 \pm 4.05$ \\
\hline $\mathrm{Km}(\mu \mathrm{M})$ & $291 \pm 90.3$ & $300 \pm 103$ & $435 \pm 210$ & $400 \pm 99.0$ & $290 \pm 94.1$ & $317 \pm 83.6$ \\
\hline
\end{tabular}


Table15. Inhibition constants of kaempferol, quercetin, and naringenin on CPT11 and enalapril hydrolysis by purified porcine esterases

\begin{tabular}{|c|c|c|c|}
\hline & & \multicolumn{2}{|c|}{ Inhibition Constants, $\mathrm{K}_{\mathrm{i}}(\mu \mathrm{M})$} \\
\hline & & $\begin{array}{c}\text { CPT11 } \\
\text { Hydrolysis } \\
\end{array}$ & $\begin{array}{c}\text { Enalapril } \\
\text { Hydrolysis } \\
\end{array}$ \\
\hline Kaempferol & & 0.102 & 0.442 \\
\hline Quercetin & & 0.752 & 1.23 \\
\hline Naringenin & & 55.40 & 89.6 \\
\hline
\end{tabular}




\section{FIGURES}

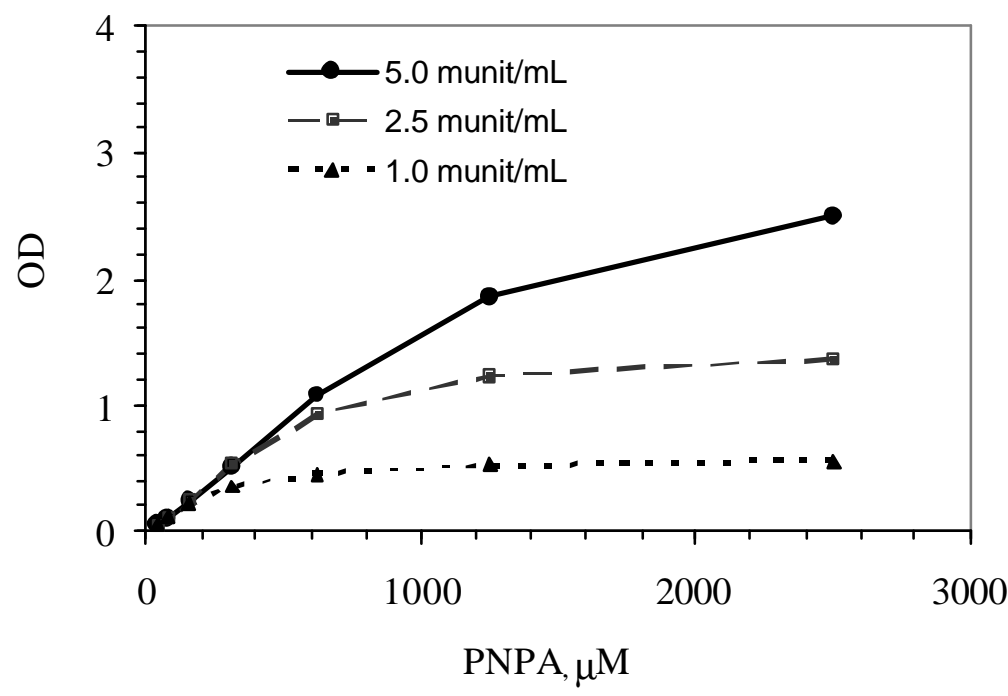

Figure 1. PNPA hydrolysis by purified porcine esterases 


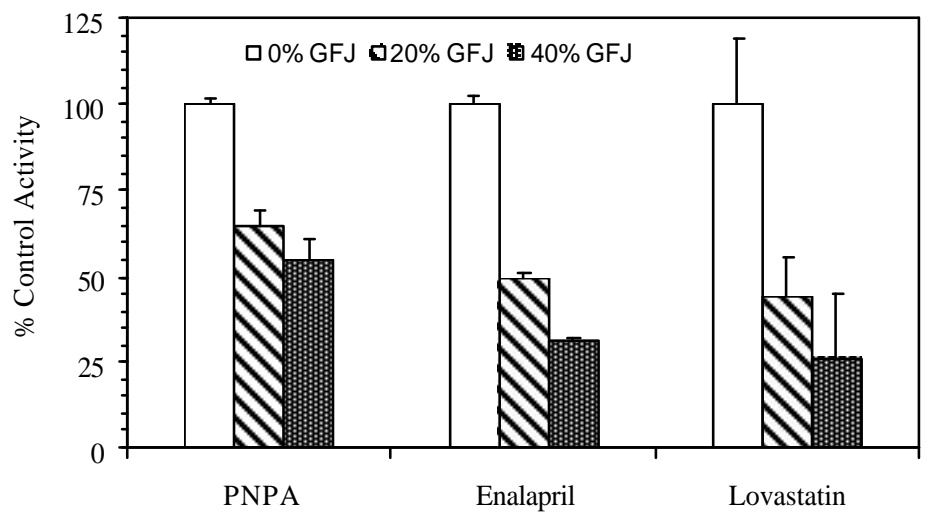

Figure 2. Inhibition of purified porcine esterase activity by GFJ 

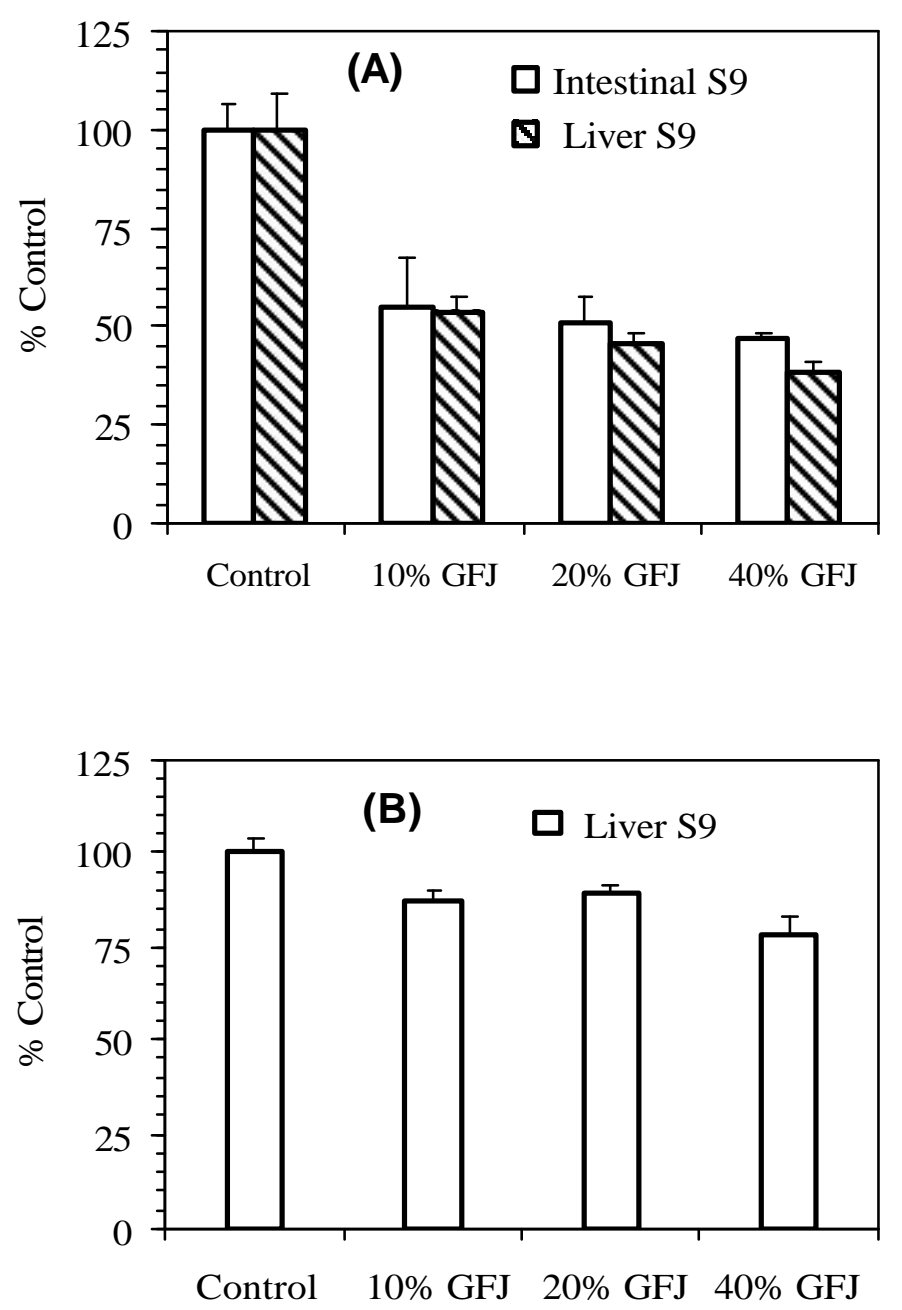

Figure 3. Inhibition of hydrolysis of (A)lovastatin ( $5 \mu M)$ and (B) enalapril $(5 \mu \mathrm{M})$ in human intestinal and liver $\mathrm{S9}$ 

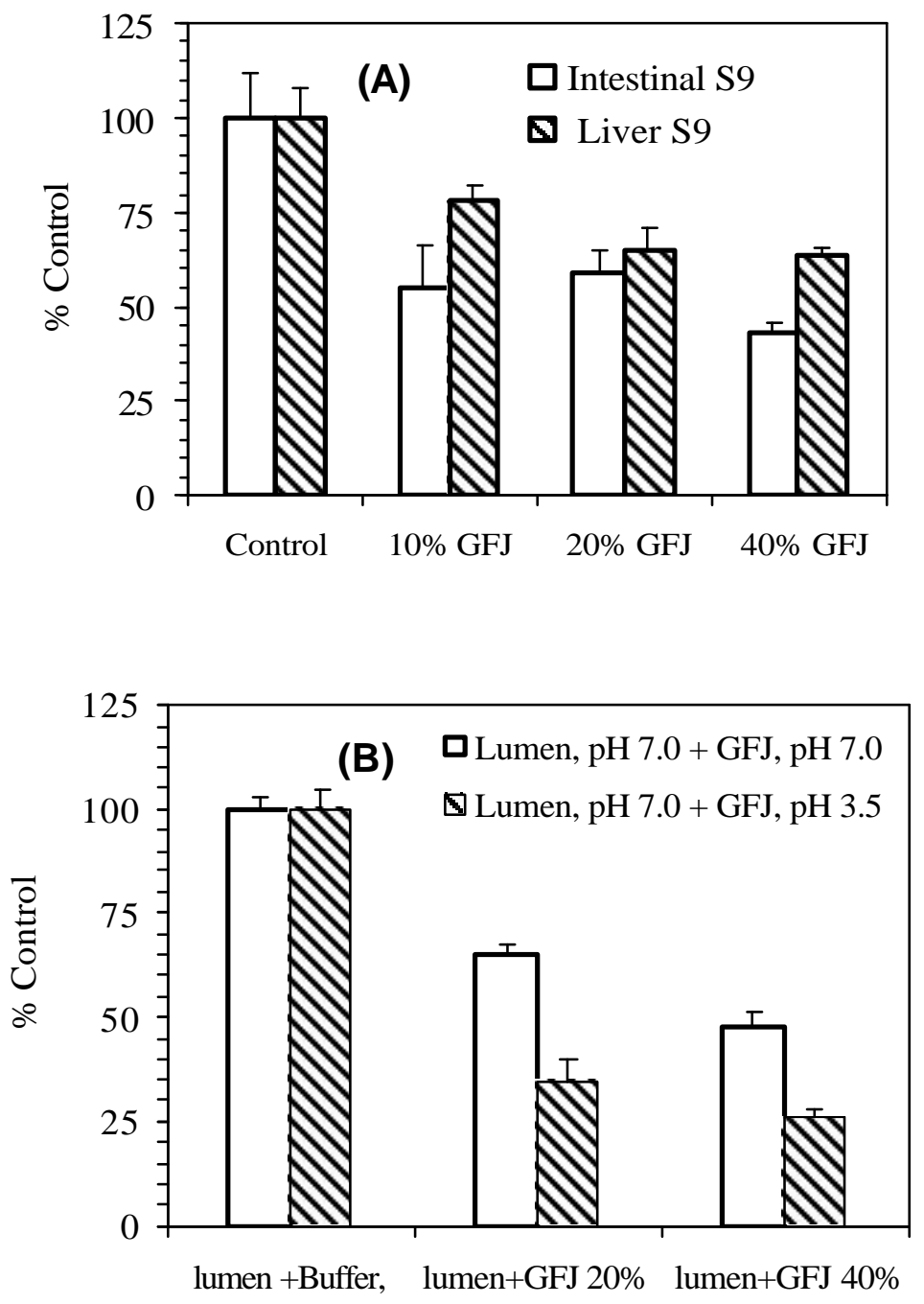

Figure 4. Inhibition of hydrolysis of lovastatin $(5 \mu \mathrm{M})$ in $(A)$ rat intestinal and liver S9 (B) intestinal lumen by GFJ 

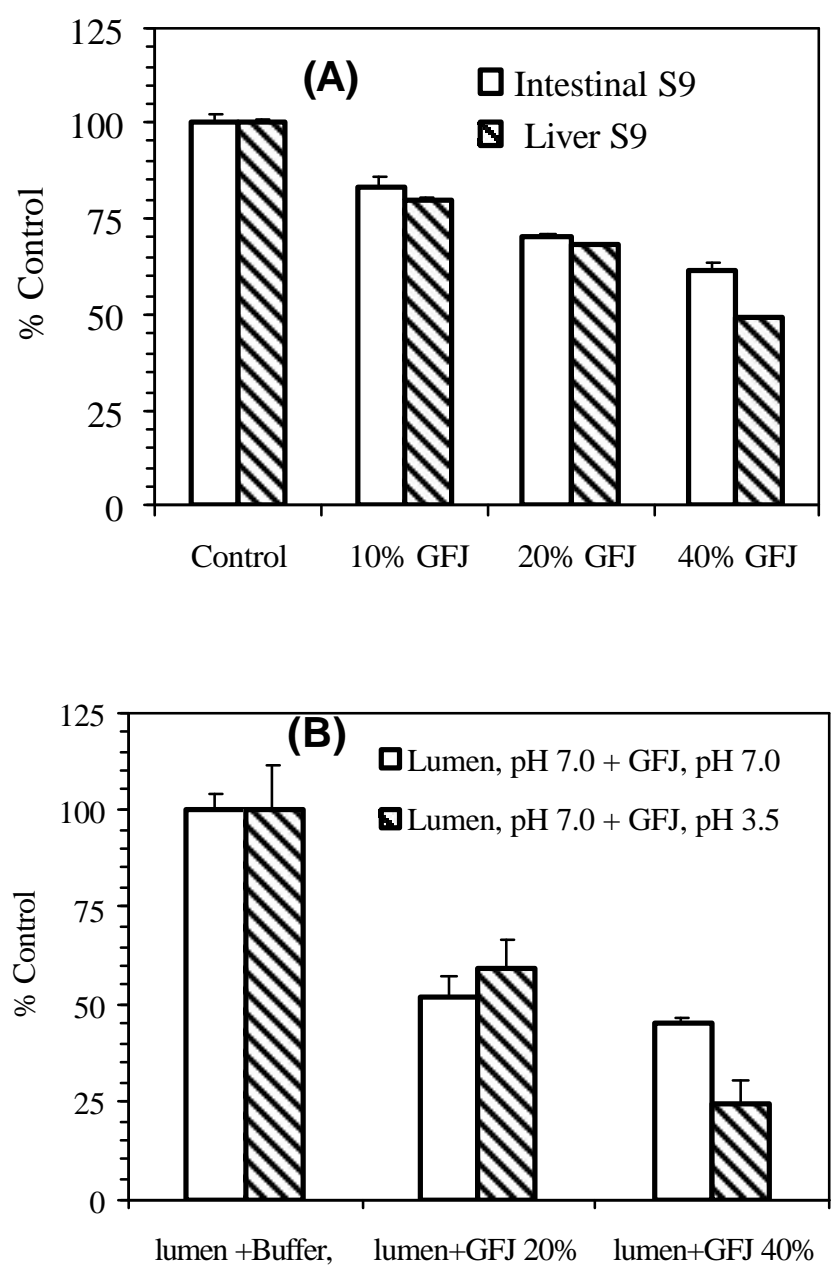

Figure 5. Inhibition of hydrolysis of enalapril $(5 \mu \mathrm{M})$ in $(\mathrm{A})$ rat intestinal and liver S9 (B) rat intestinal lumen by GFJ 




Figure 6. CYP3A activity of Caco-2 homogenates 


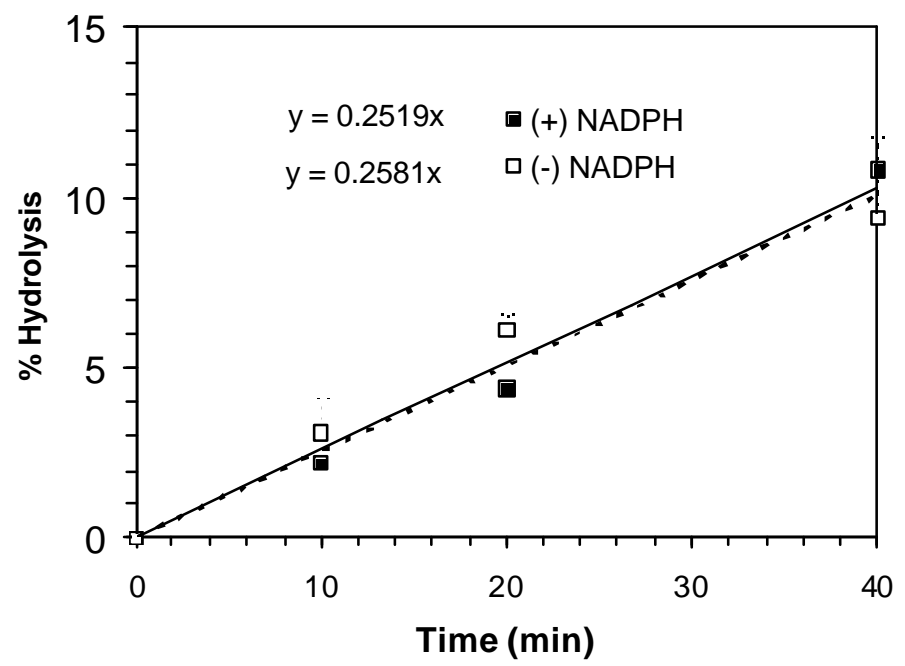

Figure 7. Hydrolysis of lovastatin by Caco-2 homogenates 

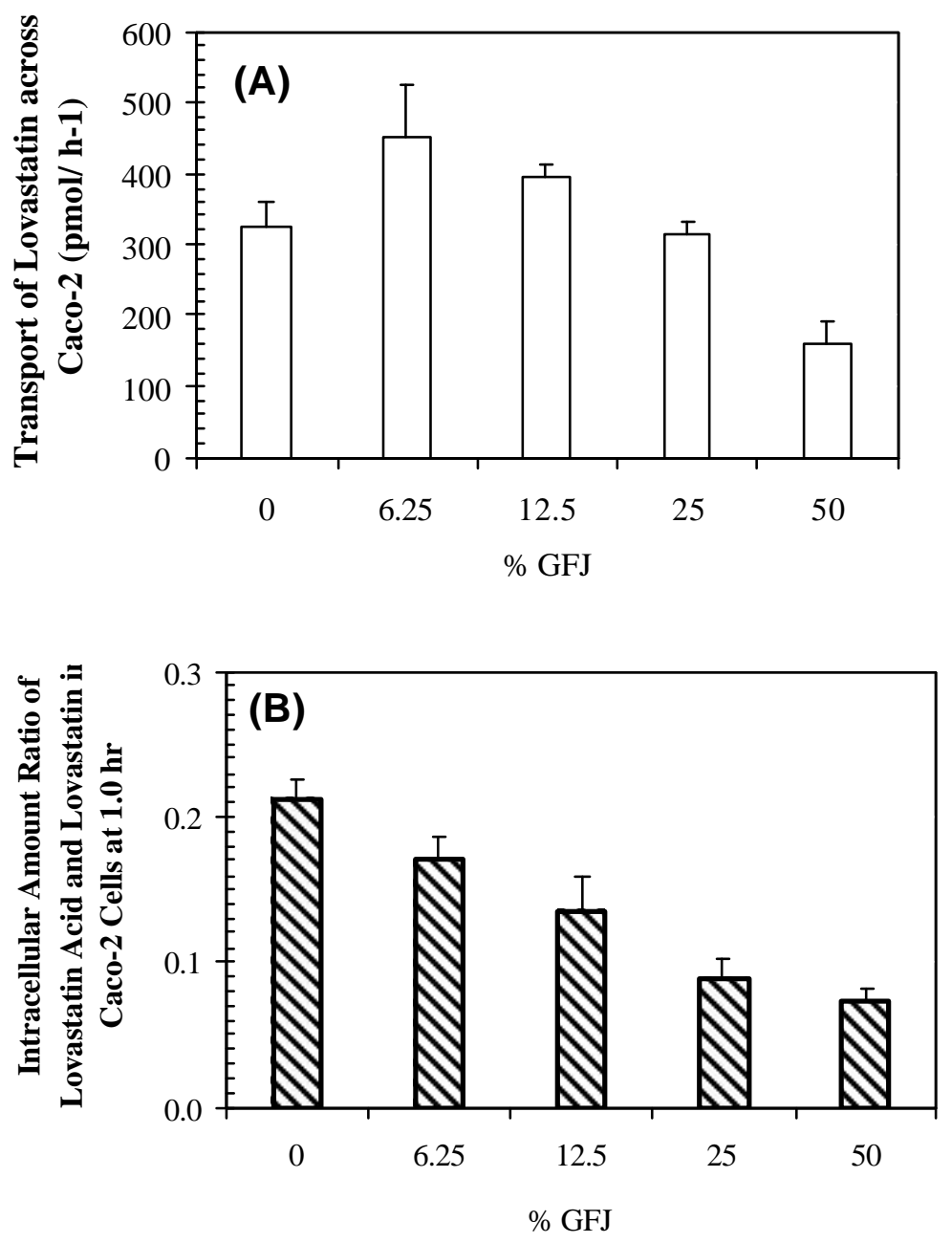

Figure 8. Effect of GFJ on (A) lovastatin $A \rightarrow B$ permeability across Caco-2 membrane (B) ratio of lovastatin acid and lovastatin in Caco-2 cells at $1 \mathrm{hr}$ 



Figure 9. Effect of GFJ on (A) enalapril A $\rightarrow B$ permeability across Caco-2 membrane (B) ratio of enalaprilat and enalapril in Caco-2 cells at $1 \mathrm{hr}$ 



Figure10. The uptake rate of enalapril into (A) Caco-2 cells, and the effect of GFJ (B) human PEPT1-expressed transportocytes 
$\square$ TIC of +Q1: from Sample 11 (Sample002(-) NADPH full) of 20060726_Hmic_HySET1.wiff (Turbo...

Max. $1.6 \mathrm{e} 9 \mathrm{cps}$.

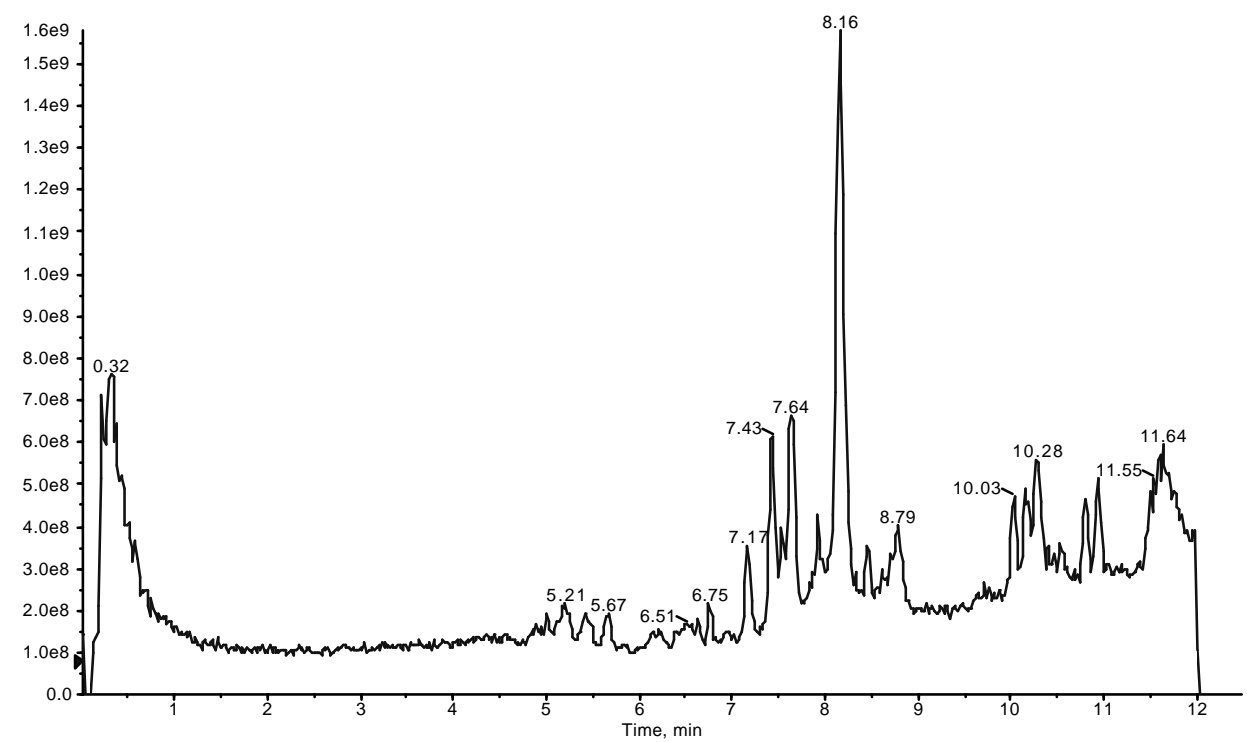

Figure11. Full scan mass chromatogram (150-600 m/z) of incubation mixtures of lovastatin in human liver microsomes w/o NADPH 
$\square$ TIC of + Q1: from Sample 10 (Sample001(NADPH) full) of 20060726_Hmic_HySET1.wiff (Turbo ...



Figure12. Full scan mass chromatogram $(150-600 \mathrm{~m} / \mathrm{z})$ of incubation mixtures of lovastatin in human liver microsomes w/ NADPH 
$\square \mathrm{XIC}$ of $+\mathrm{Q} 1 \mathrm{MI}$ (4 ions): 405.5 amu from Sample 4 (Sample001(NADPH), sim) of 20060726_Hmic..

Max. $1.7 \mathrm{e} 6 \mathrm{cps}$

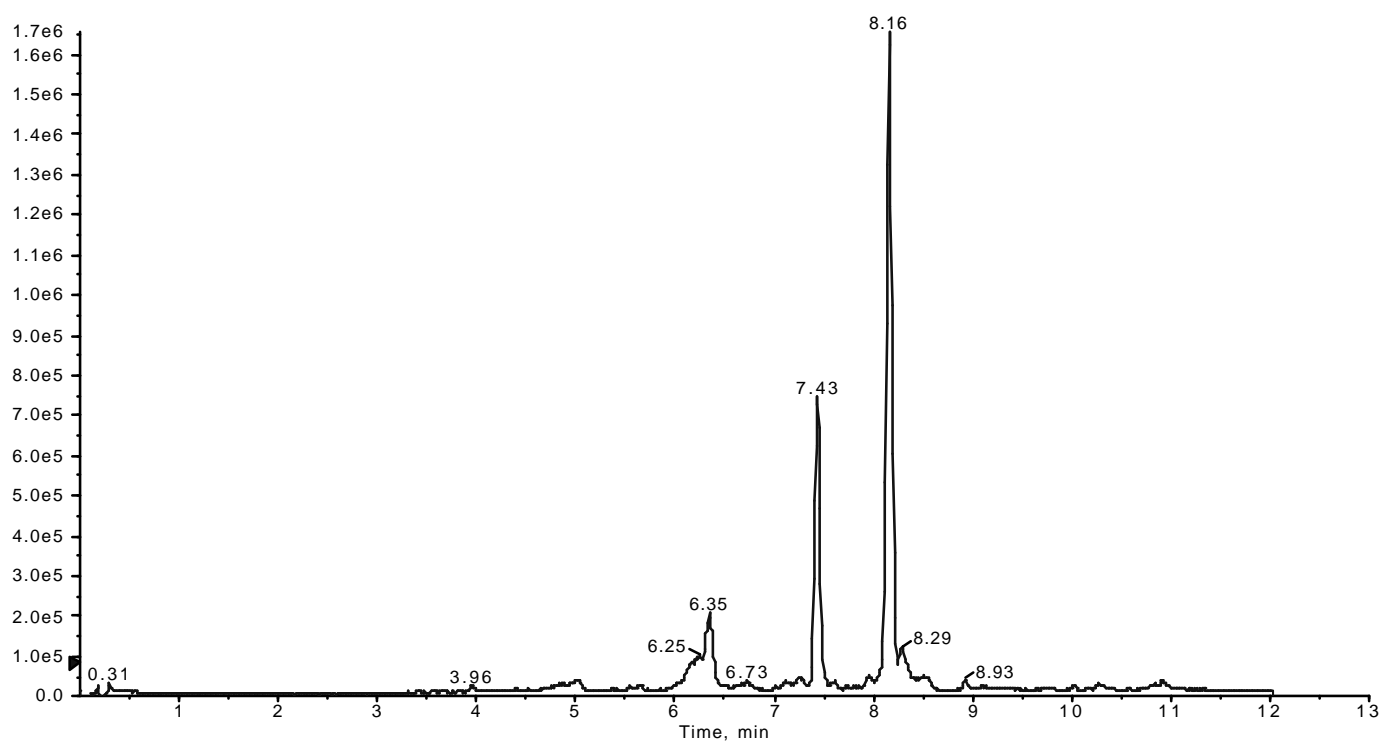

Figure13. SIM scan (m/z 405.5) of incubation mixtures of lovastatin in human liver microsomes $\mathrm{w} / \mathrm{NADPH}$ 
$\square$ TIC of +MS2 (423.40) CE (30): from Sample 14 (Sample001(NADPH) full) of 20060726_Hmic_H...

Max. 3.1e6 cps.

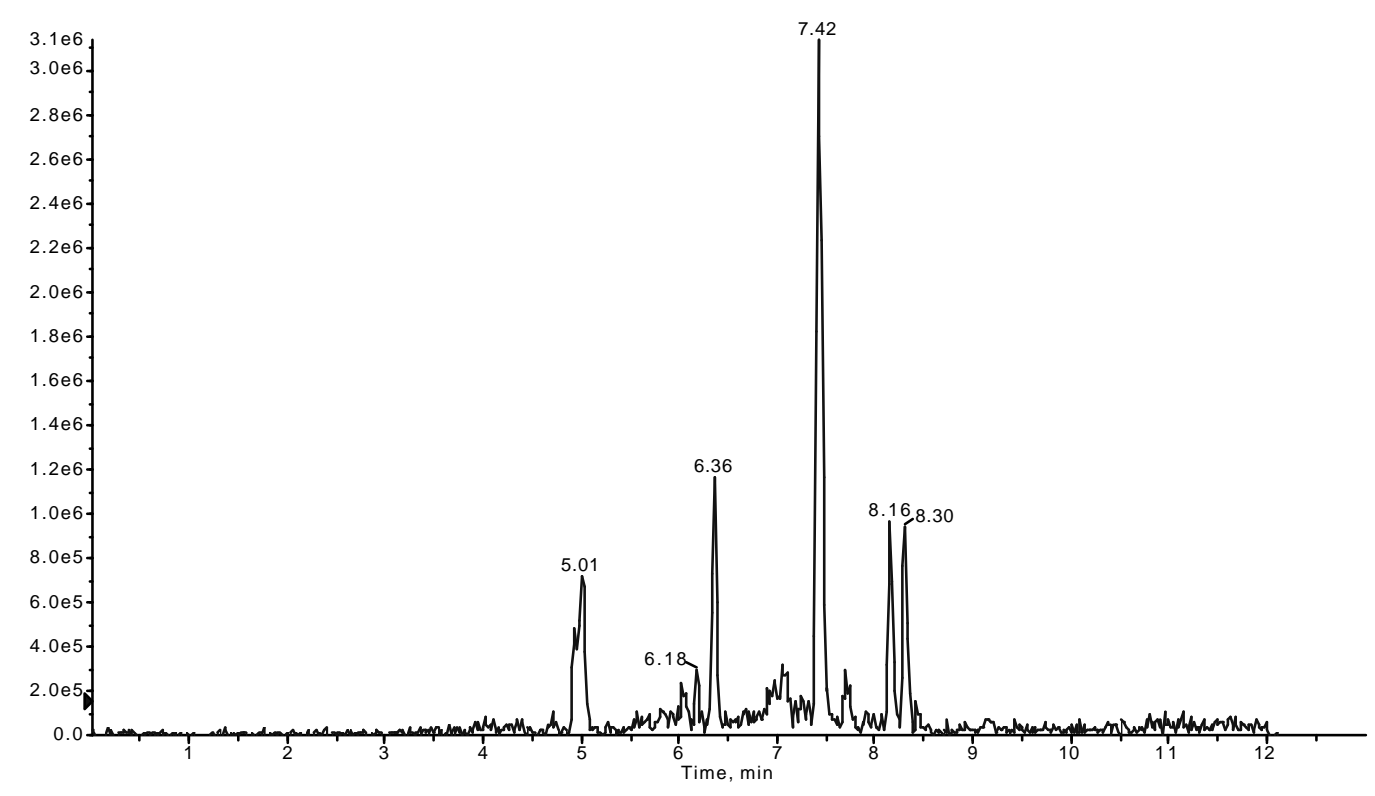

Figure14. SIM scan (m/z 423.4) of incubation mixtures of lovastatin in human liver microsomes $w / N A D P H$ 


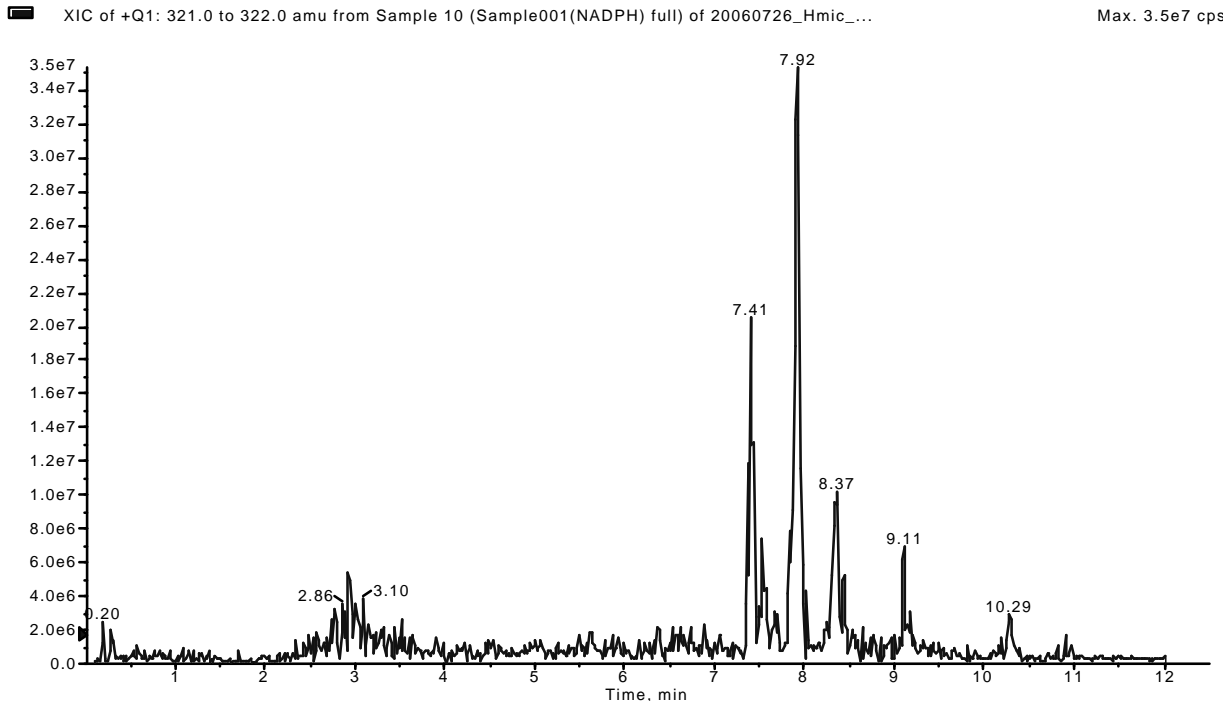

Figure15. Single ion $(\mathrm{m} / \mathrm{z} 321.5)$ extracted from full scan $(150-600 \mathrm{~m} / \mathrm{z})$ 
$\square$ XIC of + Q1 MI (4 ions): 421.3 amu from Sample 4 (Sample001(NADPH), sim) of 20060726_Hmic..

Max. $4.2 \mathrm{e} 6 \mathrm{cps}$.

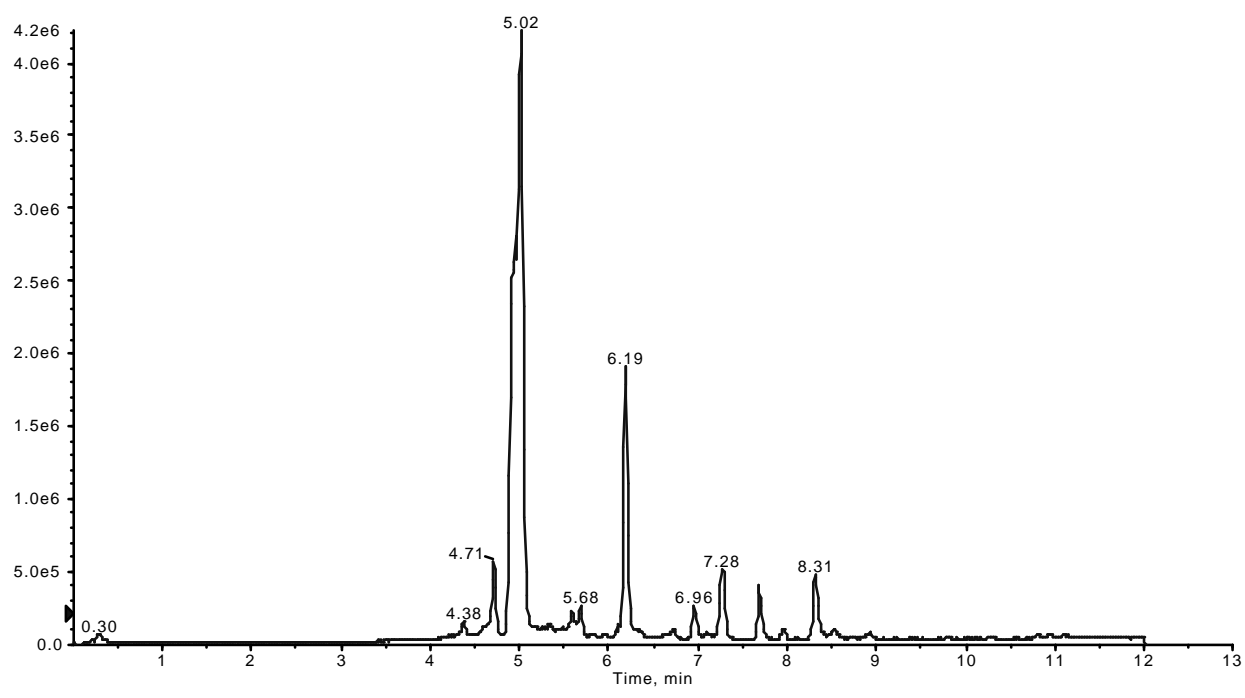

Figure16. SIM scan ( $\mathrm{m} / \mathrm{z}$ 421.4) of incubation mixtures of lovastatin in human liver microsomes $w / N A D P H$ 


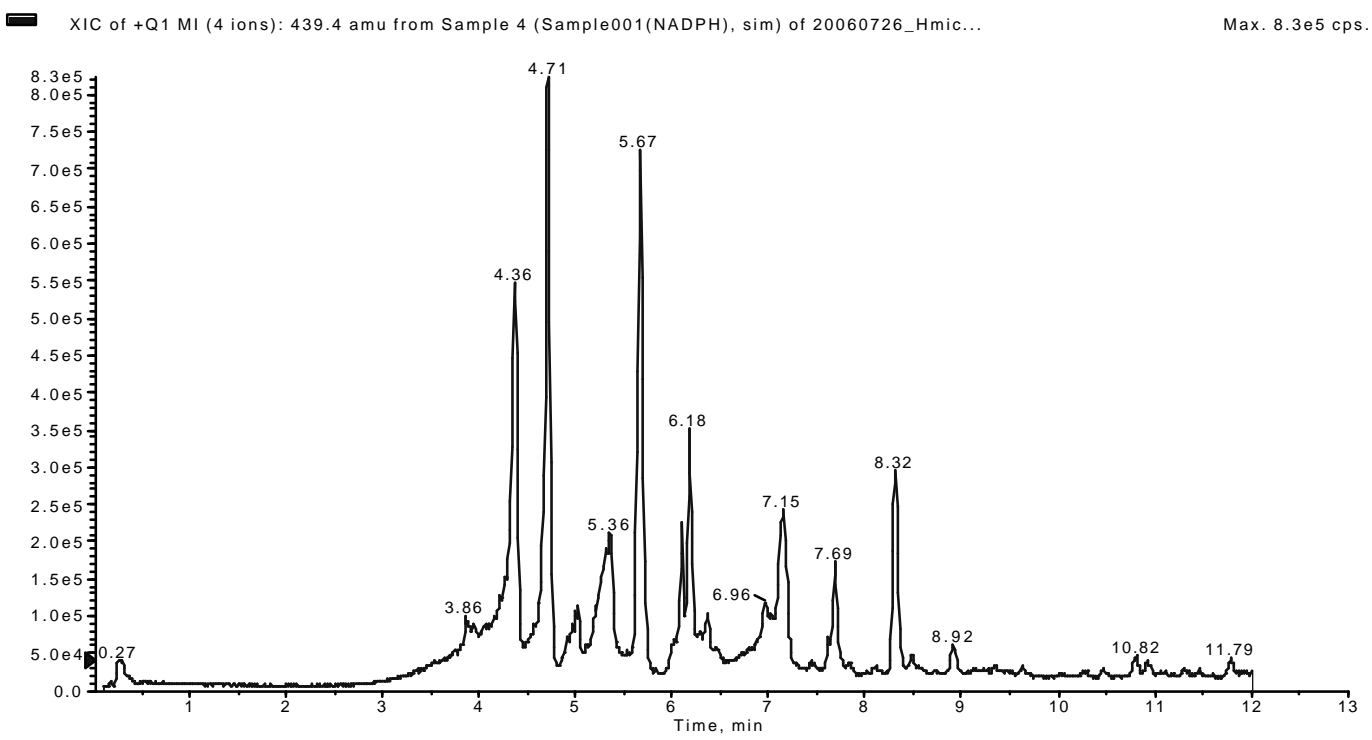

Figure 17. SIM scan ( $\mathrm{m} / \mathrm{z} 439.4)$ of incubation mixtures of lovastatin in human liver microsomes w/ NADPH 

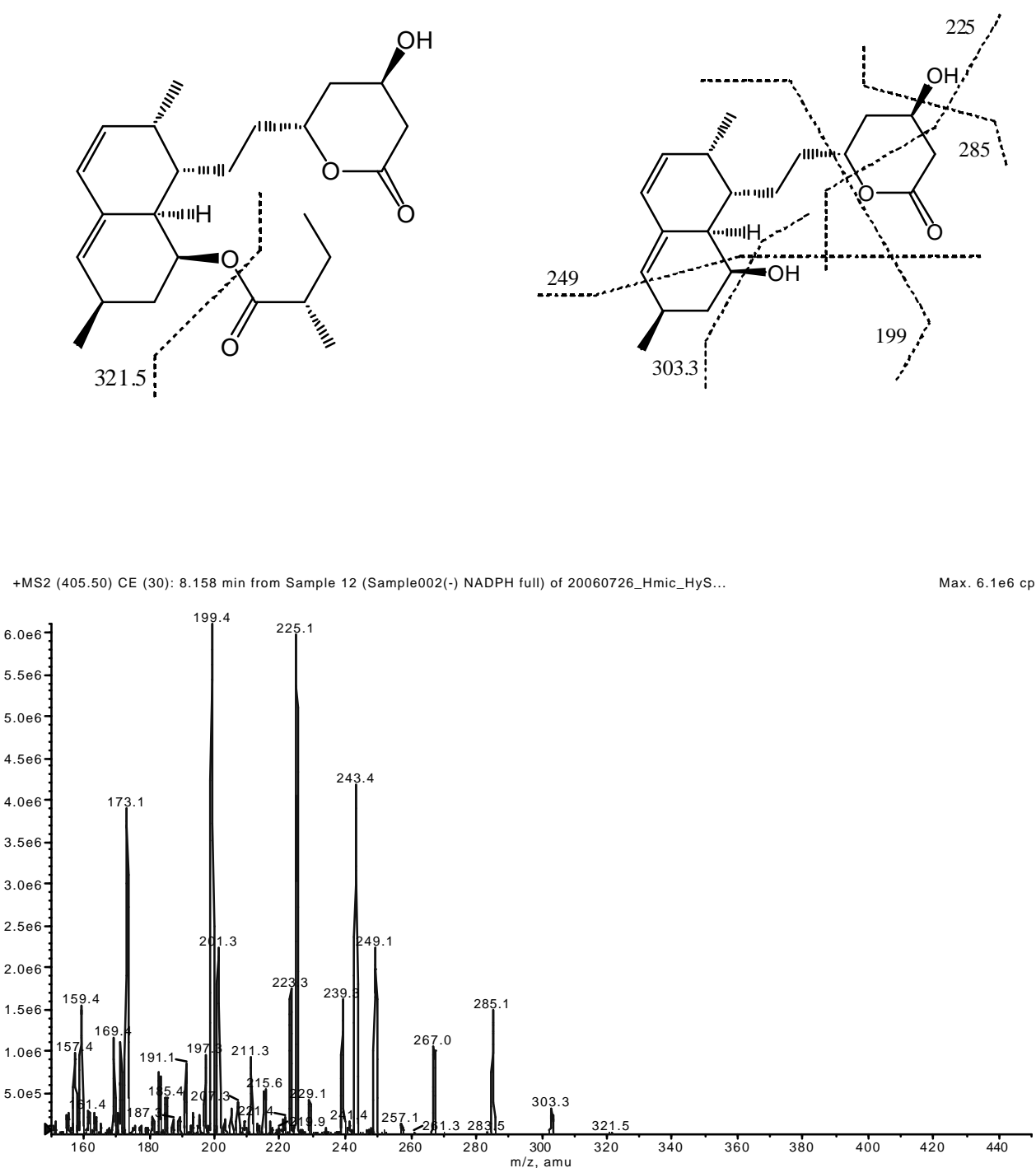

Figure 18. MS/MS of lovastatin ( $\mathrm{m} / \mathrm{z} 405.3$ ) 
口+MS2 (423.40) CE (30): 7.354 to $7.521 \mathrm{~min}$ from Sample 14 (Sample001(NADPH) full) of 20060726_Hm...

Max. $2.5 \mathrm{e} 4 \mathrm{cps}$

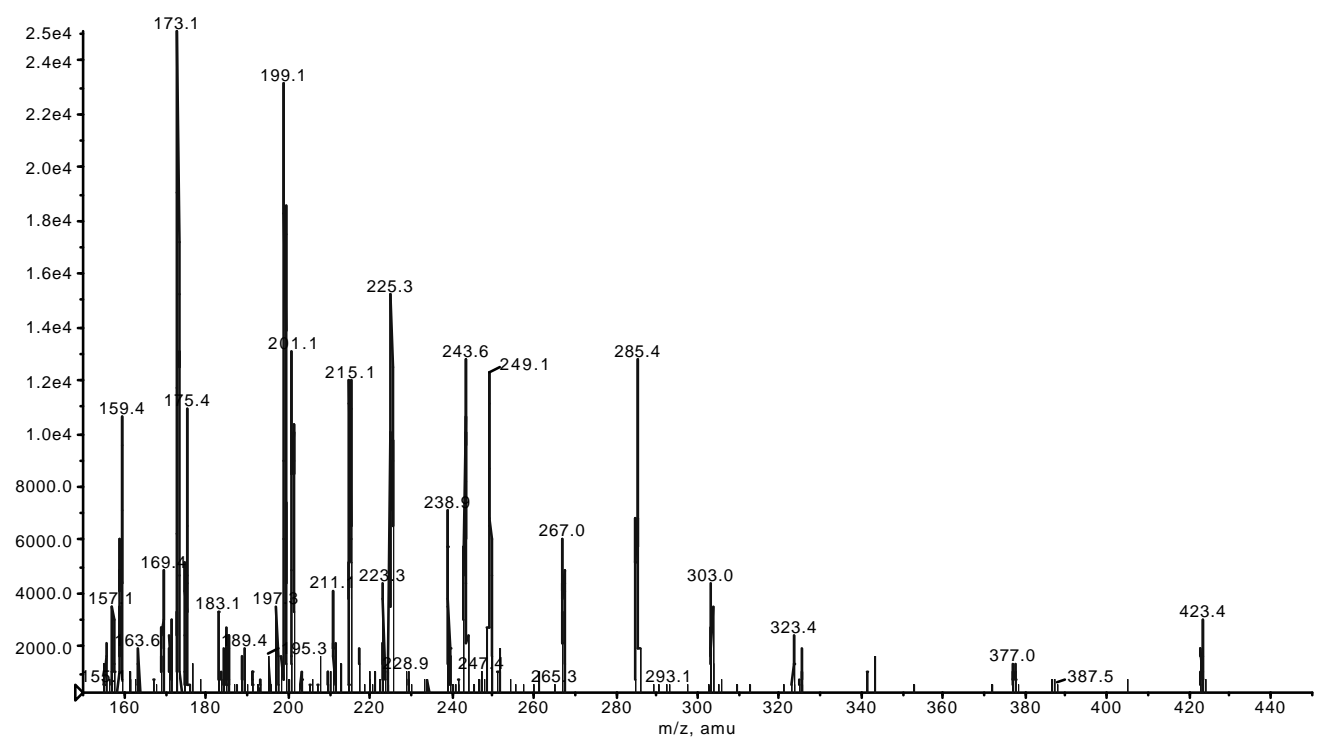

Figure19. MS/MS of lovastatin acid ( $\mathrm{m} / \mathrm{z}$ 423.3) 

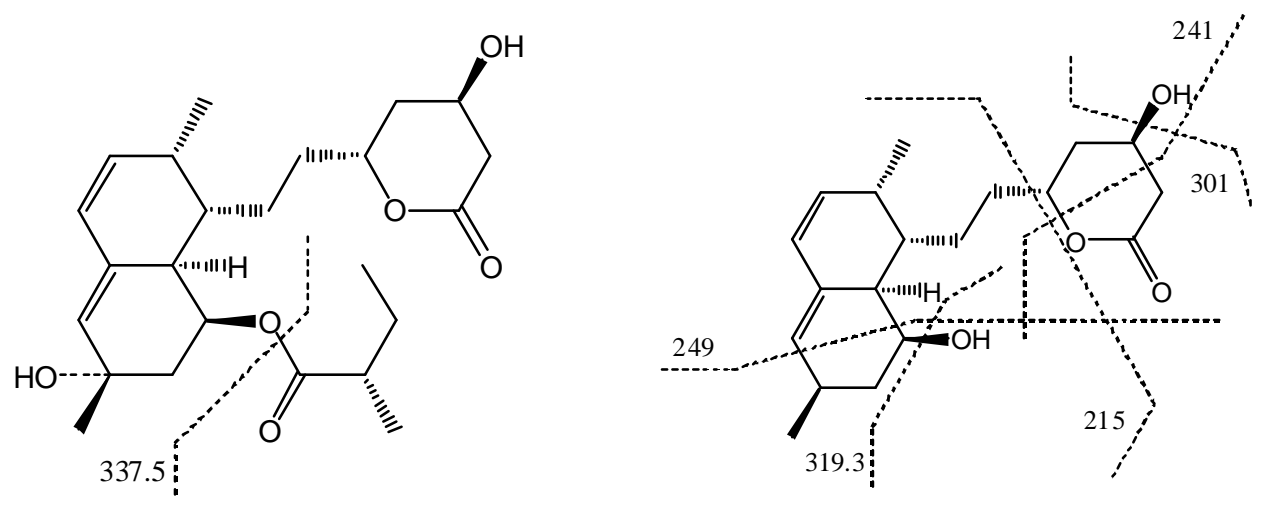

口

+MS2 (421.30) CE (30): 4.975 to $5.109 \mathrm{~min}$ from Sample 13 (Sample001(NADPH) full) of 20060726_Hm...

Max. $1.5 \mathrm{e} 5 \mathrm{cps}$

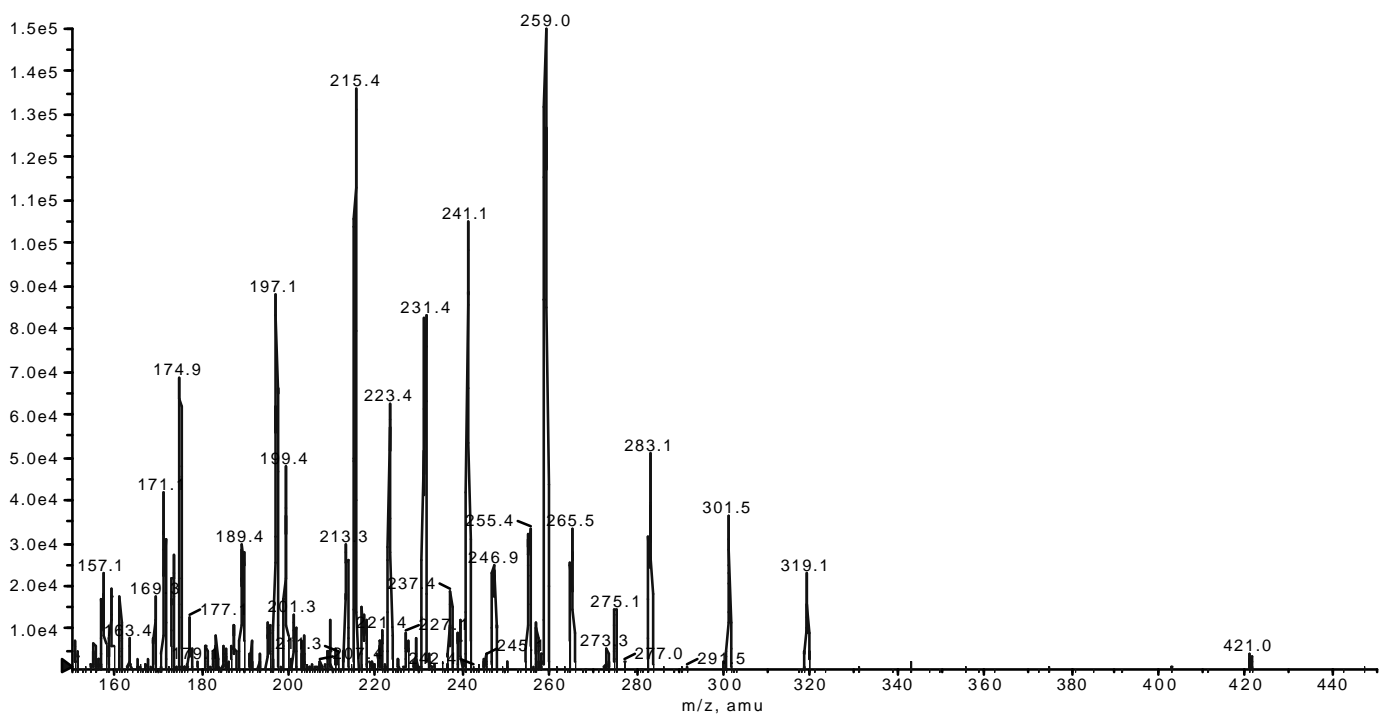

Figure 20. MS/MS of hydroxylovastatin $\mathrm{m} / \mathrm{z} 421.3$, retention time $\left(\mathrm{t}_{\mathrm{R}}\right) 5.02 \mathrm{~min}$ 
ש +MS2 (421.30) CE (30): 6.114 to $6.332 \mathrm{~min}$ from Sample 13 (Sample001(NADPH) full) of 20060726_Hm...

Max. $3.9 \mathrm{e} 4 \mathrm{cps}$

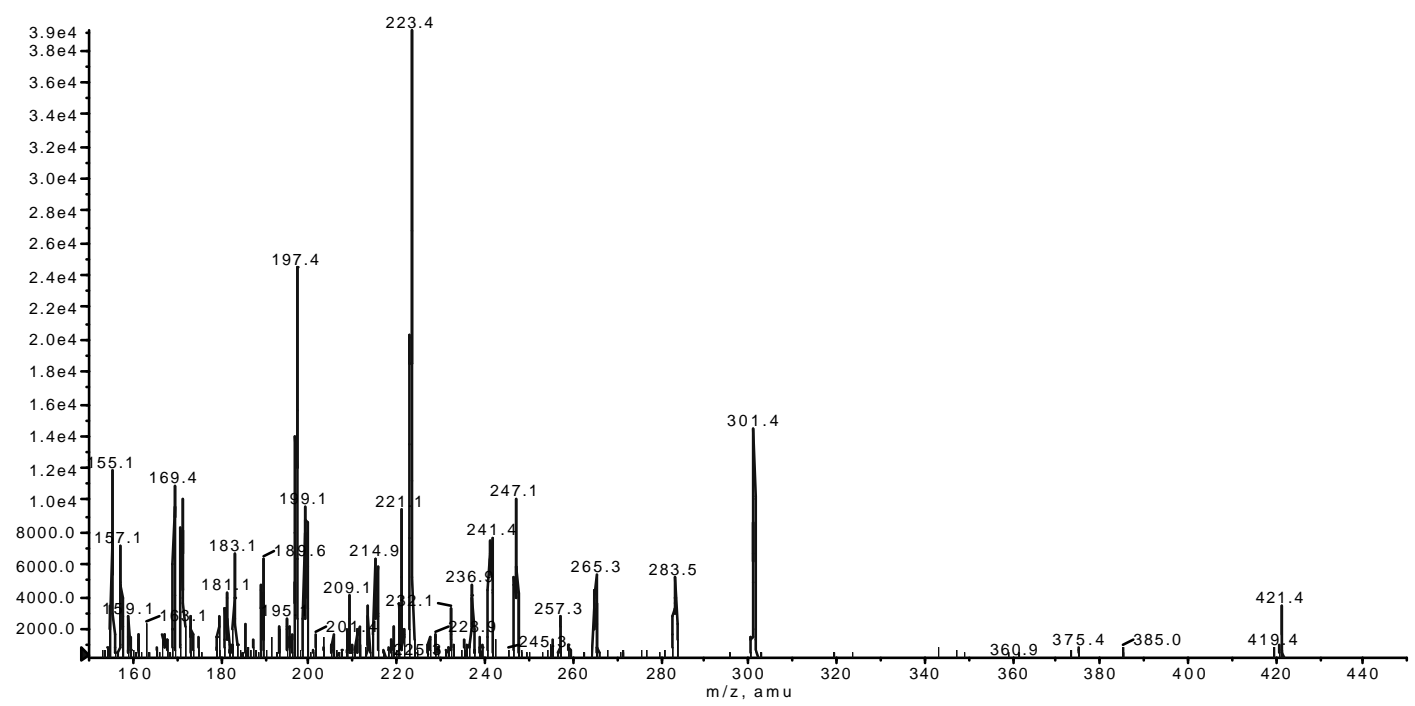

Figure 21. MS/MS of hydroxylovastatin $\mathrm{m} / \mathrm{z} 421.3, \mathrm{t}_{\mathrm{R}} 6.19 \mathrm{~min}$. 
$\square+$ MS2 (439.40) CE (30): 5.528 to $5.829 \mathrm{~min}$ from Sample 15 (Sample001(NADPH) full) of 2006...

Max. $7265.6 \mathrm{cps}$

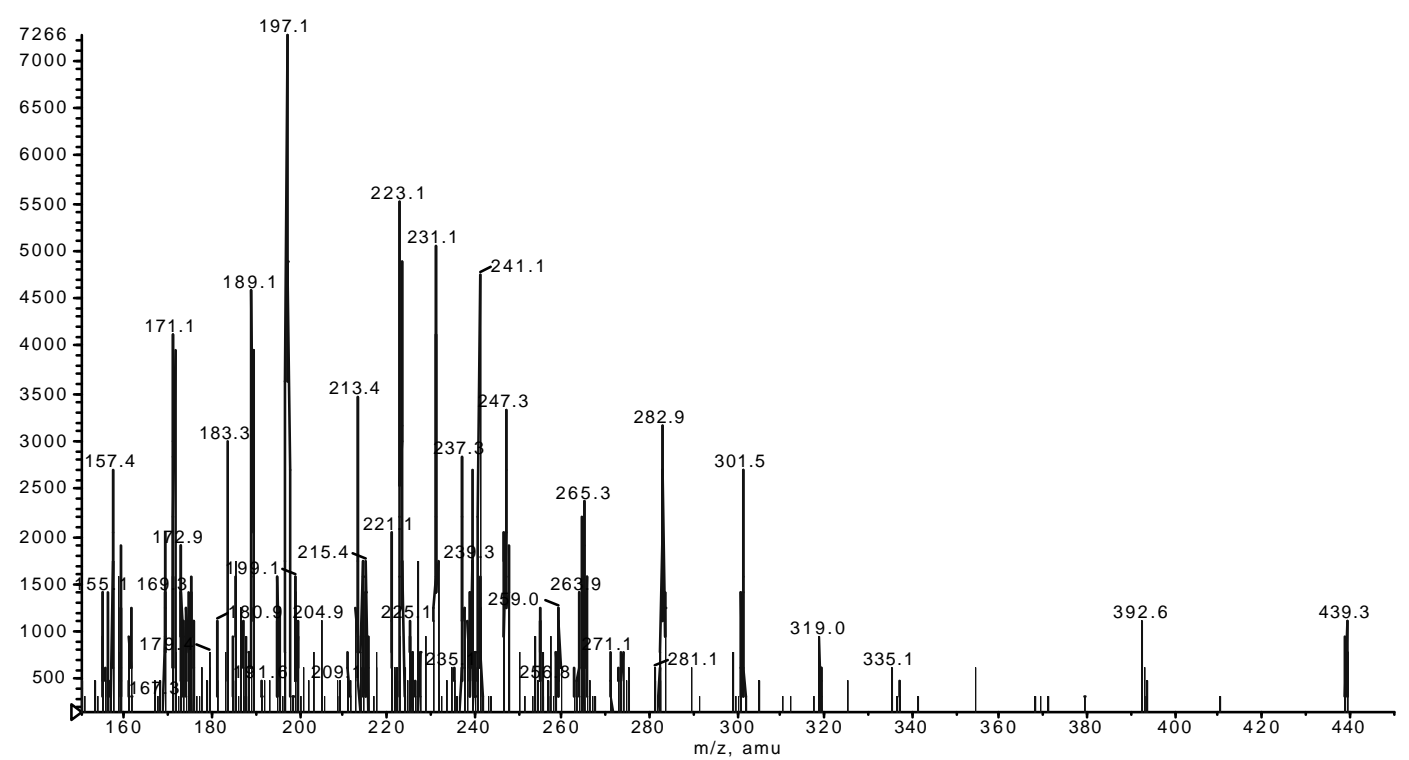

Figure 22. MS/MS of hydroxylovastatin acid m/z 439.3, $t_{R} 4.36$ min. 
口+MS2 (439.40) CE (30): 4.690 to $4.791 \mathrm{~min}$ from Sample 15 (Sample001(NADPH) full) of 20060726_Hm...

Max. $2.1 \mathrm{e} 4 \mathrm{cps}$

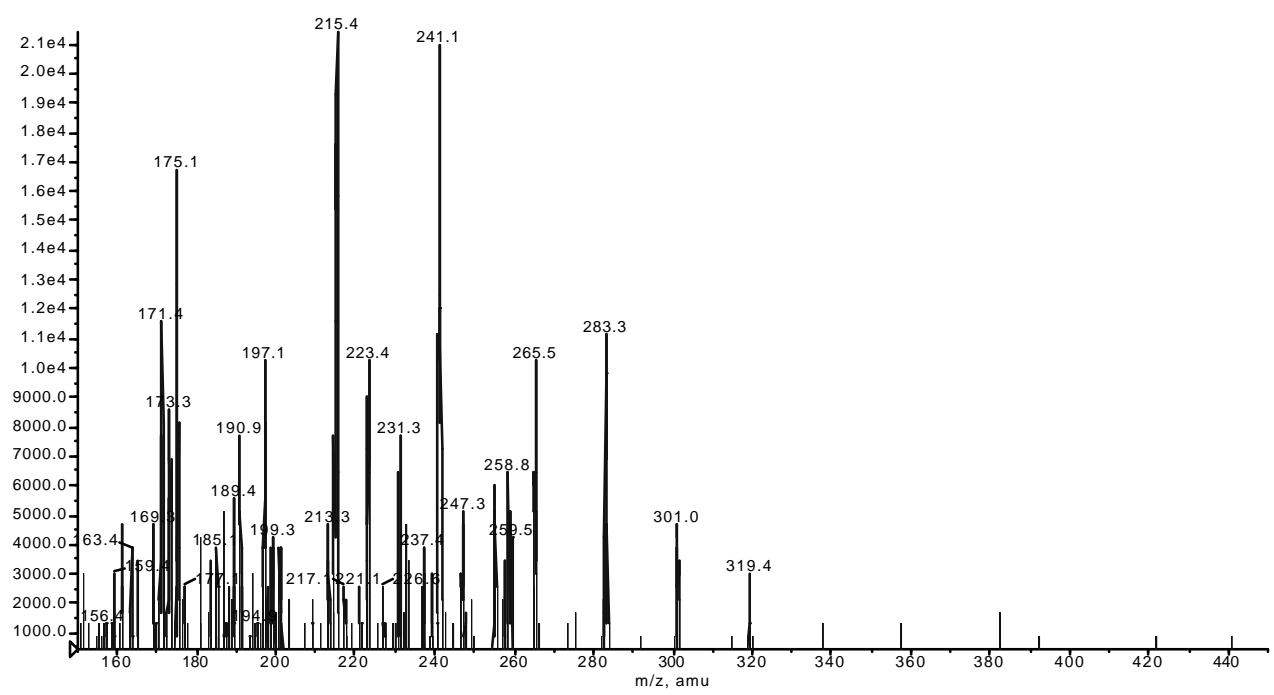

Figure 23. MS/MS of hydroxylovastatin acid $\mathrm{m} / \mathrm{z} 439.3, \mathrm{t}_{\mathrm{R}} 5.67 \mathrm{~min}$. 



Figure 24. Effect of the esterase inhibitor BNPP (25 mg/kg PO) on the plasma concentration-time profiles of $(A)$ enalaprilat and $(B)$ lovastatin acid following oral administration of enalapril and lovatatin at $10 \mathrm{mg} / \mathrm{kg}$, respectively 



Figure 25. Plasma concentration-time profiles of $(A)$ enalaprilat and (B)lovastatin acid following oral administration of enalapril and lovastatin at $10 \mathrm{mg} / \mathrm{kg}$ with water or GFJ (1:3. 1:2, Concentrate), respectively 



Figure 26. Portal plasma concentration-time profiles of (A) lovastatin acid (B) lovastatin acid + lovastatin following oral administration of lovastatin $(10 \mathrm{mg} / \mathrm{kg})$ with water $(\mathrm{pH} 3.5)$ or GFJ to portal vein-cannulated rats, pretreated at -15 and $-2 \mathrm{hr}$ with water $(\mathrm{pH} 3.5)$ or GFJ 



Figure 27. Portal plasma concentration-time profiles of (A) 6' $\$$-hydroxy lovastatin (B) 6'乃 -hydroxylovastatin acid following oral administration of lovastatin $(10 \mathrm{mg} / \mathrm{kg}$ ) with water ( $\mathrm{pH} 3.5)$ or GFJ to portal vein-cannulated rats, pretreated at -15 and $-2 \mathrm{hr}$ with water $(\mathrm{pH} 3.5)$ or GFJ

Both 6'ß-hydroxylovastatin and 6'ß-hydroxylovastatin acid was estimated using lovastatin as standard 


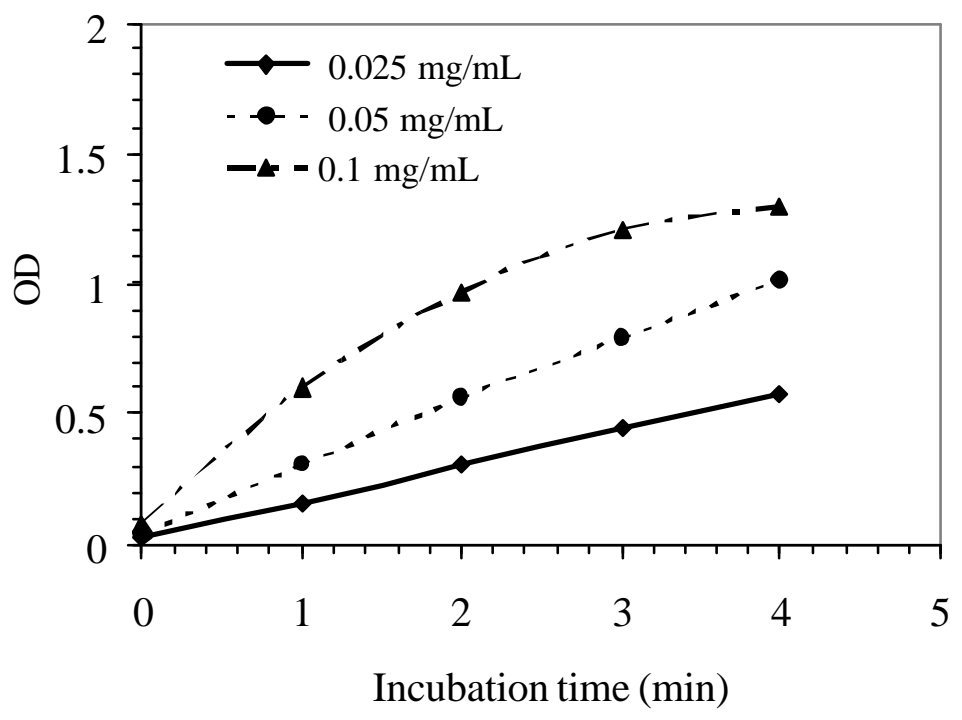

Figure 28. PNPA hydrolysis by human liver microsomes 

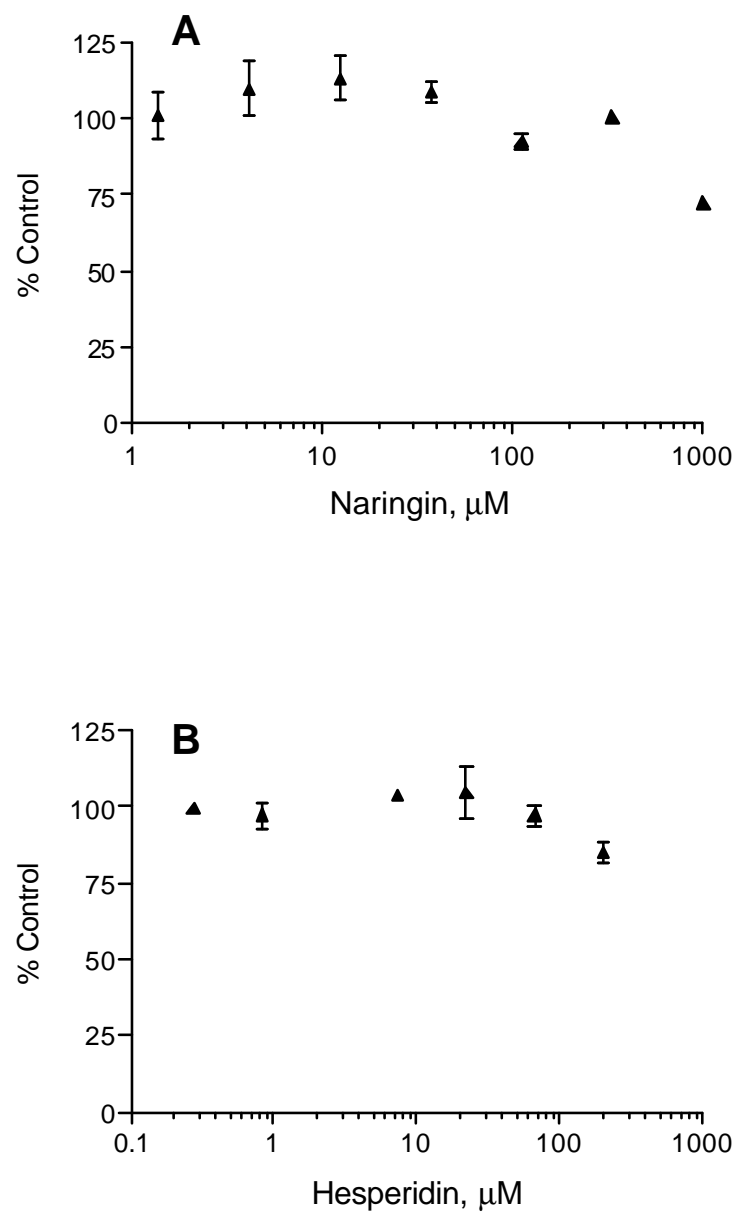

Figure 29. Inhibition potential of purified porcine esterases by $(A)$ naringin and $(\mathrm{B})$ hesperidin 



Figure.30 Inhibition potential of purified porcine esterases by $(A)$ bergamottin (B) 6',7'-dihydroxybergamottin, and (C) bergapten 

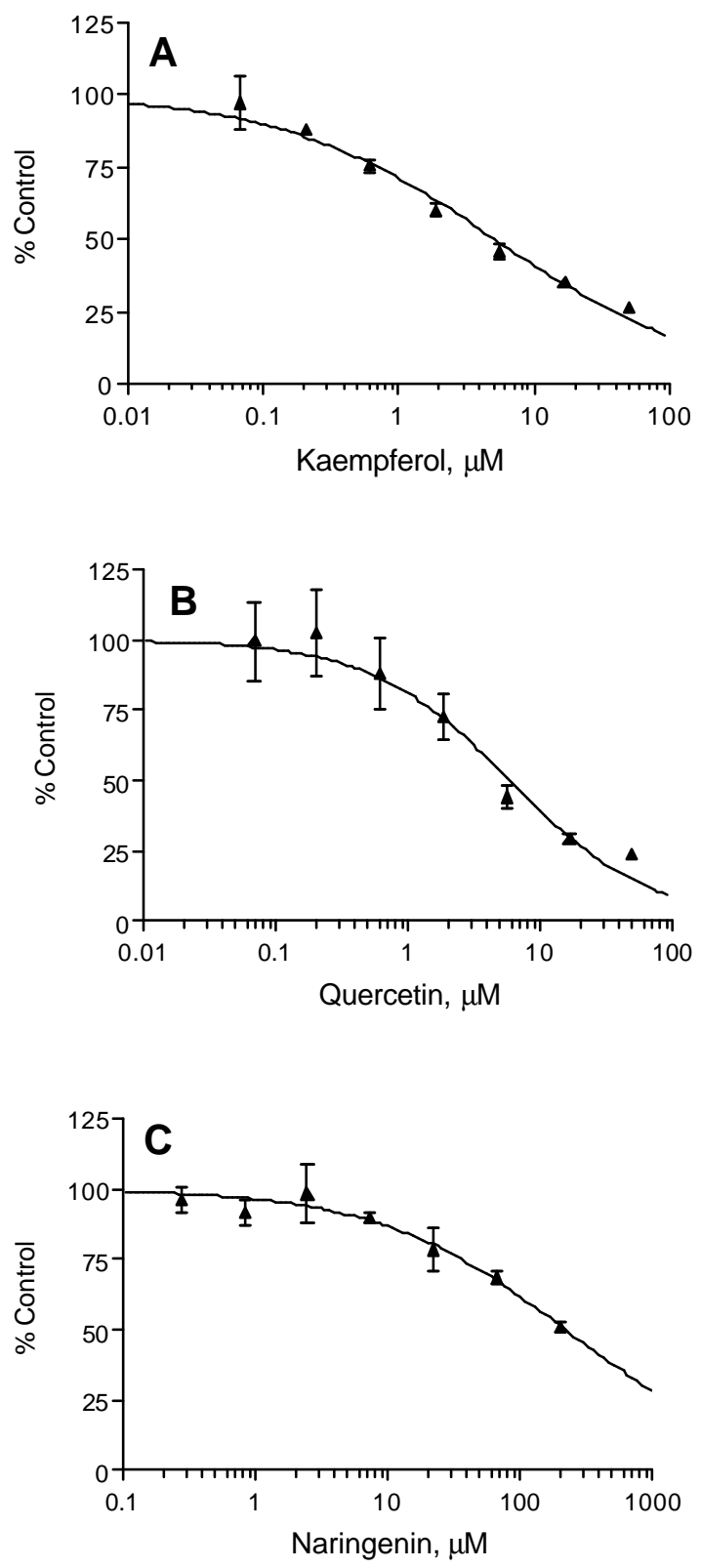

Figure 31. Inhibition potential of purified porcine esterases by (A) kaempferol (B) quercetin, and (C) naringenin 

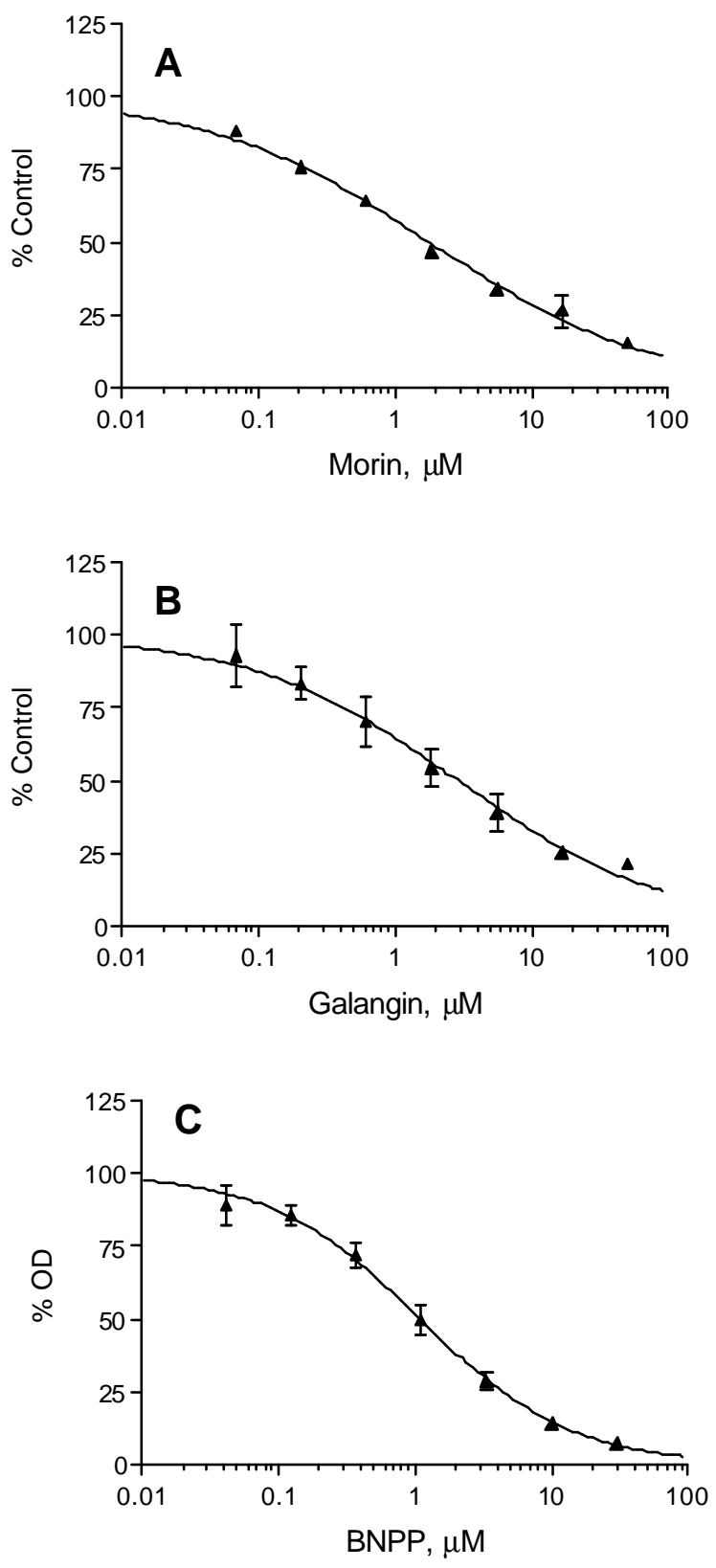

Figure 32. Inhibition potential of purified porcine esterases by $(A)$ morin (B) galangin, and (C) BNPP 

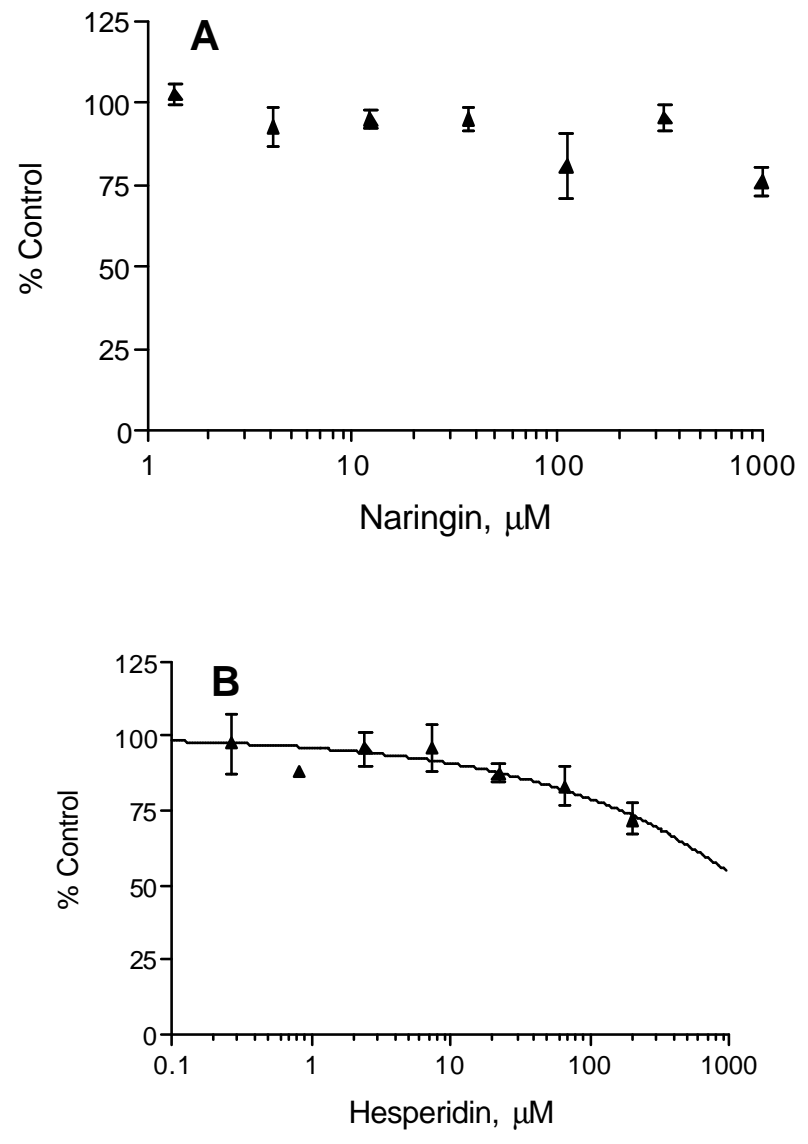

Figure 33. Inhibition potential of esterases of human liver microsomes by $(\mathrm{A})$ naringin and $(\mathrm{B})$ hesperidin 

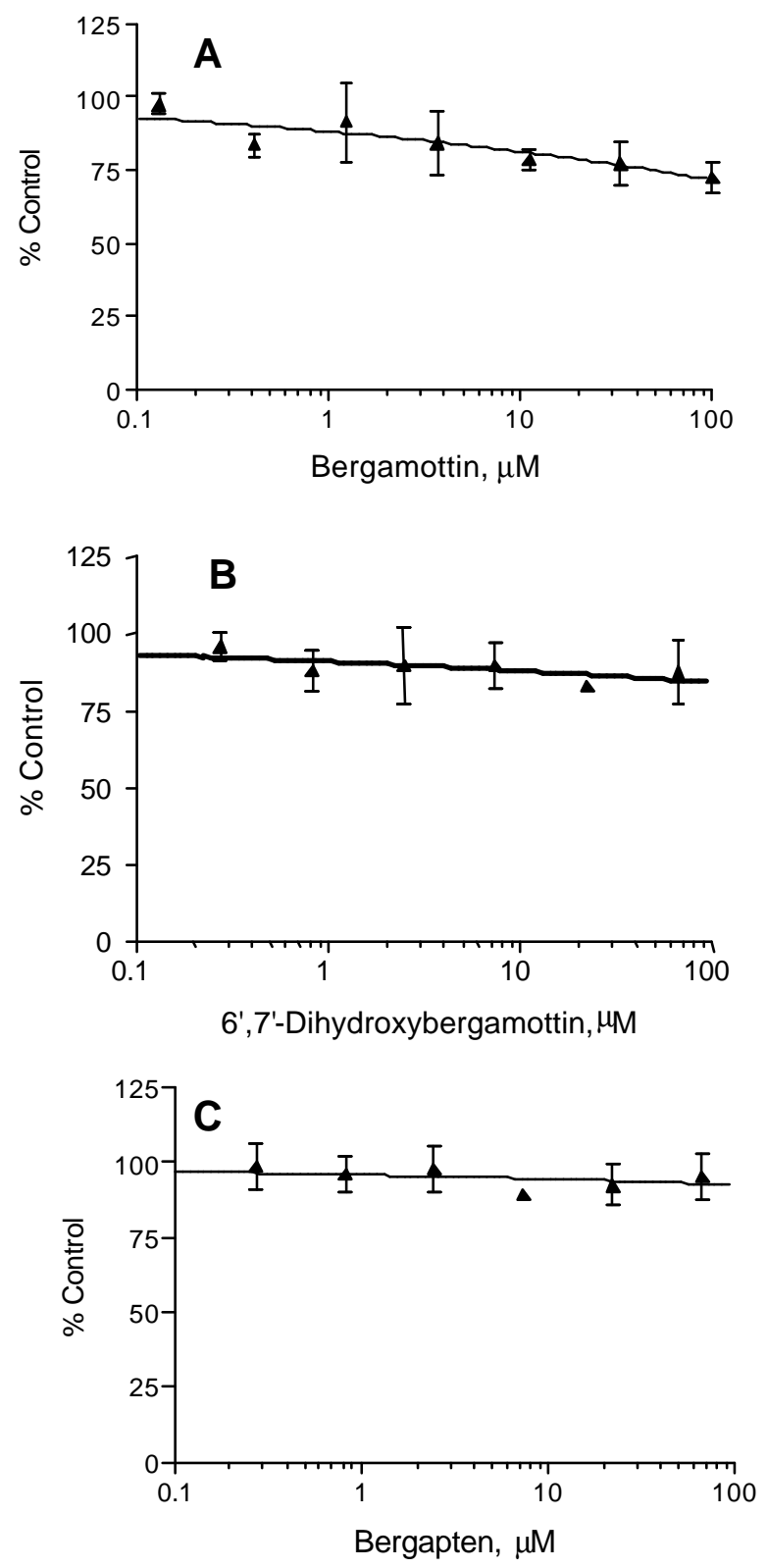

Figure 34. Inhibition potential of esterases of human liver microsomes by (A) bergamottin,(B) 6',7'-dihydroxybergamottin, and (C) bergapten 

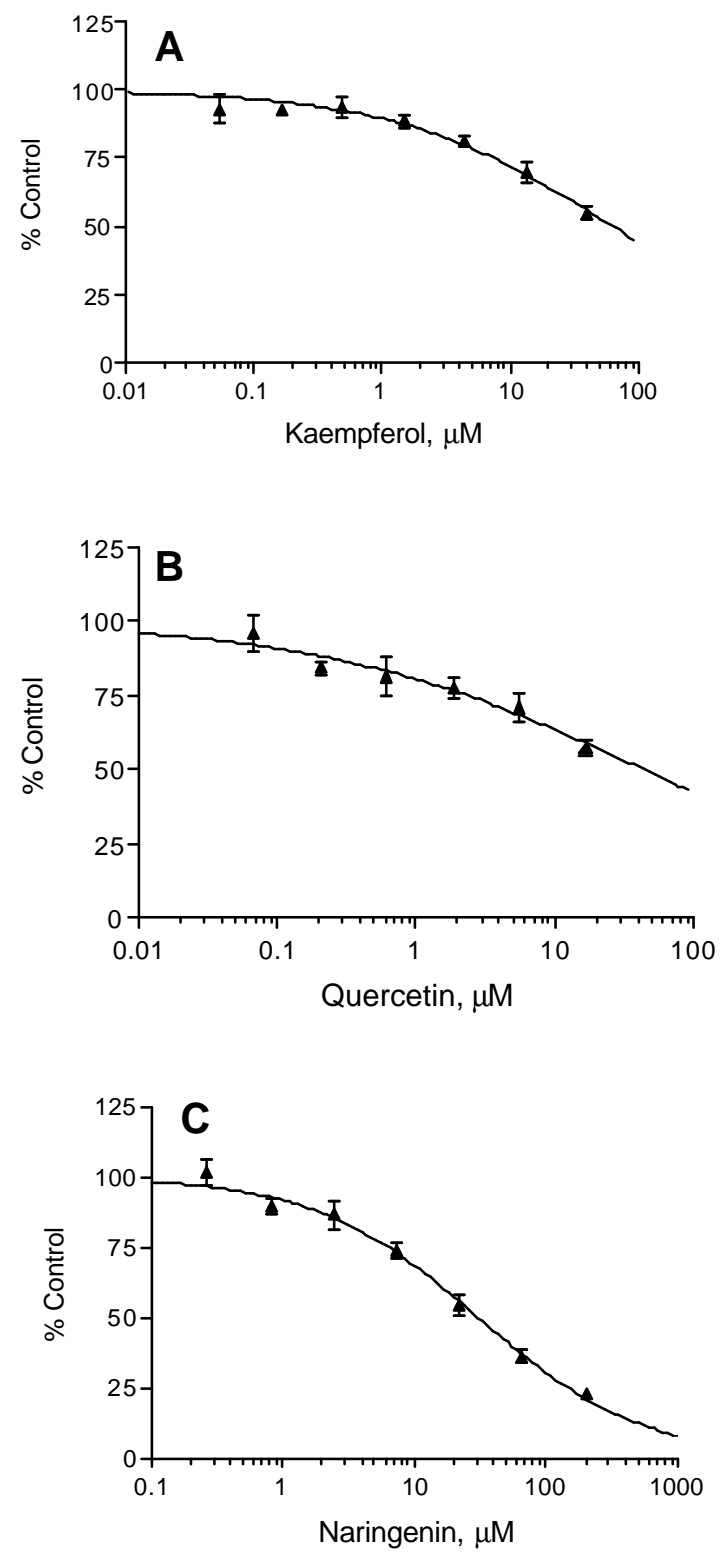

Figure 35. Inhibition potential of esterases of human liver microsomes by $(A)$ kaempferol, $(B)$ quercetin, and $(C)$ naringenin 

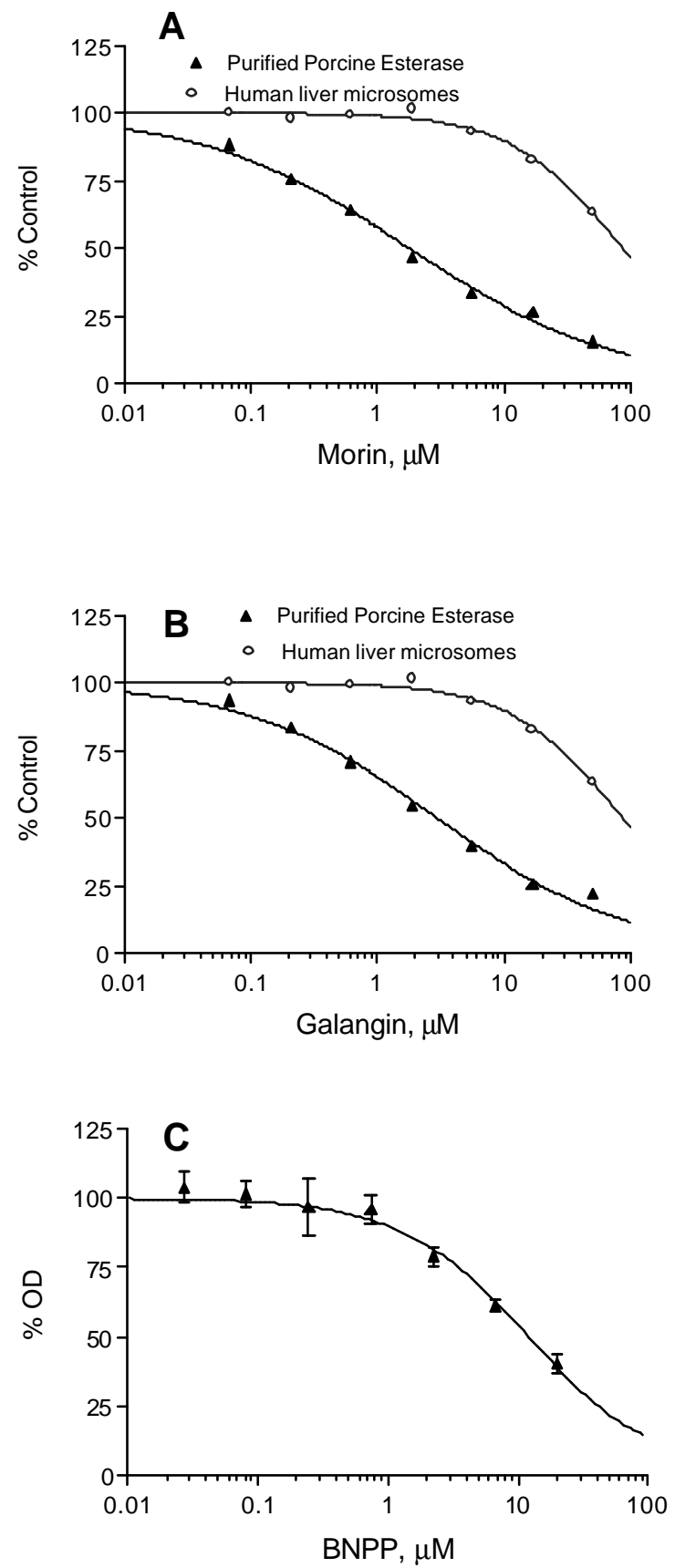

Figure 36. Inhibition of esterases of human liver microsomes by (A) morin, (B) galangin, and (C) BNPP 

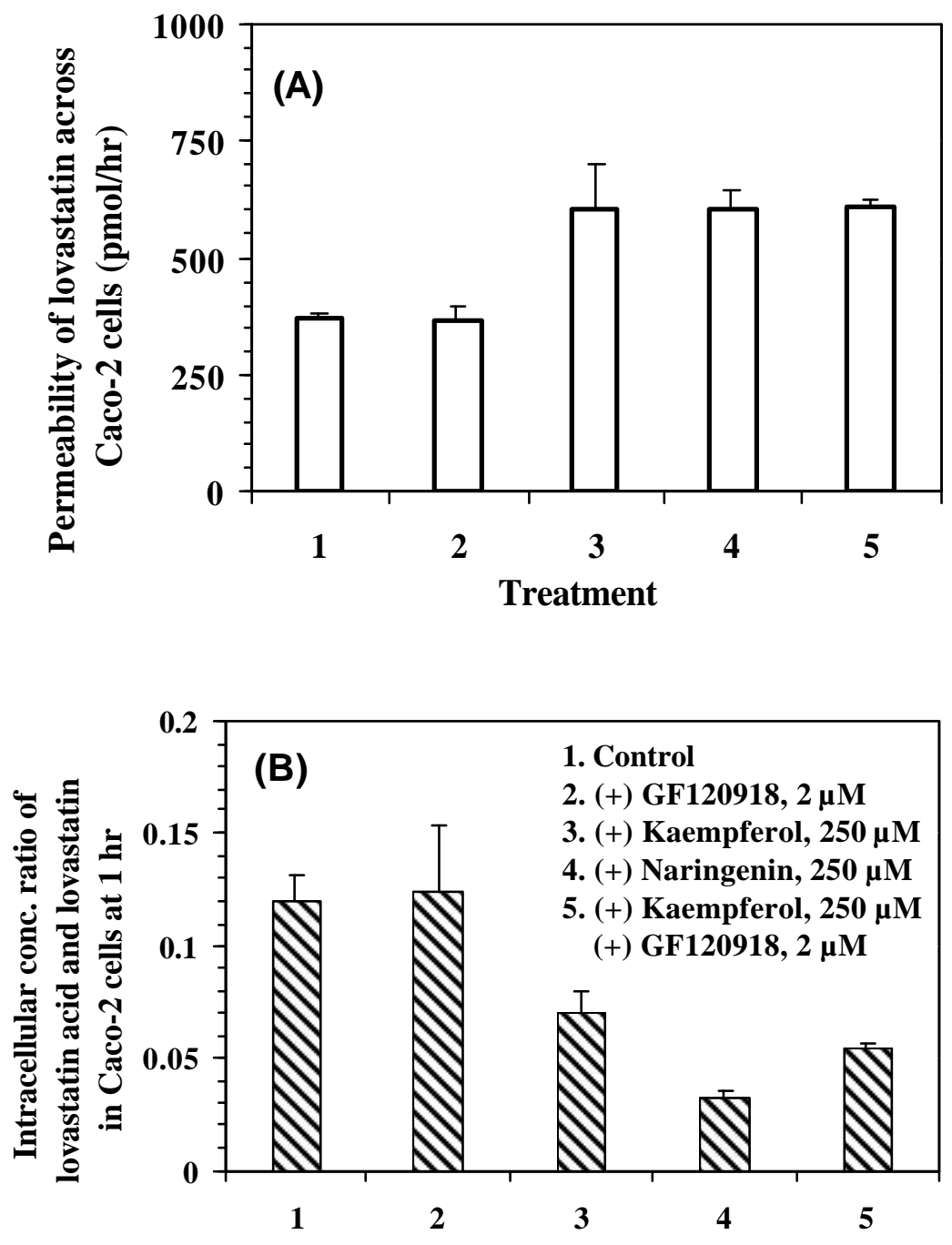

Figure 37. Effect of grapefruit juice constituents on $(A)$ lovastatin $A \rightarrow B$ permeability across Caco-2 membrane (B) ratio of lovastatin acid and lovastatin in Caco-2 cells at $1 \mathrm{hr}$ 

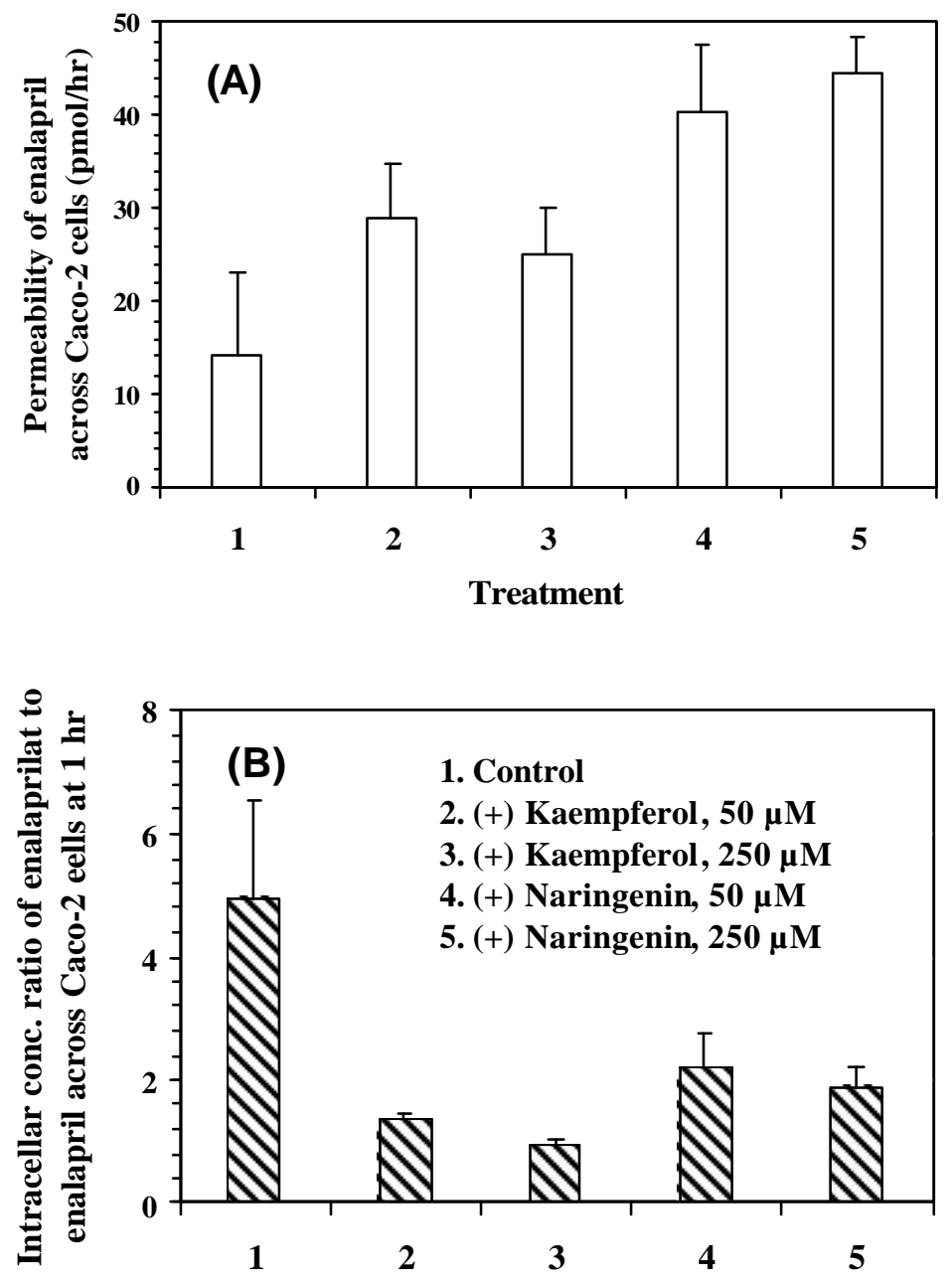

Figure 38. Effect of grapefruit juice constituents on $(A)$ enalapril $A \rightarrow B$ permeability across Caco-2 membrane (B) ratio of enalaprilat and enalapril in Caco-2 cells at $1 \mathrm{hr}$ 



Figure 39. Plasma concentration-time profiles of enalaprilat following oral administration of enalapril at $10 \mathrm{mg} / \mathrm{kg}$ with (A) water or kaempferol (2 and $10 \mathrm{mg} / \mathrm{kg}$ ) (B) water or naringenin (2 and $10 \mathrm{mg} / \mathrm{kg}$ ) 

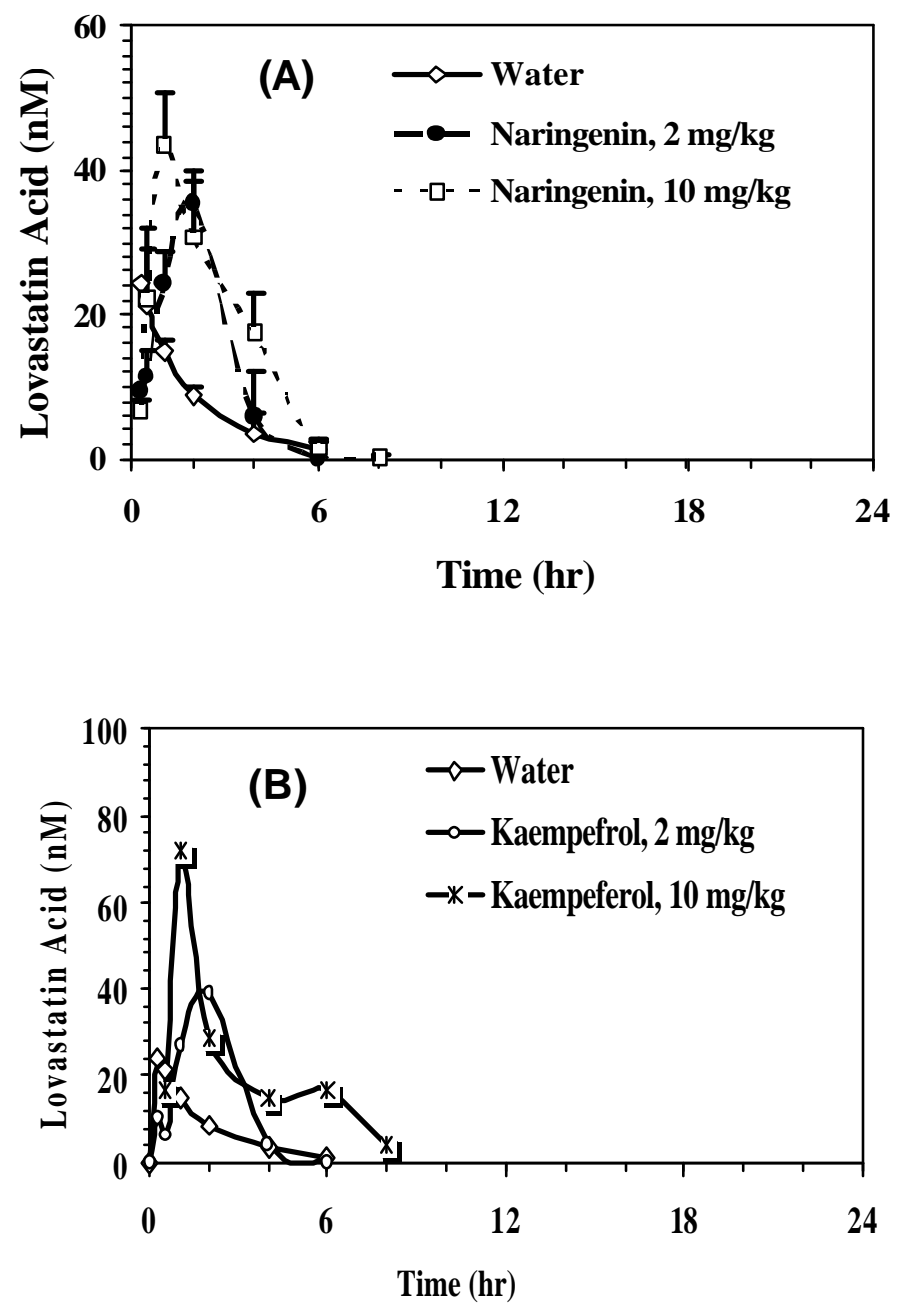

Figure 40. Plasma concentration-time profiles of lovastatin acid following oral administration of lovastatin at $10 \mathrm{mg} / \mathrm{kg}$ with $(A)$ water or naringenin (2 and $10 \mathrm{mg} / \mathrm{kg}$ ) (B) water or kaempferol (2 and $10 \mathrm{mg} / \mathrm{kg}$ ) 

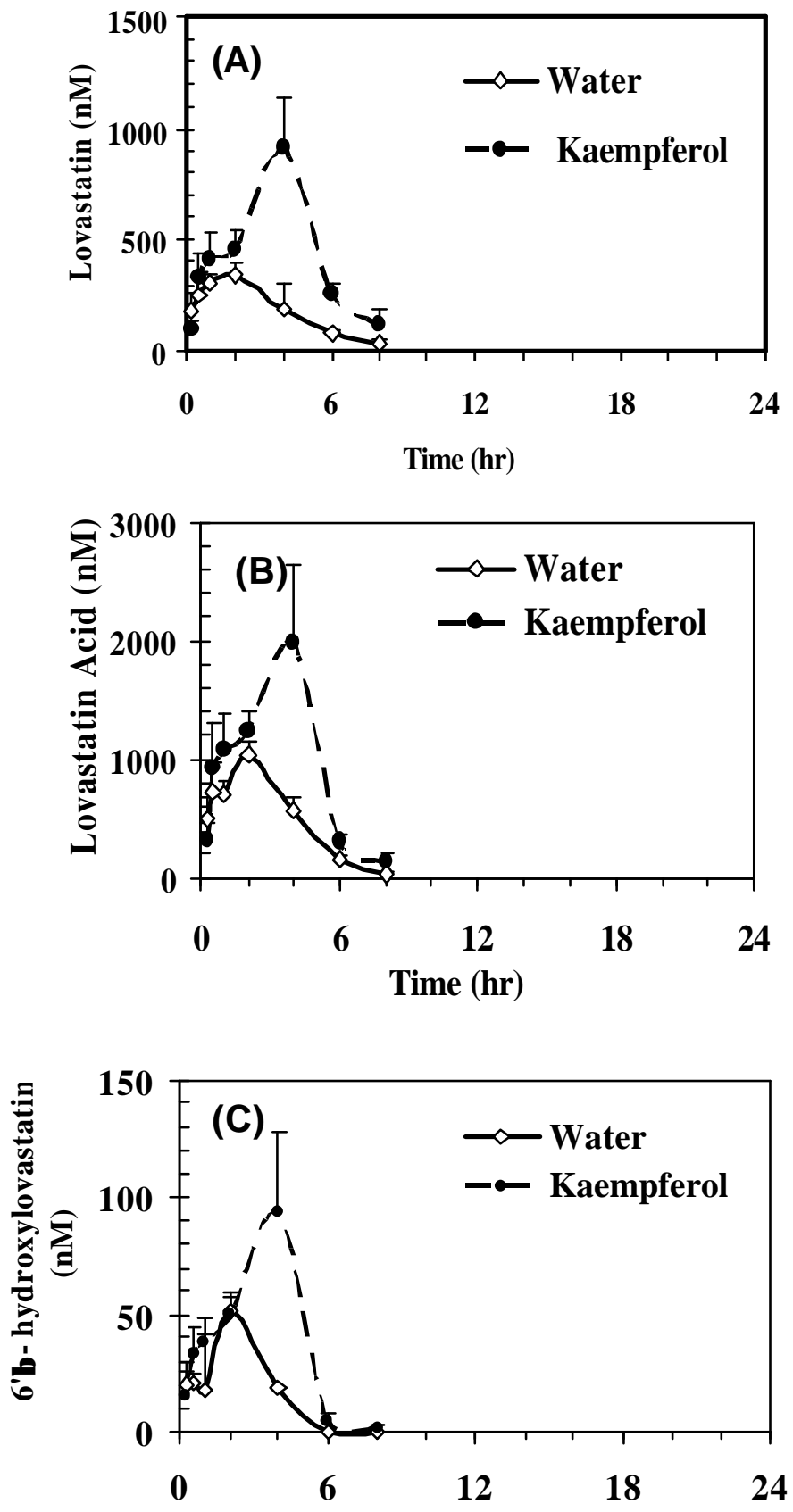

Time (hr)

Figure 41. Portal plasma concentration-time profiles of (A) lovastatin acid (B) lovastatin, and (C) 6' $\lesssim$-hydroxylovastatin following oral administration of lovastatin $(10 \mathrm{mg} / \mathrm{kg})$ with water or kaempferol $(10 \mathrm{mg} / \mathrm{kg})$ to rats 

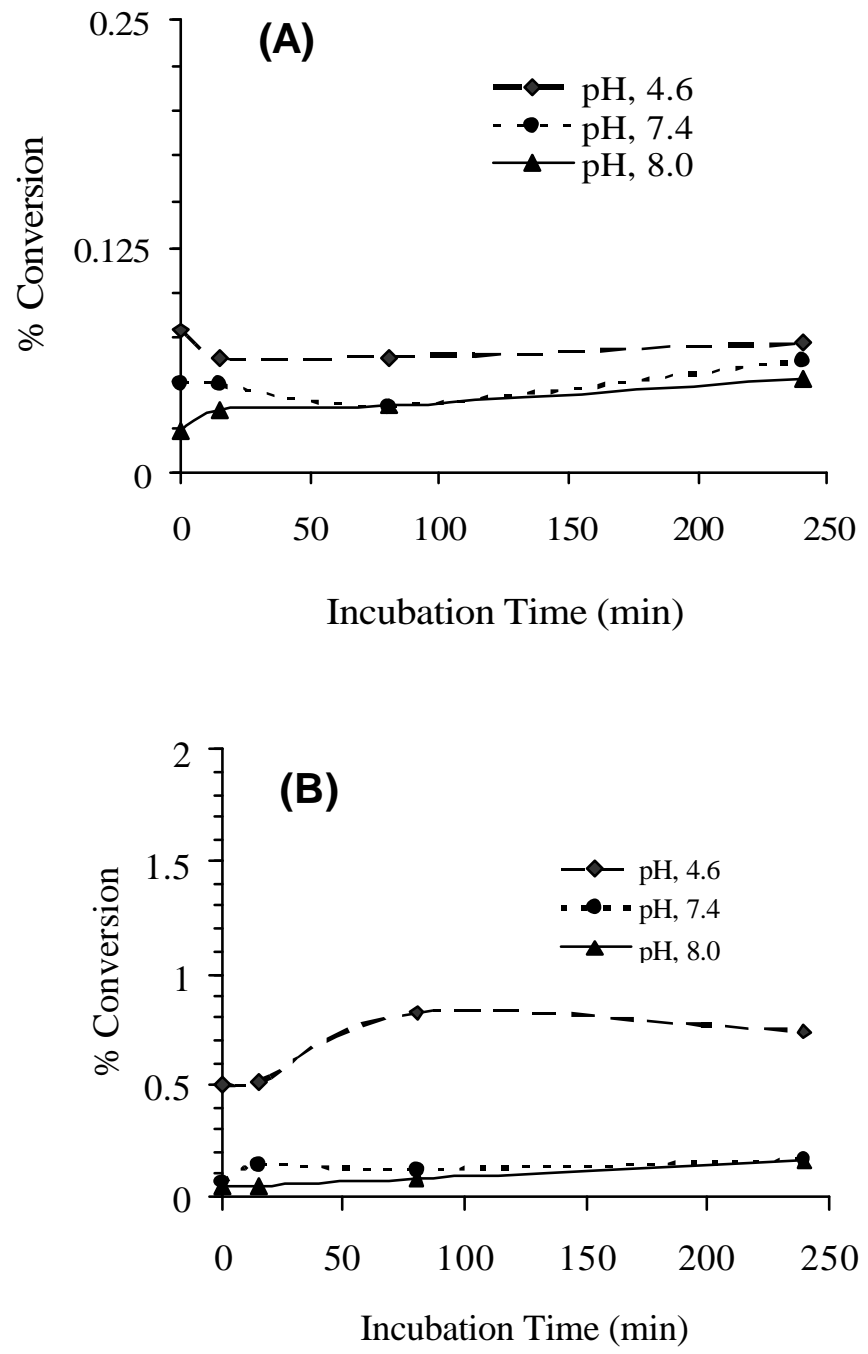

Figure 42. Chemical hydrolysis of (A) CPT11 (B) enalapril in $0.1 \mathrm{M}$ phosphate buffer, $\mathrm{pH}, 4.6,7.0$, and 8.0 


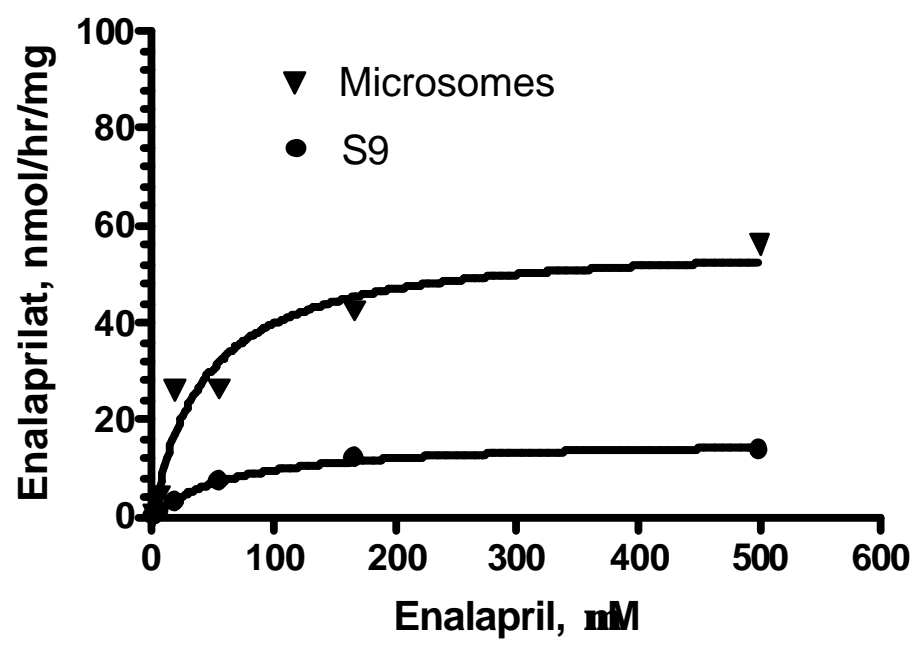

\begin{tabular}{ccc}
\hline & Microsomes & S9 \\
\hline Vmax, nmol/hr/mg & 57.2 & 16.2 \\
$\mathrm{Km}, \mathrm{nmol} / \mathrm{ml}$ & 43.6 & 68.3 \\
$\mathrm{Clint}, \mathrm{L} / \mathrm{hr} / \mathrm{kg}$ & 1.18 & 0.78 \\
$\mathrm{CL}, \mathrm{L} / \mathrm{hr} / \mathrm{kg}$ & 0.60 & 0.47 \\
\hline
\end{tabular}

Figure 43. Michaelis-Menten kinetics of CPT11hydrolysis in human liver microsomes and S9 fractions 


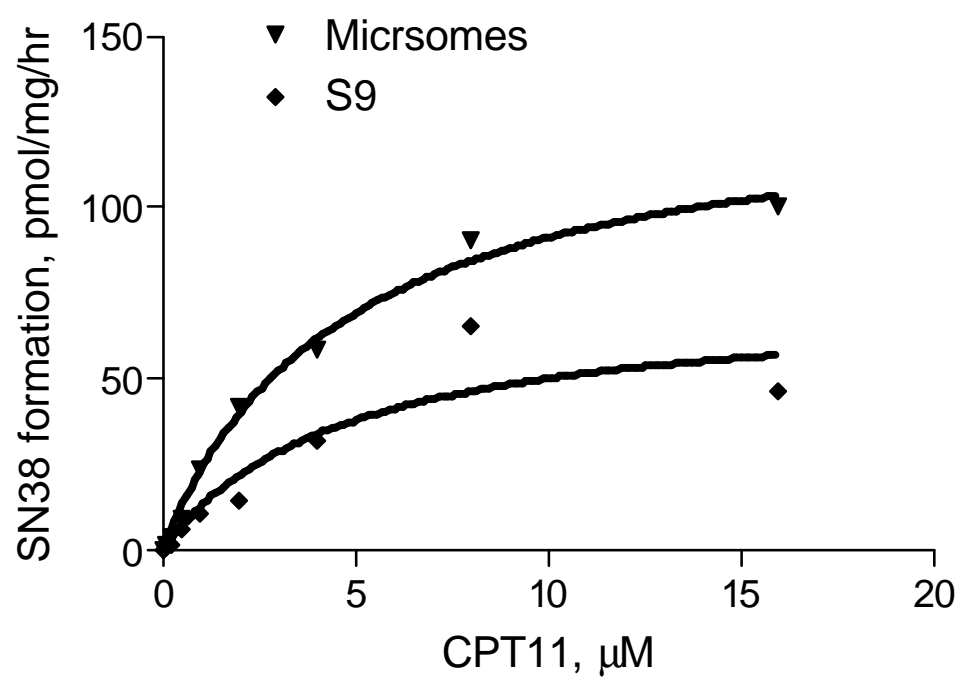

\begin{tabular}{ccc}
\hline & Microsomes & S9 \\
\hline Vmax nmol/hr/mg & 0.134 & 0.074 \\
$\mathrm{~km}, \mathrm{nmol} / \mathrm{ml}$ & 4.6 & 4.7 \\
Clint, L/hr/kg & 0.03 & 0.05 \\
$\mathrm{CL}, \mathrm{L} / \mathrm{hr} / \mathrm{kg}$ & 0.026 & 0.050 \\
\hline
\end{tabular}

Figure 44. Michaelis-Menten kinetics of enalapril hydrolysis in human liver microsomes and S9 fractions 
(A)

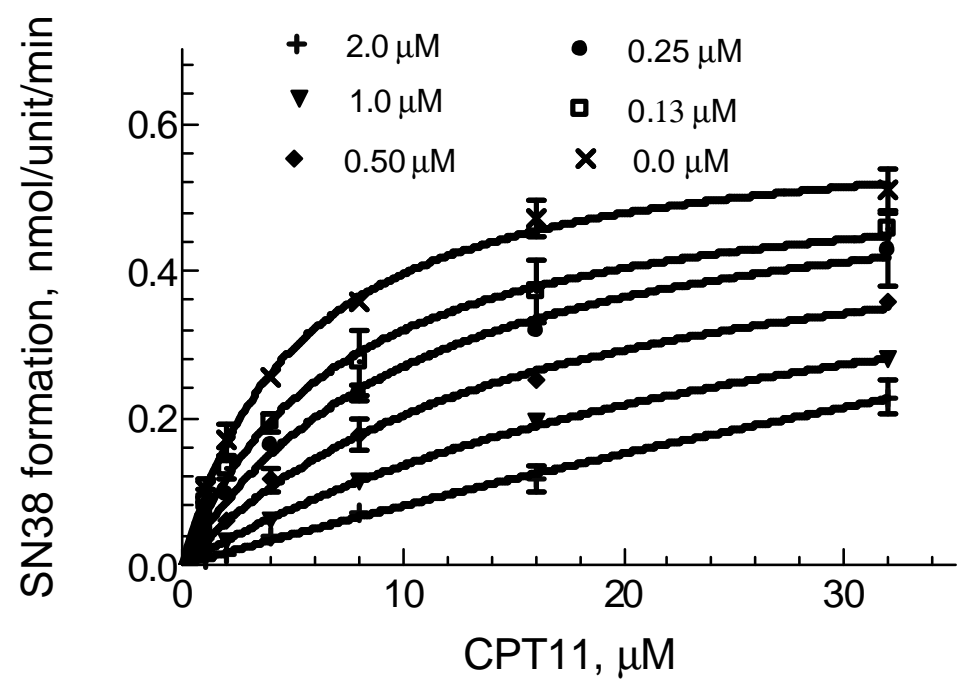

(B)

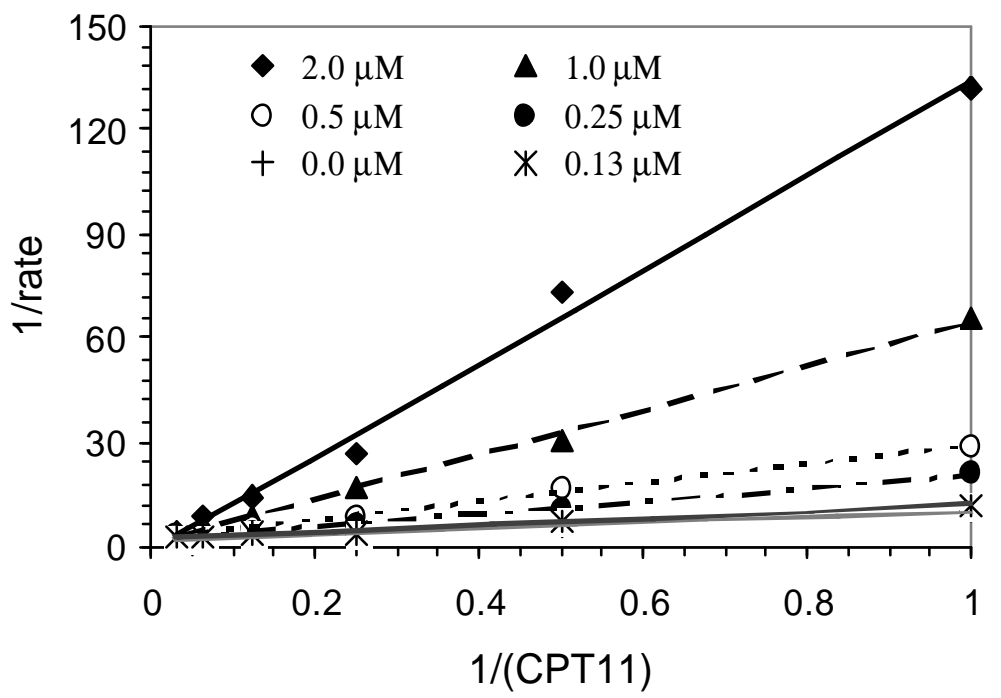

Figure 45. Michaelis-Menten kinetics of CPT11 hydrolysis by purified porcine esterases, and the effect of kaempferol (A) Direct plot (B) Primary Lineweaver-Burk plot 
(A)



(B)

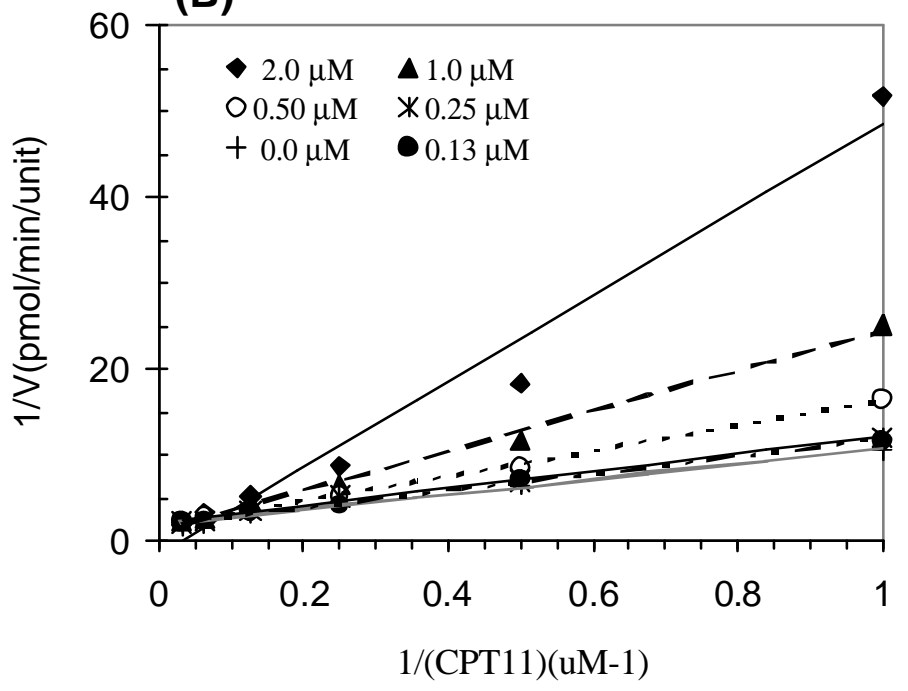

Figure 46. Michaelis-Menten kinetics of CPT11 hydrolysis by purified porcine esterases, and the effect of quercetin (A) Direct plot and (B) Primary Lineweaver-Burk plot 
(A)

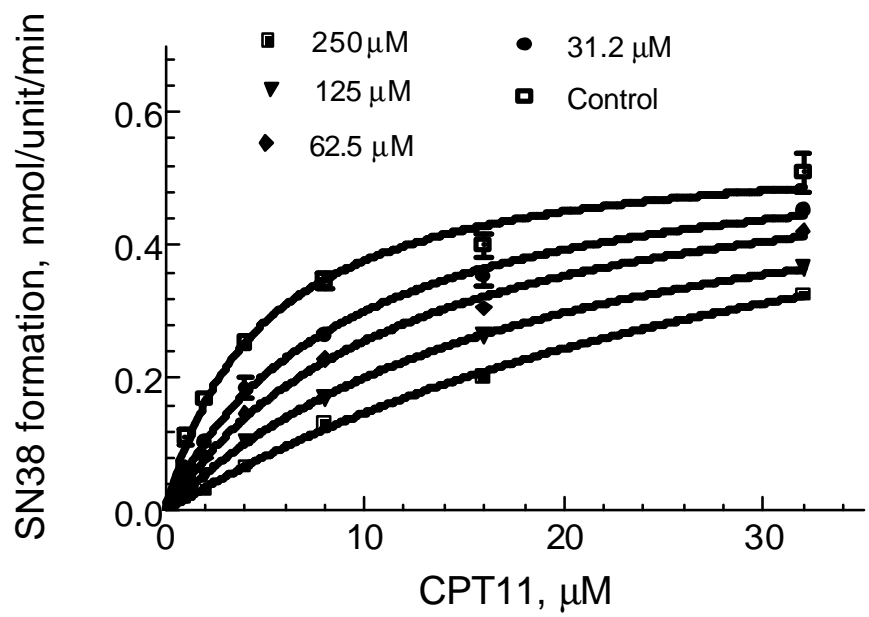

(B)

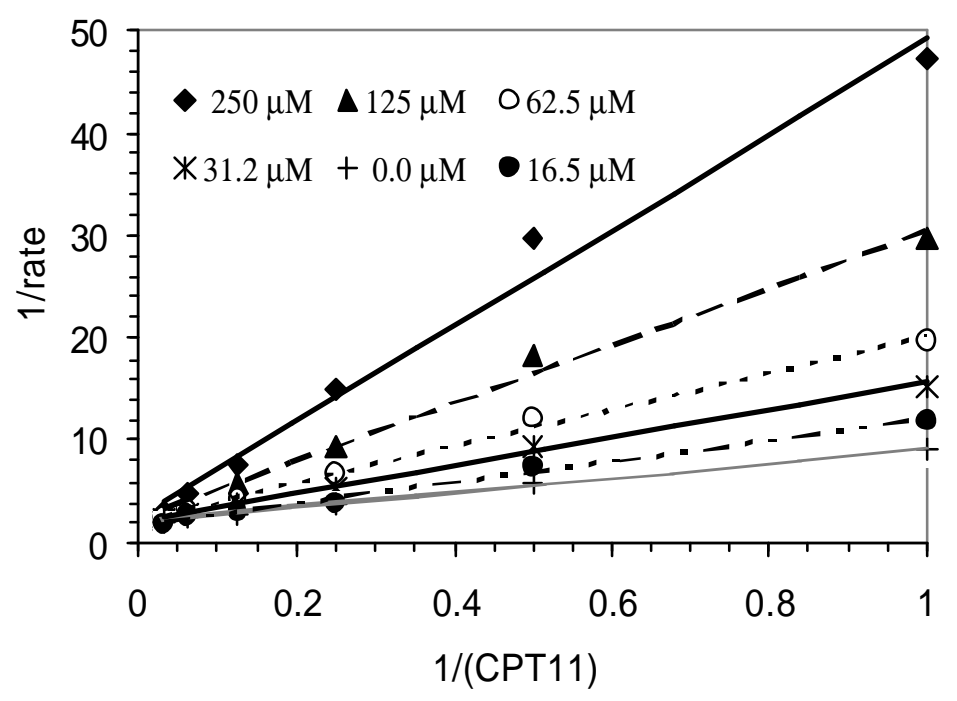

Figure 47. Michaelis-Menten kinetics of CPT11 hydrolysis by purified porcine esterases, and the effect of naringenin (A) Direct plot and (B) Primary Lineweaver-Burk plot 
(A)

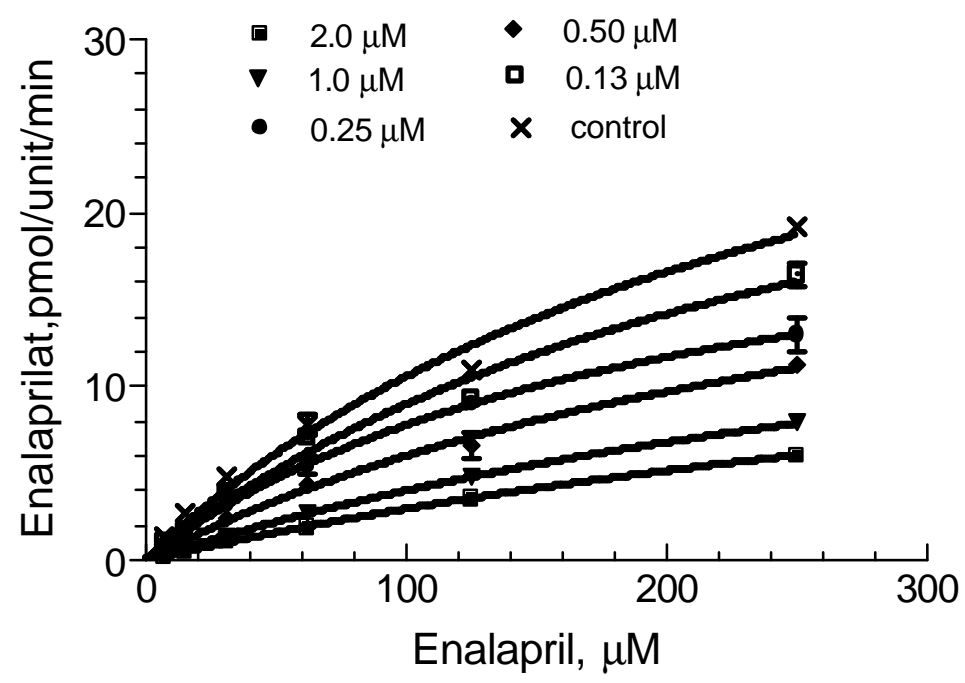

(B)

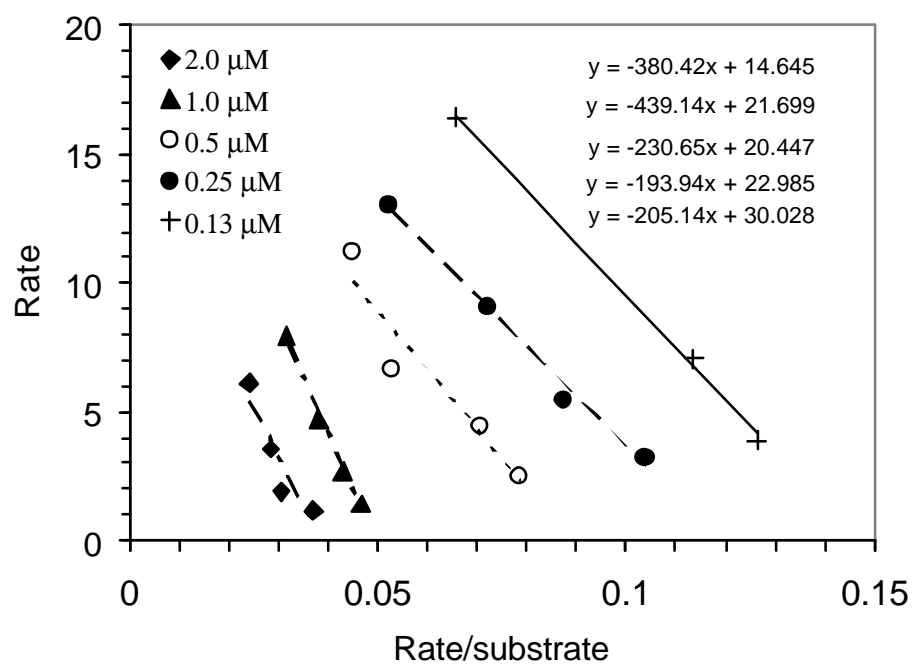

Figure 48. Michaelis-Menten kinetics of enalapril hydrolysis by purified porcine esterases, and the effect of kaempferol (A) Direct plot and (B) Eadie-Hofstee plot 
(A)

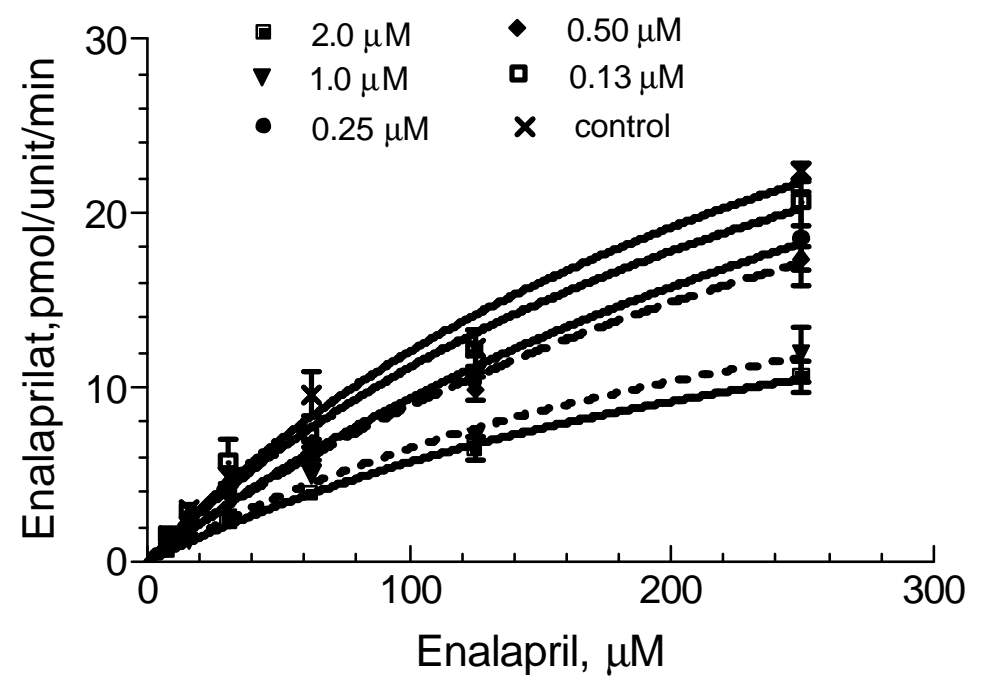

(B)



Figure 49. Michaelis-Menten kinetics of enalapril hydrolysis by purified porcine esterases, and the effect of quercetin (A) Direct plot and (B) Eadie-Hofstee plot 
(A)



(B)

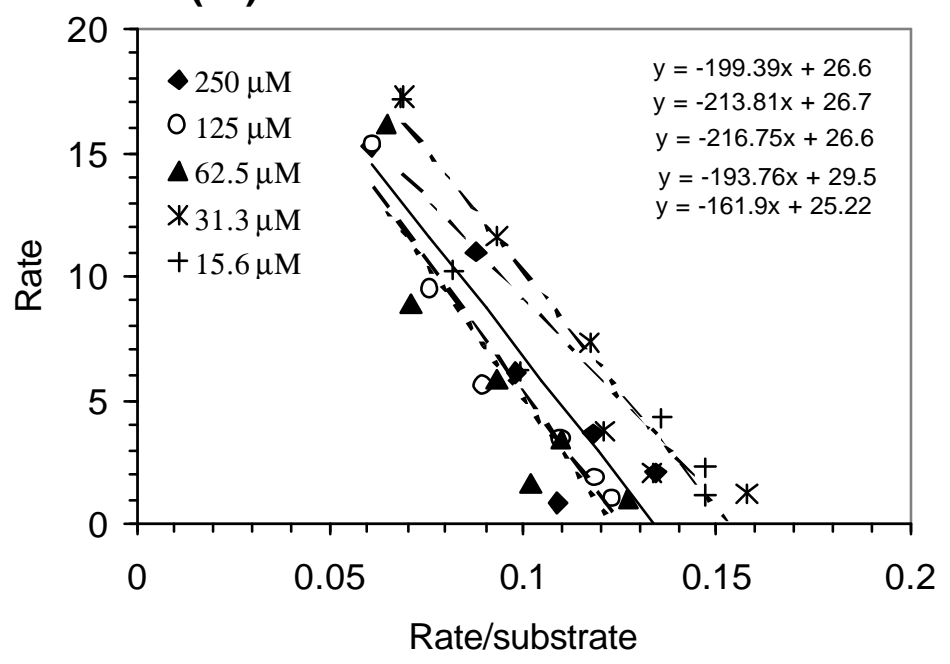

Figure 50. Michaelis-Menten kinetics of enalapril hydrolysis by purified porcine esterases, and the effect of naringenin (A) Direct plot and (B) Eadie-Hofstee plot 


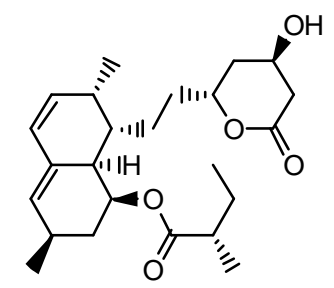

Lovastatin

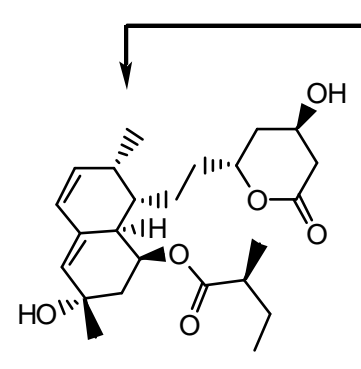

6' $\beta$-Hydroxylovastatin
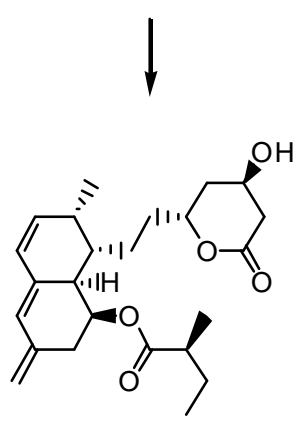

6'-Exomethylene

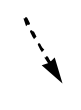

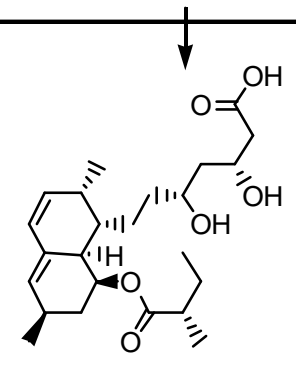

Lovastatin acid





3"-Hydroxylovastatin



3"-Hydroxylovastatin acid

Figure 51. Metabolism of lovastatin in human liver microsomes 
<smiles>CC(=O)Oc1ccc([N+](=O)[O-])cc1</smiles>

Para-nitropheny lacetate<smiles>CCOC(=O)[C@H](CCc1ccccc1)N[C@@H](C)C(=O)N1CCC[C@H]1C(=O)O</smiles>

Enalapril

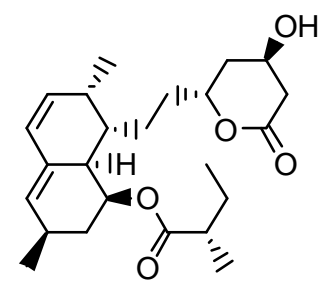

Lovastatin<smiles>CCc1c2c(nc3ccc(OC(=O)N4CCC(N5CCCCC5)CC4)cc13)Cn1c-2cc2c(c1=O)COC(=O)[C@H]2O</smiles>

CPT11
Esterase

Esterase

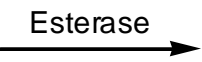

Esterase<smiles>O=[N+]([O-])c1ccc(O)cc1</smiles>

Para-nitrophenol<smiles>C[C@H](N[C@@H](CCc1ccccc1)C(=O)O)C(=O)N1CCC[C@H]1C(=O)O</smiles>

Enalaprilat



Lovastatin acid<smiles>CCc1c2c(nc3ccc(O)cc13)-c1cc3c(c(=O)n1C2)COC(=O)[C@@]3(O)CC</smiles>

SN-38

Figure 52. Structures of studied carboxyl esters and corresponding free acids 


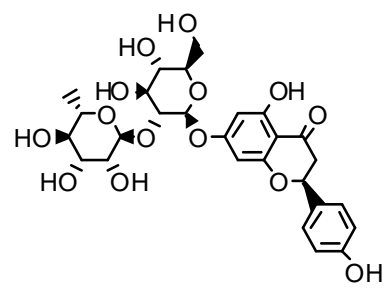

Naringin<smiles>O=c1c(O)c(-c2ccc(O)cc2O)oc2cc(O)cc(O)c12</smiles>

Morin<smiles>O=c1c(O)c(-c2ccccc2)oc2cc(O)cc(O)c12</smiles>

Galangin<smiles>O=c1c(O)c(-c2ccc(O)c(O)c2)oc2cc(O)cc(O)c12</smiles>

Quercetin

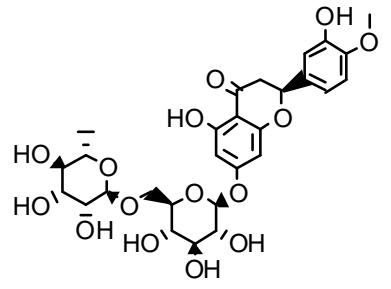

Hesperidin

Naringenin

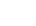


<smiles>O=S(=O)(F)Cc1ccccc1</smiles>

phenylmethanesulfonyl fluoride

(PMSF)<smiles>CN(CC(=O)O)C(=O)CN</smiles>

Glycine, N-glycyl-N-methyl

(Gly-Sar)<smiles>O=[N+]([O-])c1ccc(OP(=O)(O[Na])Oc2ccc([N+](=O)[O-])cc2)cc1</smiles>

Phosphoric acid, bis(4-nitrophenyl) ester

(BNPP)

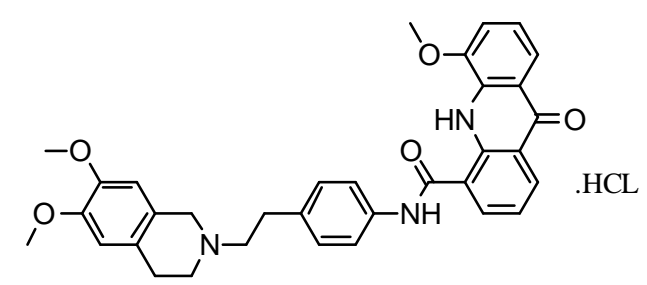

9,10-dihydro-5-methoxy-9-oxo-N-[4-[2-(1,2,3,4tetrahydro-6,7-dimethoxy-2-isoquinolinyl)ethyl ]phenyl]-4-acridine-carboxamide hydrochloride

(GF120918)

Figure 54. Structures of PMSF, BNPP, Gly-Sar, and GF120918 